\title{
Hipersuperfícies em espaços produto com curvaturas principais constantes
}

\author{
Eliane da Silva dos Santos
}

\author{
TESE APRESENTADA \\ $\mathrm{AO}$ \\ Instituto de MAtemática e EstatísticA \\ DA \\ UNIVERSIDADE DE SÃO PAULO \\ PARA \\ OBTENÇÃO DO TÍTULO \\ $\mathrm{DE}$ \\ DOUTORA EM CIÊNCIAS
}

\section{Programa: Matemática \\ Orientadora: Profa. Dra. Rosa Maria dos Santos Barreiro Chaves}

Durante o desenvolvimento deste trabalho a autora recebeu auxílio financeiro da CAPES e $\mathrm{CNPq}$

São Paulo, novembro de 2013 



\section{Hipersuperfícies em espaços produto com curvaturas principais constantes}

Este exemplar corresponde à redação final da tese devidamente corrigida

e defendida por Eliane da Silva dos Santos

e aprovada pela Comissão Julgadora.

Banca Examinadora:

- Profa. Dra. Rosa Maria Barreiro Chaves (orientadora) - IME-USP.

- Prof. Dr. Antônio Carlos Asperti - IME-USP.

- Prof. Dr. Luíz Amâncio Machado Souza - UNIRIO.

- Profa. Dra. Irene Ignazia Onnis - ICMC-USP.

- Prof. Dr. Márcio Fabiano da Silva - UFABC. 

Deus não escolhe os capacitados, capacita os escolhidos. Fazer ou não fazer algo só depende de nossa vontade e perseverança. Albert Einsten 

Aos meus pais Lindinalva e Antônio Carlos, às minhas irmãs Elane e Elma, ao meu sobrinho Gabriel e ao meu companheiro Oscar. 



\section{Agradecimentos}

Agradeço primeiramente à Deus, pelo dom da vida e por ter me capacitado para realizar este trabalho.

À minha orientadora Rosa Chaves, uma das pessoas mais belas que já conheci, muito obrigada pela paciência, atenção e dedicação. É sempre um grande prazer trabalhar com você. Agradeço também aos professores Asperti, Plínio, Marcos Alexandrino, Henri, Ruy e Amâncio pelas valiosas sugestões. Ao pessoal do seminário de geometria, Bárbara, Alexandre, José Vilhena e Juan Fernando. Aos funcionários e professores do IME.

O desenvolvimento desta tese foi apoiado pelas agências CAPES e CNPq. O suporte dessas agências foram fundamentais para a realização desse trabalho.

À minha família, em especial aos meus pais, pelo amor incondicional, às minhas irmãs Elane e Elma, pelo apoio, pela força e orações. Ao meu sobrinho Gabriel, por encher meu coração de alegria e me fortalecer. Aos meus avós e meus sogros Nubia e Carlos, pelas orações. À tia Lina, pela atenção e amor, e ao meu cunhado Gilmar pelo apoio na minha chegada a São Paulo.

Aproveito para agradecer às minhas amigas Rose e Manu, pelas discussões matemáticas, pelo apoio, por me animar nos momentos difíceis e pela alegria contagiante de vocês. Ao meu amigo Maikel, por me ouvir e apoiar em momentos difíceis. Aos meus amigos Paião, Gláucia, Vinícius, Glauce, Tatá, Adilson e Anderson pela companhia diária na sala de estudo, pelas conversas, pela amizade, por tornar meus dias mais agradáveis.

Ao meu grande companheiro Oscar, que está ao meu lado em todos os momentos, me dando força nos momentos difíceis, comemorando comigo cada vitória, me apoiando e fortalecendo. Muito obrigada pelo seu carinho e pelo seu amor.

Não podia deixar de agradecer aos meus professores da UFBA, em especial Enaldo, José Fernandes e José Nelson por contribuirem para a minha formação matemática e crescimento

acadêmico. Às minhas amigas Vanessa e Fabiana pela amizade e apoio, desde a época da graduação.

A todos que de alguma forma colaboraram para o desenvolvimento deste trabalho, muito obrigada!!! 


\section{Resumo}

Santos, E. S. Hipersuperfícies em espaços produto com curvaturas principais constantes. 2013. Tese (Doutorado) - Instituto de Matemática e Estatística, Universidade de São Paulo, São Paulo, 2013.

Neste trabalho, classificamos localmente as hipersuperfícies dos espaços produto $\mathbb{S}^{n} \times \mathbb{R}$ e $\mathbb{H}^{n} \times \mathbb{R}, n \neq 3$, com $g$ curvaturas principais constantes e distintas, $g \in\{1,2,3\}$. Verificamos que tais hipersuperfícies são isoparamétricas de $\mathbb{Q}_{c}^{n} \times \mathbb{R}$. Além disso, encontramos uma condição necessária e suficiente para que uma hipersuperfície isoparamétrica de $\mathbb{Q}_{c}^{n} \times \mathbb{R}$ que possui fibrado normal plano, quando observada como uma subvariedade de codimensão dois de $\mathbb{R}^{n+2}$ contendo $\mathbb{S}^{n} \times \mathbb{R}$ e de $\mathbb{L}^{n+2}$ contendo $\mathbb{H}^{n} \times \mathbb{R}$, tenha curvaturas principais constantes.

Palavras chave: hipersuperfícies em espaços produto; curvaturas principais constantes; hipersuperfícies isoparamétricas. 


\section{Abstract}

Santos, E. S. Hypersurfaces in product spaces with constant principal curvatures. 2013. Tese (Doutorado) - Instituto de Matemática e Estatística, Universidade de São Paulo, São Paulo, 2013.

In this work, we classify locally the hypersurfaces in product spaces $\mathbb{S}^{n} \times \mathbb{R}$ and $\mathbb{H}^{n} \times \mathbb{R}$, $n \neq 3$, with $g$ distinct constant principal curvatures, $g \in\{1,2,3\}$. We verify that such hypersurfaces are isoparametric in $\mathbb{Q}_{c}^{n} \times \mathbb{R}$. Furthermore, we find a necessary and sufficient condition for an isoparametric hypersurface in $\mathbb{Q}_{c}^{n} \times \mathbb{R}$ with flat normal bundle, when regarded as a submanifold with codimension two of the flat spaces $\mathbb{R}^{n+2}$ containing $\mathbb{S}^{n} \times \mathbb{R}$ and $\mathbb{L}^{n+2}$ containing $\mathbb{H}^{n} \times \mathbb{R}$, having constant principal curvatures.

Keywords: hypersurfaces in product spaces; constant principal curvatures; isoparametric hypersurfaces. 


\section{Sumário}

Introdução $\quad$ xiii

1 Preliminares 1

1.1 Algumas classes especiais de hipersuperfícies em $\mathbb{Q}_{c}^{n} \times \mathbb{R} \ldots \ldots 1$

1.1.1 Hipersuperfícies totalmente geodésicas . . . . . . . . . . . . . 4

1.1 .2 Hipersuperfícies rotacionais . . . . . . . . . . . . . 4

1.1.3 Hipersuperfícies que possuem o campo $T$ como uma direção principal 10

1.2 Hipersuperfícies isoparamétricas em $\mathbb{Q}_{c}^{n} \ldots \ldots \ldots \ldots \ldots . \ldots \ldots$

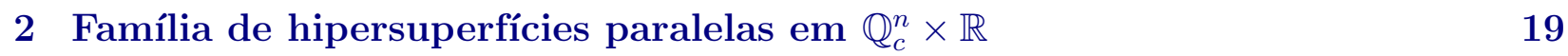

2.1 Existência de um referencial local de direções principais . . . . . . . . . . . 19

2.2 Construção da família de paralelas . . . . . . . . . . . . . . . . . . 21

2.3 Relação entre as curvaturas principais de uma hipersuperfície e sua família de paralelas . . . . . . . . . . . . . . . . . . . . . 24

3 Hipersuperfícies com curvaturas principais constantes em $\mathbb{Q}_{c}^{n} \times \mathbb{R} \quad 31$

3.1 Hipersuperfícies com curvaturas principais constantes que possuem o campo T como uma direção principal . . . . . . . . . . . . . . . . . . . . . . 31

3.2 Multiplicidade das curvaturas principais . . . . . . . . . . . . . . 37

3.3 Hipersuperfícies de $\mathbb{Q}_{c}^{n} \times \mathbb{R}$ com curvaturas principais constantes para $g \in\{1,2,3\} \quad 40$

$\begin{array}{ll}\text { Referências Bibliográficas } & 55\end{array}$ 


\section{Introdução}

O estudo de hipersuperfícies em espaços produto tem atraído a atenção de vários geômetras nos últimos anos. A seguir, faremos um breve histórico desse estudo.

Inicialmente foram estudadas as superfícies de curvatura média constante e, em particular, as superfícies mínimas, nos trabalhos de H. Rosenberg, W. Meeks e U. Abresch em [2], [20] e [29]. Elas também foram abordadas por I. Onnis em [22], [23], [28] e B. Nelli em [26], entre outros.

Em [3] e [4], J. Aledo, J. Espinar e J. Gálvez descreveram as superfícies de $\mathbb{S}^{2} \times \mathbb{R}$ e $\mathbb{H}^{2} \times \mathbb{R}$ com curvatura Gaussiana constante. Em particular, eles mostraram que as superfícies completas de curvatura Gaussiana constante $K(I)>0$ em $\mathbb{H}^{2} \times \mathbb{R}$ (respectivamente, $K(I)>1$ em $\left.\mathbb{S}^{2} \times \mathbb{R}\right)$ são rotacionais. Eles provaram também a não existência de superfícies completas com curvatura Gaussiana constante $K(I)<-1$ em $\mathbb{H}^{2} \times \mathbb{R}$ (respectivamente $K(I)<-1$ e $0<K(I)<1 \mathrm{em} \mathbb{S}^{2} \times \mathbb{R}$ ). Além disso, J. Espinar e J. Gálvez, juntamente com H. Rosenberg, mostraram em [17] que uma superfície completa com curvatura extrínseca constante positiva em $\mathbb{S}^{2} \times \mathbb{R}$ e $\mathbb{H}^{2} \times \mathbb{R}$ é uma esfera rotacional.

Posteriormente também despertaram interesse as hipersuperfícies, $n$-dimensionais, em $\mathbb{S}^{n} \times \mathbb{R}$ e $\mathbb{H}^{n} \times \mathbb{R}$. Para unificar a linguagem, denotaremos por $\mathbb{Q}_{c}^{n}$ a esfera $\mathbb{S}^{n}$, se $c=1$, ou o espaço hiperbólico $\mathbb{H}^{n}$, se $c=-1$.

As hipersuperfícies rotacionais em $\mathbb{Q}_{c}^{n} \times \mathbb{R}$ foram parametrizadas por F. Dillen, J. Fastenakels e J. Van Der Veken em [15] como uma extensão do trabalho [16], de M.P. do Carmo e M. Dajczer, sobre rotacionais em espaços forma.

As hipersuperfícies em $\mathbb{Q}_{c}^{n} \times \mathbb{R}$ que possuem um campo especial $T$ como direção principal foram classificadas localmente por R. Tojeiro em [32]. O campo diferenciável $T$ e a função diferenciável $\nu$ são definidos por

$$
\partial / \partial t=d f(T)+\nu \eta
$$

onde $f$ é uma imersão de uma variedade Riemanniana $n$-dimensional $M^{n}$ em $\mathbb{Q}_{c}^{n} \times \mathbb{R}$, com campo normal unitário $\eta$ e $\partial / \partial t$ é um campo unitário tangente ao segundo fator $\mathbb{R}$. Em 
particular, foram classificadas as hipersuperfícies de ângulo constante, isto é, as que têm a função $\nu$ constante.

Em [19], R. Tojeiro e F. Manfio classificaram localmente as hipersuperfícies em $\mathbb{Q}_{c}^{n} \times \mathbb{R}$, $n \geq 3$, com curvatura seccional constante. Eles mostraram que para $n \geq 4$ existe hipersuperfície de curvatura seccional constante $k$ em $\mathbb{S}^{n} \times \mathbb{R}$ (respectivamente, $\mathbb{H}^{n} \times \mathbb{R}$ ), mesmo localmente, se $k>1$ (respectivamente, se $k \geq-1$ ), e para quaisquer tais valores de $k$ ela deve ser um aberto de uma hipersuperfície completa de rotação. No caso $n=3$, aparece exatamente uma classe de hipersuperfícies com curvatura seccional constante, não rotacionais, de $\mathbb{Q}_{c}^{n} \times \mathbb{R}$. Cada elemento desta classe em $\mathbb{S}^{3} \times \mathbb{R}$ (respectivamente, $\mathbb{H}^{3} \times \mathbb{R}$ ) tem curvatura seccional constante $k \in(0,1)$ (respectivamente, $k \in(-1,0)$ ) e possui ângulo constante.

Tendo em vista as classificações mencionadas acima, surgiu, naturalmente, o interesse em pesquisar quais são as hipersuperfícies de $\mathbb{Q}_{c}^{n} \times \mathbb{R}$ que possuem curvaturas principais constantes e responder se elas são isoparamétricas em $\mathbb{Q}_{c}^{n} \times \mathbb{R}$.

É bem conhecido que uma hipersuperfície de um espaço forma é isoparamétrica se, e somente se, suas curvaturas principais são constantes, porém isso não ocorre em ambientes mais gerais. A segunda questão do parágrafo anterior foi motivada pelo fato de que não é verdade que hipersuperfícies isoparamétricas de qualquer variedade Riemanniana possuem curvaturas principais constantes. Em [34] podem ser encontrados exemplos de hipersuperfícies isoparamétricas em espaços projetivos complexos que não possuem curvaturas principais constantes. Para mais detalhes indicamos o trabalho [31] de G. Thorbergsson.

As hipersuperfícies isoparamétricas de $\mathbb{R}^{n}$ e $\mathbb{H}^{n}$ podem ter no máximo duas curvaturas principais distintas. Já as isoparamétricas de $\mathbb{S}^{n}$ podem ter 1, 2, 3, 4 ou 6 curvaturas principais distintas. Cartan mostrou em uma série de quatro artigos [5], [6], [7] e [8], publicados no período de 1938 a 1940, que a teoria de hipersuperfícies isoparamétricas na esfera é mais complicada. Ele classificou essas hipersuperfícies com $g \leq 3$, onde $g$ é o número de curvaturas principais distintas. O caso $g=4$ e $g=6$ tem despertado interesse de muitos pesquisadores da área. Abordaremos esse assunto com mais detalhes na Seção 2 do Capítulo 1.

Pesquisando quais são as hipersuperfícies em $\mathbb{Q}_{c}^{n} \times \mathbb{R}$ que possuem curvaturas principais constantes e buscando responder se elas são isoparamétricas de $\mathbb{Q}_{c}^{n} \times \mathbb{R}$, classificamos localmente as hipersuperfícies em $\mathbb{Q}_{c}^{n} \times \mathbb{R}, n \neq 3$, com até 3 curvaturas principais constantes e distintas. Mais precisamente, mostramos o seguinte resultado, que será apresentado no Capítulo 3.

Teorema 1 (Teorema 3.3.4). Seja $f: M^{n} \rightarrow \mathbb{Q}_{c}^{n} \times \mathbb{R}$ uma hipersuperfície com curvaturas principais constantes.

(i) Se $g=1$ e $n \geq 2$ então $f\left(M^{n}\right)$ é um subconjunto aberto de $\mathbb{Q}_{c}^{n} \times\left\{t_{0}\right\}$, para algum $t_{0} \in \mathbb{R}$, ou de um produto Riemanniano $M^{n-1} \times \mathbb{R}$. Nesse último caso, para $c=1, M^{n-1}$ é uma 
esfera totalmente geodésica em $\mathbb{S}^{n}$ e, para $c=-1, M^{n-1}$ é um hiperplano totalmente geodésico em $\mathbb{H}^{n}$.

(ii) Se $g=2$ e $n \geq 2$ então $c=-1$ e $f$ é localmente dada por $f(x, s)=h_{s}(x)+B s \partial / \partial t$, para algum $B \in \mathbb{R}, B>0$, com $M^{n}=M^{n-1} \times I$, onde $h_{s}$ é uma família de horosferas em $\mathbb{H}^{n}$, ou $f\left(M^{n}\right)$ é um subconjunto aberto de um produto Riemanniano $M^{n-1} \times \mathbb{R}$. Nesse último caso, se c $=1$ então $M^{n-1}$ é uma esfera não totalmente geodésica em $\mathbb{S}^{n}$ e, se $c=-1, M^{n-1}$ é uma hipersuperfície equidistante, uma horosfera ou uma hiperesfera em $\mathbb{H}^{n}$.

(iii) Se $g=3, n \geq 4$ e as multiplicidades das curvaturas principais são constantes, então para $c=1, f\left(M^{n}\right)$ é um subconjunto aberto de um produto Riemanniano $\mathbb{S}^{p}(r) \times \mathbb{S}^{q}(s) \times \mathbb{R}$, com $n=p+q+1$ e $r^{2}+s^{2}=1$, ou do produto $M^{n-1} \times \mathbb{R}$, onde $M^{n-1}$ é uma hipersuperfície de Cartan para $n \in\{4,7,13,25\}$. Se $c=-1, f\left(M^{n}\right)$ é um aberto do produto Riemanniano $\mathbb{S}^{k} \times \mathbb{H}^{n-k-1} \times \mathbb{R}$.

Além disso, obtivemos, no resultado que segue, a condição necessária e suficiente para que uma hipersuperfície isoparamétrica em $\mathbb{Q}_{c}^{n} \times \mathbb{R}$, que possui $T$ como uma direção principal, tenha curvaturas principais constantes.

Teorema 2 (Teorema 2.3.5). Seja $f: M^{n} \rightarrow \mathbb{Q}_{c}^{n} \times \mathbb{R}$ uma hipersuperfície isoparamétrica que possui $T$ como direção principal. Então $f$ possui curvaturas principais constantes se, $e$ somente se, $\|T\|$ é constante.

Concluimos que as hipersuperfícies, classificadas pelo Teorema 3.3.4, possuem $\|T\|$ constante e portanto são isoparamétricas em $\mathbb{Q}_{c}^{n} \times \mathbb{R}$.

Este trabalho está dividido em três capítulos, sendo que o primeiro contém alguns preliminares e os dois restantes são formados pelos resultados originais obtidos nesta tese. A seguir, daremos uma breve descrição sobre cada um deles.

O Capítulo 1 é constituído pelos resultados estabelecidos na literatura que são necessários para o desenvolvimento da tese. Iniciamos este capítulo expondo algumas propriedades básicas de hipersuperfícies em $\mathbb{Q}_{c}^{n} \times \mathbb{R}$ e apresentando resultados relacionados a algumas classes especiais, tais como as totalmente geodésicas, as rotacionais e as que possuem o campo $T$ como direção principal. Posteriormente apresentamos alguns resultados de hipersuperfícies isoparamétricas em $\mathbb{Q}_{c}^{n}$.

No Capítulo 2, apresentamos um resultado que garante a existência de um referencial de direções principais ortogonais em hipersuperfícies que possuem curvaturas principais com multiplicidades constantes. Esse resultado é bastante importante pois utilizamos esse referencial no decorrer do trabalho. Construimos uma família de hipersuperfícies paralelas a uma 
hipersuperfície que possui o campo $T$ como direção principal e relacionamos as suas respectivas curvaturas principais. Em seguida, apresentamos sob quais condições uma hipersuperfície isoparamétrica em $\mathbb{Q}_{c}^{n} \times \mathbb{R}$, que tem $T$ como direção principal, possui curvaturas principais constantes.

Iniciamos o Capítulo 3 fazendo um estudo sobre as hipersuperfícies em $\mathbb{Q}_{c}^{n} \times \mathbb{R}$ que possuem curvaturas principais constantes, na classe das que têm o campo $T$ como direção principal. Em seguida, estudamos as hipersuperfícies que possuem curvaturas principais constantes com suas respectivas multiplicidades constantes, sem hipóteses adicionais. Apresentamos alguns resultados relacionados às multiplicidades de tais curvaturas e, finalmente, o teorema principal desta tese que classifica localmente tais hipersuperfícies com até três curvaturas principais distintas. 


\section{Capítulo 1}

\section{Preliminares}

Este capítulo contém resultados já estabelecidos na literatura que serão utilizados nos capítulos posteriores desta tese. Apresentamos propriedades e conceitos básicos fundamentais de hipersuperfícies em $\mathbb{Q}_{c}^{n} \times \mathbb{R}$. Dentre algumas classes especiais dessas hipersuperfícies, destacamos aqui as que possuem fibrado normal plano quando consideradas como subvariedades de codimensão $2 \mathrm{em} \mathbb{E}^{n+2}$, as rotacionais, as totalmente geodésicas e as umbílicas. Além disso, apresentamos alguns resultados de hipersuperfícies isoparamétricas em $\mathbb{Q}_{c}^{n}$.

\subsection{Algumas classes especiais de hipersuperfícies em $\mathbb{Q}_{c}^{n} \times \mathbb{R}$}

Denotamos por $\mathbb{E}^{n+2}$ o espaço Euclidiano $\mathbb{R}^{n+2}$ ou o espaço Lorentziano $\mathbb{L}^{n+2}$, de acordo com $c=1$ ou $c=-1$, respectivamente. Consideramos

$$
\begin{gathered}
\mathbb{E}^{n+1}=\left\{\left(x_{1}, \ldots, x_{n+2}\right) \in \mathbb{E}^{n+2} / x_{n+2}=0\right\} \quad \mathrm{e} \\
\mathbb{Q}_{c}^{n}=\left\{\left(x_{1}, \ldots, x_{n+1}\right) \in \mathbb{E}^{n+1} / c x_{1}^{2}+x_{2}^{2}+\cdots+x_{n+1}^{2}=c\right\}
\end{gathered}
$$

com $x_{1}>0$, se $c=-1$. Ou seja, denotamos por $\mathbb{Q}_{c}^{n}$, a esfera $\mathbb{S}^{n}$, se $c=1$, ou o espaço hiperbólico $\mathbb{H}^{n}$, se $c=-1$. Dada uma imersão isométrica $f: M^{n} \rightarrow \mathbb{Q}_{c}^{n} \times \mathbb{R}$, seja $\eta$ o campo normal unitário e $\partial / \partial t$ o campo vetorial unitário tangente ao segundo fator $\mathbb{R}$. Definimos o campo vetorial diferenciável $T \in T M^{n}$ e a função diferenciável $\nu$ por

$$
\partial / \partial t=d f(T)+\nu \eta
$$


Uma vez que $\partial / \partial t$ é um campo unitário, temos

$$
\nu^{2}+\|T\|^{2}=1
$$

Duas classes triviais de hipersuperfícies em $\mathbb{Q}_{c}^{n} \times \mathbb{R}$ são obtidas se $T \equiv 0$ ou $\nu \equiv 0$, como mostra o seguinte resultado apresentado em [19].

Proposição 1.1.1. Seja $f: M^{n} \rightarrow \mathbb{Q}_{c}^{n} \times \mathbb{R}$ uma hipersuperfície.

(i) Se T é identicamente nulo, então $f\left(M^{n}\right)$ é um subconjunto aberto de $\mathbb{Q}_{c}^{n} \times\{t\}$, para algum $t \in \mathbb{R}$.

(ii) Se $\nu$ é identicamente nulo, então $f\left(M^{n}\right)$ é um subconjunto aberto de um produto Riemanniano $M^{n-1} \times \mathbb{R}$, onde $M^{n-1}$ é uma hipersuperfície de $\mathbb{Q}_{c}^{n}$.

Sejam $\nabla$ a conexão Riemanniana e $R$ o tensor curvatura de $M^{n}$, seja $A$ o operador de Weingarten de $f$ com respeito a $\eta$.

Lembrando que $(X \wedge Y) Z=\langle Y, Z\rangle X-\langle X, Z\rangle Y$, temos as equações de estrutura a seguir.

Proposição 1.1.2. Seja $f: M^{n} \rightarrow \mathbb{Q}_{c}^{n} \times \mathbb{R}$ uma hipersuperfície. As equações de Gauss e Codazzi de $f$ são, para $X, Y, Z \in T M^{n}$, respectivamente,

$$
\begin{gathered}
R(X, Y) Z=(A X \wedge A Y) Z+c((X \wedge Y) Z-\langle Y, T\rangle(X \wedge T) Z+\langle X, T\rangle(Y \wedge T) Z), \\
\left(\nabla_{X} A\right) Y-\left(\nabla_{Y} A\right) X=c \nu(X \wedge Y) T .
\end{gathered}
$$

Além disso, temos, para todo $X \in T M^{n}$, que

$$
\begin{aligned}
& \nabla_{X} T=\nu A X \quad e \\
& X(\nu)=-\langle A X, T\rangle .
\end{aligned}
$$

Demonstração. Observe que para todo $X \in T M^{n}$ temos $d f(X) \in T\left(\mathbb{Q}_{c}^{n} \times \mathbb{R}\right)$ e portanto podemos escrever

$$
\begin{aligned}
d f(X) & =X_{\mathbb{Q}}+\langle d f(X), \partial / \partial t\rangle \partial / \partial t \\
& =X_{\mathbb{Q}}+\langle d f(X), d f(T)+\nu \eta\rangle \partial / \partial t \\
& =X_{\mathbb{Q}}+\langle X, T\rangle \partial / \partial t,
\end{aligned}
$$

onde $X_{\mathbb{Q}}$ é a componente de $d f(X)$ em $T \mathbb{Q}_{c}^{n}$. Logo,

$$
\begin{aligned}
\langle X, Y\rangle & =\langle d f(X), d f(Y)\rangle=\left\langle X_{\mathbb{Q}}+\langle X, T\rangle \partial / \partial t, Y_{\mathbb{Q}}+\langle Y, T\rangle \partial / \partial t\right\rangle \\
& =\left\langle X_{\mathbb{Q}}, Y_{\mathbb{Q}}\right\rangle+\langle X, T\rangle\langle Y, T\rangle
\end{aligned}
$$


o que implica em

$$
\left\langle X_{\mathbb{Q}}, Y_{\mathbb{Q}}\right\rangle=\langle X, Y\rangle-\langle X, T\rangle\langle Y, T\rangle
$$

Sejam $\bar{\nabla}, \nabla^{\mathbb{Q}}$ e $\nabla^{\mathbb{R}}$ as conexões Riemannianas e $\bar{R}, R_{\mathbb{Q}}, R_{\mathbb{R}}$ os tensores curvatura em $\mathbb{Q}_{c}^{n} \times \mathbb{R}, \mathbb{Q}_{c}^{n}$ e $\mathbb{R}$, respectivamente. Então $\bar{\nabla}_{X} Y=\nabla_{X_{\mathbb{Q}}}^{\mathbb{Q}} Y_{\mathbb{Q}}+\nabla_{X_{\mathbb{R}}}^{\mathbb{R}} Y_{\mathbb{R}}$, onde $X_{\mathbb{R}}$ é a componente do campo $d f(X)$ em $\mathbb{R}$. Assim, sendo $\bar{X}=d f(X)$ para todo $X \in T M^{n}$ temos que $\bar{R}(\bar{X}, \bar{Y}) \bar{Z}=R_{\mathbb{Q}}\left(X_{\mathbb{Q}}, Y_{\mathbb{Q}}\right) Z_{\mathbb{Q}}+R_{\mathbb{R}}\left(X_{\mathbb{R}}, Y_{\mathbb{R}}\right) Z_{\mathbb{R}}$. Como $R_{\mathbb{R}}=0$ e $\mathbb{Q}_{c}^{n}$ possui curvatura seccional constante igual a $c$, segue que $\bar{R}(\bar{X}, \bar{Y}) \bar{Z}=c\left(X_{\mathbb{Q}} \wedge Y_{\mathbb{Q}}\right) Z_{\mathbb{Q}}$. Então, utilizando (1.7), obtemos

$$
\begin{aligned}
\langle\bar{R}(\bar{X}, \bar{Y}) \bar{Z}, \bar{W}\rangle= & c\left(\left(X_{\mathbb{Q}} \wedge Y_{\mathbb{Q}}\right) Z_{\mathbb{Q}}, W_{\mathbb{Q}}\right\rangle \\
= & c\left(\left\langle Y_{\mathbb{Q}}, Z_{\mathbb{Q}}\right\rangle\left\langle X_{\mathbb{Q}}, W_{\mathbb{Q}}\right\rangle-\left\langle X_{\mathbb{Q}}, Z_{\mathbb{Q}}\right\rangle\left\langle Y_{\mathbb{Q}}, W_{\mathbb{Q}}\right\rangle\right) \\
= & c(\langle Y, Z\rangle-\langle Y, T\rangle\langle Z, T\rangle)(\langle X, W\rangle-\langle X, T\rangle\langle W, T\rangle)+ \\
& -c(\langle X, Z\rangle-\langle X, T\rangle\langle Z, T\rangle)(\langle Y, W\rangle-\langle Y, T\rangle\langle W, T\rangle) \\
= & c(\langle(X \wedge Y) Z, W\rangle+\langle X, T\rangle\langle(Y \wedge T) Z, W\rangle-\langle Y, T\rangle\langle(X \wedge T) Z, W\rangle) .
\end{aligned}
$$

Sabemos que a equação de Gauss para uma hipersuperfície $f: M^{n} \rightarrow \bar{M}^{n+1}$ é

$$
R(X, Y) Z=\bar{R}(\bar{X}, \bar{Y}) \bar{Z}+(A X \wedge A Y) Z
$$

Portanto a equação de Gauss quando $f: M^{n} \rightarrow \mathbb{Q}_{c}^{n} \times \mathbb{R}$ é dada por (1.3).

A equação de Codazzi para uma hipersuperfície $f: M^{n} \rightarrow \bar{M}^{n+1}$ é

$$
\langle\bar{R}(\bar{X}, \bar{Y}) \bar{Z}, \eta\rangle=\left\langle\left(\nabla_{X} A\right) Y-\left(\nabla_{Y} A\right) X, Z\right\rangle
$$

Observe que

$$
\begin{aligned}
\langle\bar{R}(\bar{X}, \bar{Y}) \bar{Z}, \eta\rangle & =c\left\langle\left(X_{\mathbb{Q}} \wedge Y_{\mathbb{Q}}\right) Z_{\mathbb{Q}}, \eta\right\rangle \\
& =c\left(\left\langle Y_{\mathbb{Q}}, Z_{\mathbb{Q}}\right\rangle\langle d f(X)-\langle X, T\rangle \partial / \partial t, \eta\rangle-\left\langle X_{\mathbb{Q}}, Z_{\mathbb{Q}}\right\rangle\langle d f(Y)-\langle Y, T\rangle \partial / \partial t, \eta\rangle\right) \\
& =c\left(-\nu\left\langle Y_{\mathbb{Q}}, Z_{\mathbb{Q}}\right\rangle\langle X, T\rangle+\nu\left\langle X_{\mathbb{Q}}, Z_{\mathbb{Q}}\right\rangle\langle Y, T\rangle\right) \\
& =c \nu\langle(X \wedge Y) T, Z\rangle .
\end{aligned}
$$

Logo, a equação de Codazzi para $f: M^{n} \rightarrow \mathbb{Q}_{c}^{n} \times \mathbb{R}$ é (1.4). O campo $\partial / \partial t$ é paralelo e portanto

$$
0=\bar{\nabla}_{X} \partial / \partial t=\bar{\nabla}_{X}(d f(T)+\nu \eta)=\bar{\nabla}_{X} d f(T)+\alpha(X, T)+X(\nu) \eta-\nu d f\left(A_{\eta} X\right)
$$

Daí seguem (1.5) e (1.6).

Observação 1.1.3. Pela igualdade (1.6), se a função $\nu$ é constante e $T \neq 0$, então o campo 
$T$ é uma direção principal associada ao autovalor 0, ou seja, $A T=0$.

Considerando $F: M^{n} \rightarrow \mathbb{E}^{n+2}$ como $F:=i \circ f$ onde $i: \mathbb{Q}_{c}^{n} \times \mathbb{R} \rightarrow \mathbb{E}^{n+2}$ é a aplicação inclusão com normal $\xi$ tal que $\langle\xi, \xi\rangle=c$, obtemos $A_{\xi}(T)=-\nu^{2} T$ e $A_{\xi}(X)=-X$, para todo $X \in[T]^{\perp}$ onde $[T]^{\perp}=\left\{X \in T M^{n} /\langle X, T\rangle=0\right\}$.

O seguinte resultado fornece uma condição sobre o campo $T$ para que $F: M^{n} \rightarrow \mathbb{E}^{n+2}$, onde $F:=i \circ f$, tenha o fibrado normal plano.

Proposição 1.1.4. Seja $f: M^{n} \rightarrow \mathbb{Q}_{c}^{n} \times \mathbb{R}$ uma hipersuperfície. Suponha que $T(p) \neq 0$, para algum $p \in M^{n}$. Então $f$ tem fibrado normal plano em $p$ como uma imersão isométrica em $\mathbb{E}^{n+2}$ se e somente se $T$ é uma direção principal em $p$.

A seguir vamos apresentar algumas famílias especiais de hipersuperfícies de $\mathbb{Q}_{c}^{n} \times \mathbb{R}$.

\subsubsection{Hipersuperfícies totalmente geodésicas}

Nesta subseção abordamos o estudo das hipersuperfícies de $\mathbb{Q}_{c}^{n} \times \mathbb{R}$ totalmente geodésicas. Em [33] foi dado um teorema que classifica tais hipersuperfícies em $\mathbb{S}^{n} \times \mathbb{R}$. O mesmo resultado vale também para $\mathbb{H}^{n} \times \mathbb{R}$, como é mostrado a seguir.

Teorema 1.1.5. Seja $f: M^{n} \rightarrow \mathbb{Q}_{c}^{n} \times \mathbb{R}$ uma hipersuperfície totalmente geodésica. Então $f\left(M^{n}\right)$ é um aberto de $\mathbb{Q}_{c}^{n} \times\left\{t_{0}\right\}$, para algum $t_{0} \in \mathbb{R}$ ou de $M^{n-1} \times \mathbb{R}$, onde $M^{n-1}$ é uma hipersuperficie totalmente geodésica de $\mathbb{Q}_{c}^{n}$.

Demonstração. Seja $f$ uma hipersuperfície totalmente geodésica, então, pela equação de Codazzi (1.4), temos

$$
0=\left(\nabla_{X} A\right) Y-\left(\nabla_{Y} A\right) X=c \nu(\langle Y, T\rangle X-\langle X, T\rangle Y)
$$

para todo $X, Y \in T M^{n}$. Dessa forma, temos dois casos a considerar: $\nu=0$ e $T=0$.

Se $T=0$, então, pela Proposição 1.1.1, a hipersuperfície é uma fatia $\mathbb{Q}_{c}^{n} \times\left\{t_{0}\right\}$ de $\mathbb{Q}_{c}^{n} \times \mathbb{R}$, para algum $t_{0} \in \mathbb{R}$.

Se $\nu$ é identicamente nulo, então $f\left(M^{n}\right)$ é um subconjunto aberto de um produto Riemanniano $M^{n-1} \times \mathbb{R}$, onde $M^{n-1}$ é uma hipersuperfície de $\mathbb{Q}_{c}^{n}$. Observe que $M^{n-1} \times \mathbb{R}$ é totalmente geodésica em $\mathbb{Q}_{c}^{n} \times \mathbb{R}$ se, e somente se, $M^{n-1}$ é totalmente geodésica em $\mathbb{Q}_{c}^{n}$.

\subsubsection{Hipersuperfícies rotacionais}

Nesta subseção introduzimos a noção de hipersuperfícies de rotação em $\mathbb{Q}_{c}^{n} \times \mathbb{R}$, cuja definição foi dada em [15] como uma extensão do trabalho das hipersuperfícies de rotação em 
espaços forma, estudados em [16]. Os resultados aqui apresentados podem ser encontrados com maiores detalhes em [15].

Considere um subespaço tridimensional $P^{3}$ de $\mathbb{E}^{n+2}$ contendo o $x_{n+2}$-eixo. Dessa forma, $\left(\mathbb{Q}_{c}^{n} \times \mathbb{R}\right) \cap P^{3}=\mathbb{Q}_{c}^{1} \times \mathbb{R}$. Seja $P^{2}$ um subespaço de $P^{3}$ que também contém o $x_{n+2}$-eixo. Denotaremos por $\mathcal{I}$ o grupo de isometrias de $\mathbb{E}^{n+2}$ que deixa $\mathbb{Q}_{c}^{n} \times \mathbb{R}$ globalmente invariante e deixa $P^{2}$ fixado ponto a ponto. Seja $\alpha$ uma curva em $\mathbb{Q}_{c}^{1} \times \mathbb{R}$ que não intersecta $P^{2}$.

Definição 1.1.6. Uma hipersuperfície de rotação $f: M^{n} \rightarrow \mathbb{Q}_{c}^{n} \times \mathbb{R}$ gerada pela curva $\alpha e$ com eixo $P^{2}$ é definida como a $\mathcal{I}$-órbita de $\alpha$.

As hipersuperfícies rotacionais de $\mathbb{S}^{n} \times \mathbb{R}$ são folheadas por esferas. As rotacionais de $\mathbb{H}^{n} \times \mathbb{R}$ são folheadas por esferas se $P^{2}$ é Lorentziano, por espaços hiperbólicos se $P^{2}$ é Riemanniano e por horosferas se $P^{2}$ é degenerado. No caso em que a curva $\alpha$ está em um plano ortogonal a $\frac{\partial}{\partial x_{n+2}}$ temos que $T=0$ e caso contrário, o vetor velocidade de $\alpha$ tem componentes na direção de $T$. A fim de apresentar uma parametrização explícita de uma hipersuperfície rotacional vamos considerar quatro casos e em todos eles assumiremos que $P^{3}$ é gerado por $\frac{\partial}{\partial x_{1}}, \frac{\partial}{\partial x_{n+1}}$ e $\frac{\partial}{\partial x_{n+2}}$.

Caso 1: $c=1$

Suponha que $P^{2}$ é gerado por $\frac{\partial}{\partial x_{1}}$ e $\frac{\partial}{\partial x_{n+2}}$. Primeiramente consideramos o caso em que a curva geratriz não é uma reta vertical em $\mathbb{S}^{1} \times \mathbb{R}$. Então $\alpha$ pode ser parametrizada como

$$
\alpha(s)=(\cos (s), 0, \ldots, 0, \operatorname{sen}(s), a(s))
$$

para uma certa função $a$. Uma vez que $\alpha$ não intersecta $P^{2}$ podemos escolher o intervalo de parametrização $I$, tal que a função $\operatorname{sen}(s)$ nunca se anule. Observe que se $a(s)$ é uma função constante temos que $a^{\prime}(s) \equiv 0$ e, nesse caso, a curva $\alpha$ está em um plano ortogonal a $\frac{\partial}{\partial x_{n+2}}$ e $T=0$. Caso contrário, podemos supor $a^{\prime}(s)>0$, para todo $s \in I$.

Uma parametrização explícita da hipersuperfície de rotação é dada por

$$
f\left(s, t_{1}, \ldots, t_{n-1}\right)=\left(\cos (s), \operatorname{sen}(s) \varphi_{1}\left(t_{1}, \ldots, t_{n-1}\right), \ldots, \operatorname{sen}(s) \varphi_{n}\left(t_{1}, \ldots, t_{n-1}\right), a(s)\right)
$$

onde $\varphi=\left(\varphi_{1}, \ldots, \varphi_{n}\right)$ é uma parametrização ortogonal da esfera unitária $\mathbb{S}^{n-1}$ em $\mathbb{R}^{n}$, ou seja, $\varphi_{1}^{2}+\ldots+\varphi_{n}^{2}=1$ e $\frac{\partial \varphi_{1}}{\partial t_{i}} \frac{\partial \varphi_{1}}{\partial t_{j}}+\ldots+\frac{\partial \varphi_{n}}{\partial t_{i}} \frac{\partial \varphi_{n}}{\partial t_{j}}=\delta_{i j}\left\|\frac{\partial \varphi}{\partial t_{i}}\right\|^{2}$. Denotamos $\left(s, t_{1}, \ldots, t_{n}\right)$ por 
$(s, t)$, onde $t=\left(t_{1}, \ldots, t_{n}\right)$. Observe que

$$
\begin{aligned}
\frac{\partial f}{\partial s}(s, t) & =\left(-\operatorname{sen}(s), \cos (s) \varphi_{1}(t), \ldots, \cos (s) \varphi_{n}(t), a^{\prime}(s)\right) \\
\frac{\partial f}{\partial t_{i}}(s, t) & =\left(0, \operatorname{sen}(s) \frac{\partial \varphi_{1}}{\partial t_{i}}(t), \ldots, \operatorname{sen}(s) \frac{\partial \varphi_{n}}{\partial t_{i}}(t), 0\right) \\
\xi(s, t) & \left.=\left(\cos (s), \operatorname{sen}(s) \varphi_{1}(t), \ldots, \operatorname{sen}(s) \varphi_{n}(t), 0\right)\right)
\end{aligned}
$$

Portanto o campo unitário $\eta$ normal à $f$ é dado por

$$
\eta(s, t)=\frac{1}{\sqrt{1+a^{\prime}(s)^{2}}}\left(-\operatorname{sen}(s) a^{\prime}(s), \cos (s) a^{\prime}(s) \varphi_{1}(t), \ldots, \cos (s) a^{\prime}(s) \varphi_{n}(t),-1\right) .
$$

Para calcular o operador de Weingarten $A$ de $f$, observe que, para todo $X, Y \in T M^{n}$, temos

$$
\langle A X, Y\rangle=\left\langle-\bar{\nabla}_{X} \eta, Y\right\rangle=\left\langle\bar{\nabla}_{X} Y, \eta\right\rangle=\left\langle\widehat{\nabla}_{X} Y, \eta\right\rangle
$$

onde $\widehat{\nabla}$ é a conexão Riemanniana de $\mathbb{E}^{n+2}$. Uma vez que $\varphi$ é uma parametrização ortogonal da esfera unitária, para $i \neq j$, obtemos

$$
\begin{aligned}
\left\langle A \frac{\partial f}{\partial t_{i}}, \frac{\partial f}{\partial t_{j}}\right\rangle & =\left\langle\frac{\partial^{2} f}{\partial t_{i} \partial t_{j}}, \eta\right\rangle=0, \\
\left\langle A \frac{\partial f}{\partial t_{i}}, \frac{\partial f}{\partial s}\right\rangle & =\left\langle\frac{\partial^{2} f}{\partial t_{i} \partial s}, \eta\right\rangle=0 .
\end{aligned}
$$

Dessa forma, em cada ponto $(s, t)$, a base $\left\{\frac{\partial f}{\partial s}, \frac{\partial f}{\partial t_{1}}, \ldots, \frac{\partial f}{\partial t_{n-1}}\right\}$ diagonaliza o operador A. Então as curvaturas principais são dadas por

$$
\begin{gathered}
\lambda=\left\langle A \frac{\partial f}{\partial s}, \frac{\partial f}{\partial s}\right\rangle \frac{1}{\left\langle\frac{\partial f}{\partial s}, \frac{\partial f}{\partial s}\right\rangle}=\left\langle\frac{\partial^{2} f}{\partial s^{2}}, \eta\right\rangle \frac{1}{1+a^{\prime}(s)^{2}}=-\frac{a^{\prime \prime}(s)}{\left(1+a^{\prime}(s)^{2}\right)^{\frac{3}{2}}} \\
\mu_{i}=\left\langle A \frac{\partial f}{\partial t_{i}}, \frac{\partial f}{\partial t_{i}}\right\rangle \frac{1}{\left\langle\frac{\partial f}{\partial t_{i}}, \frac{\partial f}{\partial t_{i}}\right\rangle}=\left\langle\frac{\partial^{2} f}{\partial t_{i}^{2}}, \eta\right\rangle \frac{1}{\operatorname{sen}^{2}(s)\left\|\frac{\partial \varphi}{\partial t_{i}}\right\|^{2}}=-\frac{a^{\prime}(s) \cot (s)}{\left(1+a^{\prime}(s)^{2}\right)^{\frac{1}{2}}}
\end{gathered}
$$

Como $\mu_{i}$ não depende de $i$, o denotaremos por $\mu$.

Se $\alpha$ é uma reta vertical $\alpha(s)=(\cos (k), 0, \ldots, 0, \operatorname{sen}(k), s)$, onde $k$ é uma constante real tal que $\operatorname{sen}(k) \neq 0$. Obtemos, de maneira análoga à anterior, as curvaturas principais $\lambda=0$ e $\mu=-\cot (k)$.

Concluimos assim que o operador de Weingarten $A$ possui, no máximo, dois autovalores distintos e se existirem exatamente dois, um deles tem multiplicidade 1 e o correspondente 
autoespaço é gerado por $T$.

Caso 2: $c=-1$ e $P^{2}$ é Lorentziano (as órbitas são esferas)

Suponha que $P^{2}$ é gerado por $\frac{\partial}{\partial x_{1}}$ e $\frac{\partial}{\partial x_{n+2}}$. Considere a curva

$$
\alpha(s)=(\cosh (s), 0, \ldots, 0, \operatorname{senh}(s), a(s)), \quad \operatorname{com} s \neq 0 .
$$

Fazendo um cálculo análogo ao do Caso 1, obtemos

$$
\lambda=-\frac{a^{\prime \prime}(s)}{\left(1+a^{\prime}(s)^{2}\right)^{\frac{3}{2}}} \quad \text { e } \quad \mu=-\frac{a^{\prime}(s) \operatorname{coth}(s)}{\left(1+a^{\prime}(s)^{2}\right)^{\frac{1}{2}}} .
$$

Se $\alpha$ é uma reta vertical $\alpha(s)=(\cosh (k), 0, \ldots, 0, \operatorname{senh}(k), s)$, onde $k \neq 0$ é uma constante real, temos que $\lambda=0$ e $\mu=-\operatorname{coth}(k)$.

Caso 3: $c=-1$ e $P^{2}$ é Riemanniano (as órbitas são espaços hiperbólicos)

Suponha que $P^{2}$ é gerado por $\frac{\partial}{\partial x_{n+1}}$ e $\frac{\partial}{\partial x_{n+2}}$ e que a curva geratriz é dada por

$$
\alpha(s)=(\cosh (s), 0, \ldots, 0, \operatorname{senh}(s), a(s)) .
$$

Observe que $\alpha$ não intersecta $P^{2}$, já que $\cosh (s) \neq 0$. Uma parametrização explícita para a hipersuperfície de rotação é dada por

$$
f\left(s, t_{1}, \ldots, t_{n-1}\right)=\left(\cosh (s) \varphi_{1}\left(t_{1}, \ldots, t_{n-1}\right), \ldots, \cosh (s) \varphi_{n}\left(t_{1}, \ldots, t_{n-1}\right), \operatorname{senh}(s), a(s)\right),
$$

onde $\varphi=\left(\varphi_{1}, \ldots, \varphi_{n}\right)$ é uma parametrização ortogonal do espaço hiperbólico $\mathbb{H}^{n-1}$ em $\mathbb{L}^{n}$, ou seja,

$$
-\varphi_{1}^{2}+\varphi_{2}^{2}+\ldots+\varphi_{n}^{2}=-1, \quad \varphi_{1}>0 \quad \text { e } \quad-\frac{\partial \varphi_{1}}{\partial t_{i}} \frac{\partial \varphi_{1}}{\partial t_{j}}+\ldots+\frac{\partial \varphi_{n}}{\partial t_{i}} \frac{\partial \varphi_{n}}{\partial t_{j}}=\delta_{i j}\left\|\frac{\partial \varphi}{\partial t_{i}}\right\|^{2}
$$

Assim,

$$
\begin{aligned}
\frac{\partial f}{\partial s}(s, t) & =\left(\operatorname{senh}(s) \varphi_{1}(t), \ldots, \operatorname{senh}(s) \varphi_{n}(t), \cosh (s), a^{\prime}(s)\right) \\
\frac{\partial f}{\partial t_{i}}(s, t) & =\left(\cosh (s) \frac{\partial \varphi_{1}}{\partial t_{i}}(t), \ldots, \cosh (s) \frac{\partial \varphi_{n}}{\partial t_{i}}(t), 0,0\right) \\
\xi(s, t) & \left.=\left(\cosh (s) \varphi_{1}(t), \ldots, \cosh (s) \varphi_{n}(t), \operatorname{senh}(s), 0\right)\right) .
\end{aligned}
$$

Portanto,

$$
\eta(s, t)=\frac{1}{\sqrt{1+a^{\prime}(s)^{2}}}\left(\operatorname{senh}(s) a^{\prime}(s) \varphi_{1}(t), \ldots, \operatorname{senh}(s) a^{\prime}(s) \varphi_{n}(t), \cosh (s) a^{\prime}(s),-1\right) .
$$


Temos que, para cada ponto $(s, t)$, a base $\left\{\frac{\partial f}{\partial s}, \frac{\partial f}{\partial t_{1}}, \ldots, \frac{\partial f}{\partial t_{n-1}}\right\}$ diagonaliza o operador $A$. As curvaturas principais são dadas por

$$
\lambda=-\frac{a^{\prime \prime}(s)}{\left(1+a^{\prime}(s)^{2}\right)^{\frac{3}{2}}}
$$

com $A T=\lambda T \mathrm{e}$

$$
\mu=-\frac{a^{\prime}(s) \tanh (s)}{\left(1+a^{\prime}(s)^{2}\right)^{\frac{1}{2}}} .
$$

No caso em que $\alpha$ é uma reta vertical $\alpha(s)=(\cosh (k), 0, \ldots, 0, \operatorname{senh}(k), s) \operatorname{com} k \in \mathbb{R}$, temos $\lambda=0$ e $\mu=-\tanh (k)$.

Caso 4: $c=-1$ e $P^{2}$ é degenerado (as órbitas são horosferas)

Nesse caso, consideramos a seguinte base pseudo-ortonormal de $\mathbb{L}^{n+2}$

$$
e_{1}=\frac{1}{\sqrt{2}}\left(-\frac{\partial}{\partial x_{1}}+\frac{\partial}{\partial x_{n+1}}\right), \quad e_{n+1}=\frac{1}{\sqrt{2}}\left(\frac{\partial}{\partial x_{1}}+\frac{\partial}{\partial x_{n+1}}\right), \quad e_{i}=\frac{\partial}{\partial x_{i}}
$$

para $i \in\{2, \ldots, n, n+2\}$ e supomos que que $P^{2}$ é gerado por $e_{n+1}$ e $e_{n+2}$. Observe que $\left\langle e_{1}, e_{1}\right\rangle=\left\langle e_{n+1}, e_{n+1}\right\rangle=0$ e $\left\langle e_{1}, e_{n+1}\right\rangle=1$. Se $\alpha$ não é uma reta vertical, consideramos

$$
\alpha(s)=\left(s, 0, \ldots, 0,-\frac{1}{2 s}, a(s)\right)
$$

com respeito a base $\left\{e_{1}, \ldots, e_{n+2}\right\}$. Em [16], foi mostrado que, neste caso, o grupo $\mathcal{I}$ consiste de transformações da forma $A_{(t, i)}$, com $t \in \mathbb{R}, i \in\{2, \ldots, n\}$, cuja ação em $\alpha$ é dada por

$$
A_{(t, i)} \alpha(s)=(s, 0, \ldots, 0, \underbrace{t s}_{i}, 0, \ldots, 0,-\frac{1}{2 s}-s \frac{t^{2}}{2}, a(s)) .
$$

A parametrização da hipersuperfície de rotação é dada por

$$
f\left(s, t_{2}, \ldots, t_{n}\right)=\left(s, s t_{2}, \ldots, s t_{n},-\frac{1}{2 s}-\frac{s}{2} \sum_{i=2}^{n} t_{i}^{2}, a(s)\right)
$$

Denotando $(s, t)$ por $\left(s, t_{2}, \ldots, t_{n}\right)$, obtemos: 


$$
\begin{aligned}
\frac{\partial f}{\partial s}(s, t) & =\left(1, t_{2}, \ldots, t_{n}, \frac{1}{2 s^{2}}-\frac{1}{2} \sum_{i=2}^{n} t_{i}^{2}, a^{\prime}(s)\right), \\
\frac{\partial f}{\partial t_{i}}(s, t) & =(0,0, \ldots, 0, \underbrace{s}_{i}, 0, \ldots,-s t_{i}, 0), \\
\xi(s, t) & =\left(s, s t_{2}, \ldots, s t_{n},-\frac{1}{2 s}-\frac{s}{2} \sum_{i=2}^{n} t_{i}^{2}, 0\right), \\
\eta(s, t)= & \frac{1}{\sqrt{\frac{1}{s^{2}}+a^{\prime}(s)^{2}}}\left(s a^{\prime}(s), s a^{\prime}(s) t_{2}, \ldots, s a^{\prime}(s) t_{n}, \frac{1}{2 s} a^{\prime}(s)-\frac{s}{2} a^{\prime}(s) \sum_{i=2}^{n} t_{i}^{2},-\frac{1}{s}\right) .
\end{aligned}
$$

As curvaturas principais são dadas por

$$
\lambda=-\frac{s a^{\prime}(s)+s^{2} a^{\prime \prime}(s)}{\left(1+s^{2} a^{\prime}(s)^{2}\right)^{\frac{3}{2}}} \quad \text { e } \quad \mu=-\frac{s a^{\prime}(s)}{\left(1+s^{2} a^{\prime}(s)^{2}\right)^{\frac{1}{2}}},
$$

onde $\lambda$ possui, em geral, multiplicidade 1 e a direção principal associada a $\lambda$ é $T$.

Observe que, fazendo a reparametrização $s=e^{r} \operatorname{com} \tilde{a}(r)=a\left(e^{r}\right)=a(s)$, obtemos $\tilde{a}^{\prime}(r)=e^{r} a^{\prime}\left(e^{r}\right)=s a^{\prime}(s)$ e $\quad \tilde{a}^{\prime \prime}(r)=e^{r} a^{\prime}\left(e^{r}\right)+\left(e^{r}\right)^{2} a^{\prime \prime}\left(e^{r}\right)=s a^{\prime}(s)+s^{2} a^{\prime \prime}(s)$. Ou seja, podemos reescrever

$$
\lambda=-\frac{\tilde{a}^{\prime \prime}(r)}{\left(1+\left(\tilde{a}^{\prime}(r)\right)^{2}\right)^{\frac{3}{2}}} \quad \text { e } \quad \mu=-\frac{\tilde{a}^{\prime}(r)}{\left(1+\left(\tilde{a}^{\prime}(r)\right)^{2}\right)^{\frac{1}{2}}}
$$

Se $\alpha$ é uma reta vertical, $\alpha(s)=\left(k, 0, \ldots, 0,-\frac{1}{2 k}, s\right)$ com $k \in \mathbb{R}$, obtemos

$$
\lambda=0 \quad \text { e } \quad \mu=-1 .
$$

Observação 1.1.7. Quando a curva geratriz é uma reta vertical então a função $\nu \equiv 0$. De fato, para $c=1$, se $\alpha(s)=(\cos (k), 0, \ldots, 0, \operatorname{sen}(k), s)$, onde $k$ é uma constante real tal que $\operatorname{sen}(k) \neq 0$, a parametrização é $f(s, t)=\left(\cos (k), \operatorname{sen}(k) \varphi_{1}(t), \ldots, \operatorname{sen}(k) \varphi_{n}(t), s\right)$. Logo, $\frac{\partial f}{\partial s}=(0,0, \ldots, 0,1)=\partial / \partial t$, ou seja, $\partial / \partial t$ é tangente a $f$ e portanto $\nu \equiv 0$. Para $c=-1$, de maneira análoga concluimos que $\nu \equiv 0$, quando a curva geratriz é uma reta vertical.

Se $T=0$, então a hipersuperfície é totalmente geodésica e, portanto, as curvaturas principais são todas nulas.

Quando a curva geratriz não é uma reta vertical temos que

$$
\nu(s)=\langle\eta, \partial / \partial t\rangle=-\frac{1}{\sqrt{1+a^{\prime}(s)^{2}}} \neq 0
$$


para todo s, e para o Caso $4, \nu(s)=-\frac{1}{\sqrt{1+s^{2} a^{\prime}(s)^{2}}} \neq 0$ para todo $s$, ou seja, a função $\nu$ nunca se anula.

Pelo próximo teorema, uma hipersuperfície rotacional $f: M^{n} \rightarrow \mathbb{Q}_{c}^{n} \times \mathbb{R}$ possui o fibrado normal plano quando considerada como subvariedade de codimensão dois em $\mathbb{E}^{n+2}$. Assim, pela Proposição 1.1.4, isso é equivalente a dizer que as hipersuperfícies rotacionais possuem o campo $T$ como uma direção principal.

Teorema 1.1.8 (Teorema 5 de [15]). Seja $f: M^{n} \rightarrow \mathbb{Q}_{c}^{n} \times \mathbb{R}$ uma hipersuperfície de rotação. Então $f$ tem fibrado normal plano em $\mathbb{E}^{n+2}$.

O resultado seguinte estabelece um critério para uma hipersuperfície em $\mathbb{Q}_{c}^{n} \times \mathbb{R}$, com $n \geq 3$, ser de rotação.

Teorema 1.1.9 (Teorema 2 de [15]). Seja $f: M^{n} \rightarrow \mathbb{Q}_{c}^{n} \times \mathbb{R}, n \geq 3$, uma hipersuperfície com operador de Weingaten

$$
A=\left(\begin{array}{cccc}
\lambda & & & \\
& \mu & & \\
& & \ddots & \\
& & & \mu
\end{array}\right)
$$

com $\lambda \neq \mu$. Suponha que $A T=\lambda T$ e que existe uma relação funcional $\lambda(\mu)$. Então $f\left(M^{n}\right) e^{\prime}$ um subconjunto aberto de uma hipersuperfície de rotação.

Em [19], foi dado um outro critério para uma hipersuperfície em $\mathbb{Q}_{c}^{n} \times \mathbb{R}$ ser de rotação sem a hipótese da existência de uma relação funcional entre $\lambda$ e $\mu$.

Proposição 1.1.10. (Proposição 7.3 de [19]) Seja $f: M^{n} \rightarrow \mathbb{Q}_{c}^{n} \times \mathbb{R}, n \geq 3$, uma hipersuperfície com $T \neq 0$. Suponha que $f$ possui exatamente duas curvaturas principais $\lambda$ e $\mu$, com $\lambda$ de multiplicidade 1 e $T$ uma direção principal associada a $\lambda$. Se $\lambda$ é constante ao longo das folhas dos autofibrados $\{T\}^{\perp}$ de $\mu$, então $f\left(M^{n}\right)$ é um subconjunto aberto de uma hipersuperfície de rotação.

\subsubsection{Hipersuperfícies que possuem o campo $T$ como uma direção principal}

Os resultados desta subseção foram apresentados e demonstrados em [32]. Eles descrevem hipersuperfícies $f: M^{n} \rightarrow \mathbb{Q}_{c}^{n} \times \mathbb{R}$ com fibrado normal plano, quando consideradas como subvariedades de codimensão 2 em $\mathbb{E}^{n+2}$, ou seja, hipersuperfícies que possuem o campo $T$ 
como uma direção principal. Em particular, estão incluidas neste estudo as hipersuperfícies de ângulo constante, isto é, quando a função $\nu$ é constante.

Seja $h: M^{n-1} \rightarrow \mathbb{Q}_{c}^{n}$ uma hipersuperfície e seja $h_{s}: M^{n-1} \rightarrow \mathbb{Q}_{c}^{n}$ uma família de hipersuperfícies paralelas dadas por $h_{s}(x)=C_{c}(s) h(x)+S_{c}(x) N(x)$, para cada $x \in M^{n-1}$, onde $N$ é o campo normal unitário a $h$ e

$$
C_{c}(s)=\left\{\begin{array}{cll}
\cos (s), & \text { se } & c=1 \\
1, & \text { se } & c=0 \\
\cosh (s), & \text { se } & c=-1
\end{array} \quad, \quad S_{c}(s)=\left\{\begin{array}{cll}
\operatorname{sen}(s), & \text { se } & c=1 \\
s, & \text { se } & c=0 \\
\operatorname{senh}(s), & \text { se } & c=-1
\end{array}\right.\right.
$$

Defina $f: M^{n}=M^{n-1} \times I \rightarrow \mathbb{Q}_{c}^{n} \times \mathbb{R}$ por

$$
f(x, s)=h_{s}(x)+a(s) \partial / \partial t
$$

onde $I \subset \mathbb{R}$ é um intervalo aberto e $a: I \rightarrow \mathbb{R}$ é uma função diferenciável com $a^{\prime}(s)>0$, para todo $s \in I$.

Teorema 1.1.11. (Teorema 1 de [32]) A aplicação f dada por (1.15) define, nos pontos regulares, uma hipersuperfície que tem $T$ como uma direção principal. Reciprocamente, qualquer hipersuperfície $f: M^{n} \rightarrow \mathbb{Q}_{c}^{n} \times \mathbb{R}$ com $n \geq 2$ e $\nu(p) \neq 0, \forall p \in M^{n}$ que tem $T$ como direção principal é localmente dada por (1.15).

Como uma consequência do Teorema 1.1.11, segue abaixo uma descrição completa de todas hipersuperfícies $f: M^{n} \rightarrow \mathbb{Q}_{c}^{n} \times \mathbb{R}$ com função ângulo constante.

Corolário 1.1.12. (Corolário 2 de [32]) Seja $f$ dada por (1.15) com a $(s)=$ Bs para algum $B>0$. Então $f$ é uma hipersuperfície de ângulo constante. Reciprocamente, qualquer hipersuperfície $f: M^{n} \rightarrow \mathbb{Q}_{c}^{n} \times \mathbb{R}$ com $n \geq 2$ de ângulo constante ou é um subconjunto aberto de $\mathbb{Q}_{c}^{n} \times\left\{t_{0}\right\}$ para algum $t_{0} \in \mathbb{R}$, ou um subconjunto aberto de um produto $M^{n-1} \times \mathbb{R}$, onde $M^{n-1}$ é uma hipersuperfície de $\mathbb{Q}_{c}^{n}$, ou é localmente dada por (1.15).

O próximo resultado caracteriza as hipersuperfícies de curvatura média constante em $\mathbb{Q}_{c}^{n} \times \mathbb{R}$ que possuem o campo $T$ como uma direção principal.

Teorema 1.1.13 (Teorema 3 de [32]). Sejam $h: M^{n-1} \rightarrow \mathbb{Q}_{c}^{n}$ uma hipersuperfície isoparamétrica, $I \subset \mathbb{R}$ um intervalo aberto tal que as hipersuperfícies paralelas $h_{s}$ são imersões para todo $s \in I$ e $H(s)$ a curvatura média constante de $h_{s}$. Dados $H, \phi_{0} \in \mathbb{R}$ e $s_{0} \in I$ com $0<\phi_{0}<1$, seja $I$, se necessário ainda mais restrito, tal que

$$
\phi(s):=(\psi(s))^{-1}\left(\phi_{0}+H \int_{s_{0}}^{s} \psi(\tau) d \tau\right)
$$


$\operatorname{com} \psi(s)=\exp \left(-\int_{s_{0}}^{s} H(\tau) d \tau\right)$, satisfaz $0<\phi(s)<1$ para todo $s \in I$. Defina $a: I \rightarrow \mathbb{R}$ por $a(s)=a_{0}+\int_{s_{0}}^{s} \phi(\tau)\left(1-\phi^{2}(\tau)\right)^{-\frac{1}{2}} d \tau, a_{0} \in \mathbb{R}$. Então $f: M^{n-1} \times I \rightarrow \mathbb{Q}_{c}^{n} \times \mathbb{R} d a d a$ por (1.15) é uma hipersuperfície com curvatura média constante $H$ que possui $T$ como uma direção principal. Reciprocamente, qualquer hipersuperfície $f: M^{n} \rightarrow \mathbb{Q}_{c}^{n} \times \mathbb{R}, n \geq 2$, com $\nu(p) \neq 0$, para todo $p \in M^{n}$ e curvatura média constante $H$ que possui $T$ como uma direção principal é localmente dada desta maneira.

A proposição abaixo descreve as curvaturas principais de uma hipersuperfície dada por (1.15). Denotamos o operador de Weingarten de $h_{s}$ por $A^{s}$.

Proposição 1.1.14. Seja $f: M^{n} \rightarrow \mathbb{Q}_{c}^{n} \times \mathbb{R}$ dada por (1.15). Então, em cada ponto regular $(x, s) \in M^{n-1} \times I$, temos que $A_{\eta} X=-\frac{a^{\prime}(s)}{\sqrt{a^{\prime}(s)^{2}+1}} A^{s} X$, para todo $X \in T M^{n-1} e$ $A_{\eta} T=\frac{a^{\prime \prime}(s)}{\left(a^{\prime}(s)^{2}+1\right)^{\frac{3}{2}}} T$.

Demonstração. Seja $f: M^{n}=M^{n-1} \times I \rightarrow \mathbb{Q}_{c}^{n} \times \mathbb{R}$ dada por (1.15), isto é,

$$
f(x, s)=h_{s}(x)+a(s) \partial / \partial t=C_{c}(s) h(x)+S_{c}(x) N(x)+a(s) \partial / \partial t .
$$

Então, $d f(X)=d h_{s}(X)$, para todo $X \in T M^{n-1}$ e $d f\left(\frac{\partial}{\partial s}\right)=N_{s}+a^{\prime}(s) \frac{\partial}{\partial t}$, onde $N_{s}(x)=-c S_{c}(s) h(x)+C_{c}(s) N(x)$.

Uma vez que $a^{\prime}(s)>0$, temos $d f\left(\frac{\partial}{\partial s}\right) \neq 0$, para todo $s \in I$. Assim, um ponto $(x, s) \in M^{n-1} \times I$ é regular para $f$ se, e somente se, $h_{s}$ é regular em $x$ e nesse caso, $N_{s}(x)$ é um vetor unitário normal a $h_{s}$ em $x$. Além disso,

$$
\eta(x, s)=-\frac{a^{\prime}(s)}{b(s)} N_{s}(x)+\frac{1}{b(s)} \frac{\partial}{\partial t}, \quad \operatorname{com} b(s)=\sqrt{1+a^{\prime}(s)^{2}}
$$

é um vetor unitário normal a $f$ em $(x, s)$. Note que $\left\langle d f(X), d f\left(\frac{\partial}{\partial s}\right)\right\rangle=0$, para todo $X \in T M^{n-1}$. Denotamos por $\widehat{\nabla}$ a conexão Riemanniana de $\mathbb{E}^{n+2}$. Temos que

$$
\widehat{\nabla}_{\frac{\partial}{\partial s}} \eta=-\left(\frac{a^{\prime}(s)}{b(s)}\right)^{\prime} N_{s}+c \frac{a^{\prime}(s)}{b(s)} h_{s}+\left(\frac{1}{b(s)}\right)^{\prime} \frac{\partial}{\partial t}
$$

Então,

$$
\left\langle\widehat{\nabla}_{\frac{\partial}{\partial s}} \eta, d f(X)\right\rangle=\left\langle\widehat{\nabla}_{\frac{\partial}{\partial s}} \eta, d h_{s}(X)\right\rangle=0
$$


para todo $X \in T M^{n-1}$, o que mostra que $\frac{\partial}{\partial s}$ é uma direção principal de $f$. Utilizando que

$$
\nu=\left\langle\eta, \frac{\partial}{\partial t}\right\rangle=\frac{1}{b(s)}
$$

obtemos

$$
d f(T)=\frac{\partial}{\partial t}-\nu \eta=\frac{a^{\prime}(s)}{b^{2}(s)} d f\left(\frac{\partial}{\partial s}\right)
$$

Logo, $T=\frac{a^{\prime}(s)}{b^{2}(s)} \frac{\partial}{\partial s}$. Além disso,

$$
\left\langle\widehat{\nabla}_{\frac{\partial}{\partial s}} \eta, d f\left(\frac{\partial}{\partial s}\right)\right\rangle=-\left(\frac{a^{\prime}(s)}{b(s)}\right)^{\prime}+a^{\prime}(s)\left(\frac{1}{b(s)}\right)^{\prime}=-\frac{a^{\prime \prime}(s)}{b(s)} .
$$

Dessa forma, a curvatura principal associada ao campo $T$ é

$$
\lambda=-\frac{\left\langle\widehat{\nabla}_{\frac{\partial}{\partial s}} \eta, d f\left(\frac{\partial}{\partial s}\right)\right\rangle}{\left\langle d f\left(\frac{\partial}{\partial s}\right), d f\left(\frac{\partial}{\partial s}\right)\right\rangle}=\frac{a^{\prime \prime}(s)}{b(s)^{3}} .
$$

Observe que, para $X \in T M^{n-1}$,

$$
\widehat{\nabla}_{X} \eta=\frac{a^{\prime}(s)}{b(s)} d h_{s}\left(A^{s} X\right)
$$

Logo, o operador $A_{\eta}$ satisfaz em $(x, s)$,

$$
A_{\eta} X=-\frac{a^{\prime}(s)}{b(s)} A^{s} X, \quad \text { para todo } X \in T M^{n-1} .
$$

O próximo resultado relaciona as curvaturas principais de uma hipersuperfície em $\mathbb{Q}_{c}^{n}$ e de suas hipersuperfícies paralelas. Uma prova para o caso $c=1$ pode ser encontrada em [9]. Daremos, a seguir, uma outra prova para $c \in\{-1,1\}$.

Proposição 1.1.15. Seja $h_{s}: M^{n-1} \rightarrow \mathbb{Q}_{c}^{n}$ uma família de hipersuperfícies paralelas a uma hipersuperfície $h: M^{n-1} \rightarrow \mathbb{Q}_{c}^{n}$ dada por $h_{s}(x)=C_{c}(s) h(x)+S_{c}(s) N(x)$, onde $N$ é o vetor unitário normal a $h$. Então as curvaturas principais $\lambda_{i}^{s}(x)$, com $i \in\{1, \ldots, n-1\}$, de $h_{s}$ são dadas por

$$
\lambda_{i}^{s}(x)=\frac{c S_{c}(s)+C_{c}(s) \lambda_{i}(x)}{C_{c}(s)-S_{c}(s) \lambda_{i}(x)}
$$


onde $\lambda_{i}$ é uma curvatura principal de $h$.

Demonstração. Temos que $h_{s}(x)=C_{c}(s) h(x)+S_{c}(s) N(x)$ e seu campo normal unitário é dado por $N_{s}(x)=-c S_{c}(s) h(x)+C_{c}(s) N(x)$. Denotaremos aqui a conexão Riemanniana de $\mathbb{Q}_{c}^{n}$ por $\widetilde{\nabla}$. Assim,

$$
d h_{s}(X)=C_{c}(s) d h(X)+S_{c}(s) \widetilde{\nabla}_{X} N=C_{c}(s) d h(X)-S_{c}(s) d h\left(A_{N} X\right),
$$

ou seja,

$$
d h_{s}(X)=d h\left(C_{c}(s) X-S_{c}(s) A X\right),
$$

para todo $X \in T M^{n-1}$, onde $A$ denota o operador de Weingarten de $h$. Observe que para $h_{s}$ ser uma imersão é necessário que $\left(C_{c}(s) I-S_{c}(s) A\right) X \neq 0$, para todo $X \in T M^{n-1}, X$ não nulo, e para cada $s \in J \subset \mathbb{R}$, onde $I$ é a matriz identidade.

Por um lado, temos que $-d h_{s}\left(A^{s} X\right)=\widetilde{\nabla}_{X} N_{s}=-c S_{c}(s) d h(X)+C_{c}(s) \widetilde{\nabla}_{X} N$, isto é, $d h_{s}\left(A^{s} X\right)=d h\left(c S_{c}(s) X+C_{c}(s) A X\right)$. Por outro lado, usando (1.16), obtemos

$$
d h_{s}\left(A^{s} X\right)=d h\left(C_{c}(s) A^{s} X-S_{c}(s) A \circ A^{s} X\right) .
$$

Logo,

$$
d h\left(c S_{c}(s) X+C_{c}(s) A X\right)=\operatorname{dh}\left(C_{c}(s) A^{s} X-S_{c}(s) A \circ A^{s} X\right)
$$

e como $d h$ é injetora, segue que

$$
\left(C_{c}(s) I-S_{c}(s) A\right) \circ A^{s} X=\left(c S_{c}(s) I+C_{c}(s) A\right) X,
$$

para todo $X \in T M^{n-1}$. Considerando $0<s<\pi$ temos $S_{c}(s) \neq 0$ para todo $s$, e dividindo os membros da equação $(1.17)$ por $S_{c}(s) \neq 0$ segue que

$$
A \circ A^{s}=\operatorname{Cot}_{c}(s) A^{s}-c I-\operatorname{Cot}_{c}(s) A,
$$

onde $\operatorname{Cot}_{c}(s)$ denota $\cot (s)$, se $c=1$ ou $\operatorname{coth}(s)$, se $c=-1$.

Uma vez que $A$ e $A^{s}$ são operadores auto-adjuntos, utilizando (1.18) obtemos $\left\langle A \circ A^{s}(X), Y\right\rangle=\left\langle X, A \circ A^{s}(Y)\right\rangle$, para todo $X, Y \in T M^{n-1}$. Além disso,

$$
\left\langle A \circ A^{s}(X), Y\right\rangle=\left\langle A^{s}(X), A Y\right\rangle=\left\langle X, A^{s} \circ A(Y)\right\rangle,
$$

para todo $X, Y \in T M^{n-1}$. Consequentemente $A \circ A^{s}=A^{s} \circ A$. Então, por (1.18), dado $\left\{X_{1}, \ldots, X_{n-1}\right\}$ com $A X_{i}=\lambda_{i} X_{i}$ temos $A^{s} \circ A\left(X_{i}\right)=\operatorname{Cot}_{c}(s) A^{s}\left(X_{i}\right)-\left(c+\operatorname{Cot}_{c}(s) \lambda_{i}\right) X_{i}$, o 
que implica em $A^{s}\left(X_{i}\right)=\frac{c+\operatorname{Cot}_{c}(s) \lambda_{i}}{\operatorname{Cot}_{c}(s)-\lambda_{i}} X_{i}$. Dessa forma, concluimos que

$$
\lambda_{i}^{s}(x)=\frac{c+\operatorname{Cot}_{c}(s) \lambda_{i}(x)}{\operatorname{Cot}_{c}(s)-\lambda_{i}(x)}=\frac{c S_{c}(s)+C_{c}(s) \lambda_{i}(x)}{C_{c}(s)-S_{c}(s) \lambda_{i}(x)} .
$$

Observação 1.1.16. Vimos, pela prova da Proposição 1.1.15, que as direções principais de $h$ também são direções principais de $h_{s}$, para cada $s \in I \subset \mathbb{R}$. Além disso, considerando as curvaturas principais $\lambda_{i}, i \in\{1, \ldots, n-1\}$, de uma hipersuperfície de $\mathbb{S}^{n} \operatorname{como} \lambda_{i}(x)=\cot \left(\theta_{i}(x)\right)$, com $0<\theta_{i}(x)<\pi$, temos que $\lambda_{i}^{s}(x)=\cot \left(\theta_{i}(x)-s\right)$.

Observação 1.1.17. Uma hipersuperfície de $\mathbb{Q}_{c}^{n} \times \mathbb{R}$ dada por (1.15) possui, pela Proposição 1.1.14, a curvatura principal $\lambda$ associada à direção $T$ igual a $\frac{a^{\prime \prime}(s)}{\left(a^{\prime}(s)^{2}+1\right)^{\frac{3}{2}}}$. Então $X(\lambda)=0$, para todo $X \in T M^{n-1}$. Assim, pelas Proposições 1.1.10, e 1.1.15, uma hipersuperfície com $n \geq 3$, dada por (1.15) de $\mathbb{Q}_{c}^{n} \times \mathbb{R}$ é uma hipersuperfície rotacional se $h: M^{n-1} \rightarrow \mathbb{Q}_{c}^{n}$ é umbilica.

\subsection{Hipersuperfícies isoparamétricas em $\mathbb{Q}_{c}^{n}$}

O conteúdo apresentado nesta seção pode ser encontrado com maiores detalhes em [9], [18] e [31].

De acordo com a definição original, uma família de hipersuperfícies $M_{t}$ de uma variedade Riemanniana $\widetilde{M}^{n}$ é isoparamétrica se cada $M_{t}=s^{-1}(t)$, onde $s: \widetilde{M}^{n} \rightarrow \mathbb{R}$ é uma função diferenciável não constante que satisfaz $\|\operatorname{grad} s\|^{2}=F_{1}(s)$ e o Laplaciano, $\Delta s=F_{2}(s)$, para alguma função diferenciável $F_{1}: \mathbb{R} \rightarrow \mathbb{R}$ e alguma $F_{2}: \mathbb{R} \rightarrow \mathbb{R}$. Uma função $s$ nas condições anteriores é chamada de função isoparamétrica.

Cartan provou em [7] que uma condição necessária e suficiente para uma família de hipersuperfícies paralelas em uma variedade Riemanniana ser isoparamétrica é que todas as hipersuperfícies tenham curvatura média constante. Ele também mostrou em [5] que, quando o ambiente é um espaço forma $\mathbb{Q}_{c}^{n}$, a definição de hipersuperfície isoparamétrica é equivalente a todas as curvaturas principais serem constantes. Uma demonstração deste fato pode ser encontrada no Teorema 5.8 de [9].

A seguinte proposição é apresentada em [30] e será utilizada posteriormente.

Proposição 1.2.1 (Proposição 2.2 de [30]). Seja A um tensor simétrico de tipo (1,1) em uma variedade Riemanniana conexa $M^{n}, n \geq 2$, com exatamente dois autovalores distintos em cada ponto. Então as multiplicidades desses autovalores são constantes. 
Considere uma hipersuperfície isoparamétrica $F: M^{n-1} \rightarrow \mathbb{Q}_{c}^{n}$. Denotaremos por $g$ o número de curvaturas principais $\lambda_{1}, \ldots, \lambda_{g}$ distintas de $F$ com respectivas multiplicidades $m_{1}, \ldots, m_{g}$. Veremos no próximo resultado, que as curvaturas principais possuem multiplicidades constantes.

Proposição 1.2.2. Seja $F: M^{n-1} \rightarrow \mathbb{S}^{n}, n \geq 4$, uma hipersuperfície isoparamétrica com $g \geq 3$. Então as multiplicidades das curvaturas principais são constantes.

Demonstração. Seja $m_{i}(x)$ a multiplicidade da curvatura principal constante $\lambda_{i}$ em $x \in$ $M^{n-1}$. Considere os polinômios

$$
\begin{gathered}
p_{0}(x):=\sum_{i=1}^{g} m_{i}(x)=n-1 \quad \mathrm{e} \\
p_{j}(x):=\sum_{i=1}^{g} m_{i}(x) \lambda_{i}^{j}=\operatorname{traço~} A^{j},
\end{gathered}
$$

com $j \in\{1, \ldots, g-1\}$, onde $A$ é o operador de Weingarten de $F, \lambda_{i}^{j}$ e $A^{j}$ denotam a $j$-ésima potência de $\lambda_{i}$ e $A$, respectivamente. Observe que, para cada $j$ a função $p_{j}(x)$ é constante. De fato, como $F$ é isoparamétrica, sua família de paralelas possui curvatura média constante. Portanto podemos considerar a função

$$
u(t)=\sum_{i=1}^{g} m_{i}(x) \cot \left(\theta_{i}-t\right)
$$

onde $\lambda_{i}=\cot \left(\theta_{i}\right)$ e pela Observação 1.1.16, as curvaturas principais das hipersuperfícies paralelas são $\lambda_{i}^{t}=\cot \left(\theta_{i}-t\right)$. Então

$$
u(0)=\sum_{i=1}^{g} m_{i}(x) \cot \left(\theta_{i}\right)=k_{1}
$$

onde $k_{1}$ é uma constante. Fazendo a primeira derivada de $u$ no ponto 0 , obtemos

$$
u^{\prime}(0)=\sum_{i=1}^{g} m_{i}(x)+\sum_{i=1}^{g} m_{i}(x) \cot \left(\theta_{i}\right)^{2}=(n-1)+\sum_{i=1}^{g} m_{i}(x) \cot \left(\theta_{i}\right)^{2},
$$

o que implica em $\sum_{i=1}^{g} m_{i}(x) \cot \left(\theta_{i}\right)^{2}=k_{2}$, onde $k_{2}$ é uma constante. Fazendo a segunda 
derivada de $u$ no ponto 0 , temos

$$
u^{\prime \prime}(0)=2 \sum_{i=1}^{g} m_{i}(x) \cot \left(\theta_{i}\right)+2 \sum_{i=1}^{g} m_{i}(x) \cot \left(\theta_{i}\right)^{3}=2 k_{1}+2 \sum_{i=1}^{g} m_{i}(x) \cot \left(\theta_{i}\right)^{3}
$$

e portanto $\sum_{i=1}^{g} m_{i}(x) \cot \left(\theta_{i}\right)^{3}=k_{3}$, onde $k_{3}$ é uma constante.

Continuando esse processo até a $(g-2)$-derivada de $u$ no ponto 0 concluimos que $\sum_{i=1}^{g} m_{i}(x) \cot \left(\theta_{i}\right)^{j}$ é constante, $j \in\{1, \ldots, g-1\}$. Logo a função $p_{j}(x)$ é constante para cada $j$. Assim, obtemos um sistema linear não homogêneo de $g$ incógnitas $m_{i}(x)$ e $g$ equações onde a matriz $\left(u_{r s}\right)$ dos coeficientes possui os elementos $u_{1 s}=1$, com $s \in\{1, \ldots, g\}$. Utilizando a regra de Cramer, concluimos que as multiplicidades são constantes.

Para $g>1$, Cartan mostrou que para cada $i \in\{1, \ldots, g\}$,

$$
\sum_{j \neq i} m_{j} \frac{c+\lambda_{i} \lambda_{j}}{\lambda_{i}-\lambda_{j}}=0
$$

Esta equação é conhecida como identidade de Cartan e através dela Cartan classificou as hipersuperfícies isoparamétricas nos casos $c=0$ e $c=-1$, como se pode ver no próximo resultado.

Teorema 1.2.3. (Teorema 5 de [18]) Se $M^{n-1}$ é uma hipersuperfície isoparamétrica de um espaço $\mathbb{Q}_{c}^{n}$ de curvatura constante $c \leq 0$, então o número de curvaturas principais distintas é $g \leq 2$. Para $g=1, M$ é totalmente umbilica. Para $g=2$ as duas curvaturas distintas satisfazem

$$
\lambda_{1} \lambda_{2}=-c
$$

e $M$ é obtida, na vizinhança de um ponto, tomando-se as duas folhas de curvatura (que são subvariedades totalmente umbílicas) que passam por esse ponto e, em seguida, transladando-se paralelamente uma sobre a outra em $\mathbb{Q}_{c}^{n}$.

Dessa forma, as hipersuperfícies isoparamétricas em $\mathbb{R}^{n}$ ou $\mathbb{H}^{n}$ possuem, no máximo, duas curvaturas principais distintas.

No caso $c=0$, se $g=1$ é bem conhecido que $F\left(M^{n-1}\right)$ é um subconjunto aberto de um hiperplano ou uma hiperesfera. Se $g=2, F\left(M^{n-1}\right)$ é um subconjunto aberto de um cilindro esférico.

No caso $c=-1$, se $g=1$, então $F\left(M^{n-1}\right)$ é um subconjunto aberto de um hiperplano totalmente geodésico, uma hipersuperfície equidistante, uma horosfera ou uma hiperesfera 
em $\mathbb{H}^{n}$. Se $g=2$, então $F\left(M^{n-1}\right)$ é um subconjunto aberto de um produto $\mathbb{S}^{k} \times \mathbb{H}^{n-k-1}$.

Para o caso $c>0$, a identidade de Cartan não gerou restrições fortes sobre o número $g$ de curvaturas principais distintas. Cartan produziu exemplos com $g=1,2,3$ ou 4 e classificou todas as hipersuperfícies isoparamétricas $F: M^{n-1} \rightarrow \mathbb{S}^{n}$ com $g \leq 3$. Se $g=1$, a hipersuperfície é totalmente umbílica e portanto uma hiperesfera em $\mathbb{S}^{n}$ formada pela intersecção de $\mathbb{S}^{n}$ com um hiperplano em $\mathbb{R}^{n+1}$. Se $g=2$ então $F\left(M^{n-1}\right)$ é o produto de duas esferas,

$$
\mathbb{S}^{p}(r) \times \mathbb{S}^{q}(s) \subset \mathbb{S}^{n}(1) \subset \mathbb{R}^{p+1} \times \mathbb{R}^{q+1}=\mathbb{R}^{n+1},
$$

com $r^{2}+s^{2}=1$ e $n=p+q+1$. Para $g=3$, Cartan mostrou em [7], que todas curvaturas principais devem ter a mesma multiplicidade $m \in\{1,2,4,8\}$ e $F\left(M^{n-1}\right)$ é um tubo de raio constante sobre um mergulho de um plano projetivo $\mathbb{F P}^{2}$ em $\mathbb{S}^{3 m+1}$, onde $\mathbb{F}$ denota o espaço euclidiano $\mathbb{R}$, o espaço complexo $\mathbb{C}$, os quatérnios $\mathbb{H}$ ou os números de Cayley $\mathbb{O}$, para $m=1,2,4,8$, respectivamente. Em particular, para $\mathbb{F}=\mathbb{R}$ o mergulho é uma superfície de Veronese em $\mathbb{S}^{4}$. A menos de congruência existe somente uma tal família de hipersuperfícies isoparamétricas para cada valor de $m$. Essas hipersuperfícies são chamadas de hipersuperfícies de Cartan.

Como dito na introdução de [14], uma hipersuperfície de Cartan na esfera $\mathbb{S}^{n}(1)$ é uma hipersuperfície compacta com curvaturas principais iguais a $-\sqrt{3}, 0$ e $\sqrt{3}$. Logo, toda hipersuperfície de Cartan é mínima. Além disso, essas hipersuperfícies só existem em $\mathbb{S}^{4}, \mathbb{S}^{7}, \mathbb{S}^{13}$ ou $\mathbb{S}^{25}$.

Em [24] e [25], Münzner estendeu o trabalho de Cartan, mostrando que o número $g$ de curvaturas principais distintas das hipersuperfícies isoparamétricas de $\mathbb{S}^{n}$ deve ser $1,2,3,4$ ou 6. Uma vez que Cartan tinha classificado as hipersuperfícies isoparamétricas de $\mathbb{S}^{n}$ com $g \leq 3$, a classificação dessas hipersuperfícies com $g=4$ e $g=6$ passou a interessar vários pesquisadores da área, como por exemplo, Abresch em [1]. Este problema de classificação tem provado ser interessante e bastante difícil. Ele foi listado como o Problema 34 na lista de Yau [35], em 1990, composta por importantes problemas de geometria em aberto.

Muitos progressos foram feitos no estudo de hipersuperfícies isoparamétricas de $\mathbb{S}^{n}$ com $g=4$ e $g=6$. Dentre eles, destacamos os trabalhos [10], [11], [12] de T. Cecil, Q-S. Chi e G. Jensen para $g=4$ e mais recentemente, o trabalho [21] de R. Miyaoka que completa a classificação para o caso $g=6$. 


\section{Capítulo 2}

\section{Família de hipersuperfícies paralelas em $\mathbb{Q}_{c}^{n} \times \mathbb{R}$}

Neste capítulo vamos estudar uma família de hipersuperfícies paralelas de uma imersão isométrica $f: M^{n} \rightarrow \mathbb{Q}_{c}^{n} \times \mathbb{R}$. Nosso objetivo é mostrar que quando o ambiente é $\mathbb{Q}_{c}^{n} \times \mathbb{R}$, a definição clássica de hipersuperfícies isoparamétricas pode não ser equivalente às curvaturas principais serem constantes. De fato, a equivalência ocorre com hipóteses adicionais, que veremos no decorrer do capítulo.

\subsection{Existência de um referencial local de direções prin- cipais}

Nesta seção apresentamos o seguinte teorema, baseado em resultados encontrados em [13] e [27], que é de grande importância pois garante a existência de um referencial local de direções principais diferenciáveis.

Teorema 2.1.1. Seja A um tensor simétrico do tipo $(1,1)$ em uma variedade Riemanianna orientada $M^{n}, n \geq 2$, com $g$ autovalores distintos $\lambda_{1}, \ldots, \lambda_{g}$ de multiplicidades constantes $m_{1}, \ldots, m_{g}$, respectivamente. Então, para cada ponto $p \in M$, existe um referencial ortonormal de autovetores diferenciáveis $\left\{X_{1}, \ldots, X_{n}\right\}$ em uma vizinhança $U$ de $p$ em $M$.

Demonstração. Sem perda da generalidade, podemos supor $\lambda_{1}>\lambda_{2}>\ldots>\lambda_{g}$. Considere as $g$ distribuições ortogonais $D_{\lambda_{i}}$, com $i \in\{1, \ldots, g\}$ definidas por

$$
D_{\lambda_{i}}(p)=\left\{Y_{p} \in T_{p} M ; A Y_{p}=\lambda_{i} Y_{p}\right\}
$$

Dado $p \in M$, seja $U^{\prime}$ uma vizinhança de $p$ em $M$ onde estão definidos os campos diferenciáveis 
$Y_{1}, \ldots, Y_{n}$ tais que $\left\{Y_{1}^{1}(p), \ldots, Y_{m_{1}}^{1}(p)\right\}$ gera a distribuição $D_{\lambda_{1}}(p),\left\{Y_{m_{1}+1}^{2}(p), \ldots, Y_{m_{1}+m_{2}}^{2}(p)\right\}$ gera a distribuição $D_{\lambda_{2}}(p), \ldots,\left\{Y_{m_{1}+\ldots+m_{g-1}+1}^{g}(p), \ldots, Y_{n}^{g}(p)\right\}$ gera a distribuição $D_{\lambda_{g}}(p)$.

Defina campos $X_{1}, \ldots, X_{n}$ em $U^{\prime}$ por

$$
\begin{gathered}
X_{i}^{1}(x)=\left(A(x)-\lambda_{2} I\right) \ldots\left(A(x)-\lambda_{g} I\right) Y_{i}^{1}(x), \text { para } i \in\left\{1, \ldots, m_{1}\right\}, \\
X_{i}^{2}(x)=-\left(A(x)-\lambda_{1} I\right)\left(A(x)-\lambda_{3} I\right) \ldots\left(A(x)-\lambda_{g} I\right) Y_{i}^{2}(x), \text { para } i \in\left\{m_{1}+1, \ldots, m_{1}+m_{2}\right\}
\end{gathered}
$$

e

$$
X_{i}^{k}(x)=(-1)^{k-1} \prod_{j \neq k}\left(A(x)-\lambda_{j} I\right) Y_{i}^{k}(x),
$$

para cada $k \in\{3, \ldots, g\}$ e $i \in\left\{m_{1}+\ldots+m_{k-1}+1, \ldots, m_{1}+\ldots+m_{k}\right\}$, com $j \in\{1, \ldots, g\}$, onde $I$ é a matriz identidade de ordem $n$ e $x \in U^{\prime}$.

Observe que esses campos dependem diferenciavelmente de $x$, pois como os autovalores possuem multiplicidades constantes, eles são diferenciáveis [27]. Além disso, uma vez que $\left\{X_{1}(p), \ldots, X_{n}(p)\right\}$ é linearmente independente, temos que $\left\{X_{1}(x), \ldots, X_{n}(x)\right\}$ é linearmente independente para todo $x$ em uma vizinhança $U \subset U^{\prime}$ de $p$ em $M$. Observe também que, para cada $x \in U$, a base $\left\{X_{1}(x), \ldots, X_{n}(x)\right\}$ é positiva pois possui a mesma orientação que a base $\left\{Y_{1}(x), \ldots, Y_{n}(x)\right\}$.

O polinômio característico do operador $A(x)$ é $p(t, x)=\left(t-\lambda_{1}\right)^{m_{1}}\left(t-\lambda_{2}\right)^{m_{2}} \ldots\left(t-\lambda_{g}\right)^{m_{g}} \mathrm{e}$ pelo Teorema de Cayley-Hamilton, temos que $\left(A(x)-\lambda_{1} I\right)\left(A(x)-\lambda_{2} I\right) \ldots\left(A(x)-\lambda_{g} I\right)=0$, para cada $x \in U$. Logo, $\left(A(x)-\lambda_{1} I\right)\left(A(x)-\lambda_{2} I\right) \ldots\left(A(x)-\lambda_{g} I\right) Y_{i}(x)=0$, para todo $i \in$ $\{1, \ldots, n\}$ e $x \in U$. Dessa forma, para todo $x \in U$,

$$
\left(A(x)-\lambda_{1} I\right) X_{i}^{1}(x)=\left(A(x)-\lambda_{1} I\right)\left(A(x)-\lambda_{2} I\right) \ldots\left(A(x)-\lambda_{g} I\right) Y_{i}^{1}(x)=0,
$$

para todo $i \in\left\{1, \ldots m_{1}\right\}$,

$$
\left(A(x)-\lambda_{2} I\right) X_{i}^{2}(x)=-\left(A(x)-\lambda_{1} I\right)\left(A(x)-\lambda_{2} I\right) \ldots\left(A(x)-\lambda_{g} I\right) Y_{i}^{2}(x)=0,
$$

para todo $i \in\left\{m_{1}+1, \ldots, m_{1}+m_{2}\right\}$,

$$
\left(A(x)-\lambda_{k} I\right) X_{i}^{k}(x)=(-1)^{k-1}\left(A(x)-\lambda_{1} I\right)\left(A(x)-\lambda_{2} I\right) \ldots\left(A(x)-\lambda_{g} I\right) Y_{i}^{k}(x)=0,
$$

para todo $i \in\left\{m_{1}+\ldots+m_{k-1}+1, \ldots, m_{1}+\ldots+m_{k}\right\}$. Portanto, para cada $x \in U$, temos que $A(x) X_{i}^{k}(x)=\lambda_{k} X_{i}^{k}(x)$, para todo $i \in\{1, \ldots, n\}$ e $k \in\{1, \ldots, g\}$.

Pelo processo de ortogonalização de Gram-Schmidt obtemos os conjuntos ortonormaliza$\operatorname{dos}\left\{X_{1}^{1}(x), \ldots, X_{m_{1}}^{1}(x)\right\},\left\{X_{m_{1}+1}^{2}(x), \ldots, X_{m_{1}+m_{2}}^{2}(x)\right\}$ e $\left\{X_{m_{1}+\ldots+m_{k-1}+1}^{k}(x), \ldots, X_{m_{1}+\ldots+m_{k}}^{k}(x)\right\}$, 
para cada $x \in U$ e $k \in\{3, \ldots, g\}$. Além disso, sejam $X_{i}^{\alpha}$ e $X_{j}^{\beta} \operatorname{com} i, j \in\{1, \ldots, n\}$ e $\alpha, \beta \in\{1, \ldots, g\}$ com $\alpha \neq \beta$. Então,

$$
\begin{aligned}
\left(\lambda_{\alpha}-\lambda_{\beta}\right)\left\langle X_{i}^{\alpha}, X_{j}^{\beta}\right\rangle & =\left\langle\lambda_{\alpha} X_{i}^{\alpha}, X_{j}^{\beta}\right\rangle-\left\langle X_{i}^{\alpha}, \lambda_{\beta} X_{j}^{\beta}\right\rangle=\left\langle A X_{i}^{\alpha}, X_{j}^{\beta}\right\rangle-\left\langle X_{i}^{\alpha}, A X_{j}^{\beta}\right\rangle \\
& =\left\langle A X_{i}^{\alpha}-A X_{i}^{\alpha}, X_{j}^{\beta}\right\rangle=0 .
\end{aligned}
$$

Ou seja, $\left\langle X_{i}^{\alpha}, X_{j}^{\beta}\right\rangle=0$, para $i, j \in\{1, \ldots, n\}$ e $\alpha, \beta \in\{1, \ldots, g\}$ com $\alpha \neq \beta$. Obtemos assim um referencial ortonormal local de autovetores diferenciáveis $\left\{X_{1}, \ldots, X_{n}\right\}$.

Portanto o resultado acima vale para hipersuperfícies em $\mathbb{Q}_{c}^{n} \times \mathbb{R}$, com o tensor simétrico sendo o operador de Weingarten e supondo que as multiplicidades das curvaturas principais são constantes.

Corolário 2.1.2. Seja $f: M^{n} \rightarrow \mathbb{Q}_{c}^{n} \times \mathbb{R}, n \geq 2$, uma hipersuperfície com $g \geq 2$ curvaturas principais distintas $\lambda_{1}, \ldots, \lambda_{g}$, com multiplicidades constantes $m_{1}, \ldots, m_{g}$, respectivamente. Então, para cada ponto $p \in M$, existe um referencial ortonormal de direções principais $\left\{X_{1}, \ldots, X_{n}\right\}$ em uma vizinhança $U$ de $p$ em $M$.

\subsection{Construção da família de paralelas}

Considere as hipersuperfícies $f: M^{n} \rightarrow \mathbb{Q}_{c}^{n} \times \mathbb{R}$ e $i: \mathbb{Q}_{c}^{n} \times \mathbb{R} \rightarrow \mathbb{E}^{n+2}$, com campos normais $\eta$ e $\xi$, respectivamente, sendo $\eta$ unitário e $\langle\xi, \xi\rangle=c$. Sejam $F:=i \circ f, \bar{\nabla}$ e $\widehat{\nabla}$ as conexões Riemannianas de $\mathbb{Q}_{c}^{n} \times \mathbb{R}$ e $\mathbb{E}^{n+2}$, respectivamente.

Sejam $\pi_{1}: \mathbb{Q}_{c}^{n} \times \mathbb{R} \rightarrow \mathbb{Q}_{c}^{n}$ e $\pi_{2}: \mathbb{Q}_{c}^{n} \times \mathbb{R} \rightarrow \mathbb{R}$ as projeções canônicas. Dados $t \in \mathbb{R}, p \in M^{n}$ e $v \in T_{f(p)}\left(\mathbb{Q}_{c}^{n} \times \mathbb{R}\right)$ com $d_{f(p)} \pi_{1}(v)=v_{1}$ e $d_{f(p)} \pi_{2}(v)=v_{2}$, a aplicação exponencial em $\mathbb{Q}_{c}^{n} \times \mathbb{R}$ é dada por

$$
\exp _{f(p)}(t v)=\left(C_{c}\left(\left\|v_{1}\right\| t\right) \pi_{1}(f(p))+S_{c}\left(\left\|v_{1}\right\| t\right) \frac{v_{1}}{\left\|v_{1}\right\|}, \pi_{2}(f(p))+t v_{2}\right)
$$

se $v_{1} \neq 0$, e

$$
\exp _{f(p)}(t v)=\left(\pi_{1}(f(p)), \pi_{2}(f(p))+t v_{2}\right), \quad \text { se } \quad v_{1}=0 .
$$

Lembramos que $C_{c}$ e $S_{c}$ foram definidos em (1.14). Dados $p \in M^{n}$ e $v \in T_{f(p)}\left(\mathbb{Q}_{c}^{n} \times \mathbb{R}\right)$, considere a curva $\alpha: I \subset \mathbb{R} \rightarrow \mathbb{Q}_{c}^{n} \times \mathbb{R}$ dada por $\alpha(t)=\exp _{f(p)}(t v)$. Observe que $\alpha$ é uma geodésica em $\mathbb{Q}_{c}^{n} \times \mathbb{R}$ que passa pelo ponto $\alpha(0)=\left(\pi_{1}(f(p)), \pi_{2}(f(p))\right)=f(p)$ e $\alpha^{\prime}(0)=\left(v_{1}, v_{2}\right)=v$.

As duas proposições seguintes fornecem equações que serão utilizadas no decorrer deste capítulo. 
Proposição 2.2.1. Valem as seguintes igualdades, para todo $X \in T M^{n}$,

$$
\begin{gathered}
\widehat{\nabla}_{X} \xi=d f(X)-\langle X, T\rangle \partial / \partial t \\
\nabla_{X}^{\perp} \xi=-\nu\langle X, T\rangle \eta \\
\nabla_{X}^{\perp} \eta=c \nu\langle X, T\rangle \xi
\end{gathered}
$$

Demonstração. Dado um ponto $q=\left(q_{1}, q_{2}, \ldots, q_{n+1}, q_{n+2}\right) \in \mathbb{Q}_{c}^{n} \times \mathbb{R} \subset \mathbb{E}^{n+2}$, temos que $\xi(q)=\left(q_{1}, q_{2}, \ldots, q_{n+1}, 0\right)$. Assim, para todo $X \in T M^{n}, \widehat{\nabla}_{X} \xi=X_{\mathbb{Q}}=d f(X)-\langle X, T\rangle \partial / \partial t$. Além disso,

$$
\nabla_{X}^{\perp} \xi=\left\langle\widehat{\nabla}_{X} \xi, \eta\right\rangle \eta+c\left\langle\widehat{\nabla}_{X} \xi, \xi\right\rangle \xi=-\nu\langle X, T\rangle \eta
$$

e $\nabla_{X}^{\perp} \eta=\left\langle\widehat{\nabla}_{X} \eta, \eta\right\rangle \eta+c\left\langle\widehat{\nabla}_{X} \eta, \xi\right\rangle \xi=-c\left\langle\eta, \widehat{\nabla}_{X} \xi\right\rangle \xi$.

Proposição 2.2.2. Suponha que as curvaturas principais de $f: M^{n} \rightarrow \mathbb{Q}_{c}^{n} \times \mathbb{R}$ possuem multiplicidades constantes. Sejam $\left\{X_{1}, \ldots, X_{n}\right\}$ um referencial de direções principais ortonormais e $\lambda_{i}$ a curvatura principal associada a $X_{i}$. Se $T$ é uma direção principal, $X_{n}=\|T\|^{-1} T e$ $\eta_{\mathbb{Q}}=\eta-\nu \partial / \partial t$, ent $\tilde{a} O$

$$
\begin{gathered}
\widehat{\nabla}_{X_{i}} \eta_{\mathbb{Q}}=-\lambda_{i} d f\left(X_{i}\right)+c \nu\left\langle X_{i}, T\right\rangle \xi-X_{i}(\nu) \partial / \partial t, \\
X_{i}(\|T\|)=0, \quad \text { para todo } i \neq n, \quad \text { e } \quad X_{n}(\|T\|)=\nu \lambda_{n}, \\
X_{i}(\nu)=0, \quad \text { para todo } i \neq n, \quad \text { e } \quad X_{n}(\nu)=-\lambda_{n}\|T\|, \\
X_{i}\left(\pi_{2} \circ f\right)=0, \quad \text { para todo } i \neq n \quad \text { e } \quad X_{n}\left(\pi_{2} \circ f\right)=\|T\| .
\end{gathered}
$$

Demonstração. Observe que

$$
\widehat{\nabla}_{X_{i}} \eta_{\mathbb{Q}}=\widehat{\nabla}_{X_{i}} \eta-X_{i}(\nu) \partial / \partial t=-d f\left(A_{\eta} X_{i}\right)+\nabla_{X_{i}}^{\perp} \eta-X_{i}(\nu) \partial / \partial t
$$

Assim, por (2.3), obtemos $\widehat{\nabla}_{X_{i}} \eta_{\mathbb{Q}}=-\lambda_{i} d f\left(X_{i}\right)+c \nu\left\langle X_{i}, T\right\rangle \xi-X_{i}(\nu) \partial / \partial t$.

Utilizando (1.5) e (1.6), temos para todo $i \in\{1, \ldots, n\}$,

$$
2\|T\| X_{i}(\|T\|)=X_{i}\left(\|T\|^{2}\right)=X_{i}\langle T, T\rangle=2\left\langle\nabla_{X_{i}} T, T\right\rangle=2 \nu \lambda_{i}\left\langle X_{i}, T\right\rangle
$$

e $X_{i}(\nu)=-\left\langle A_{\eta} X_{i}, T\right\rangle=-\left\langle X_{i}, A_{\eta} T\right\rangle=-\lambda_{n}\left\langle X_{i}, T\right\rangle$. Além disso,

$$
X_{i}\left(\pi_{2} \circ f\right)=d \pi_{2}\left(d f\left(X_{i}\right)\right)=\pi_{2} d f\left(X_{i}\right)=\left\langle d f\left(X_{i}\right), \partial / \partial t\right\rangle=\left\langle X_{i}, T\right\rangle .
$$


Estudaremos a família de hipersuperfícies paralelas a uma hipersuperfície que possui $T$ como uma direção principal.

Seja $f: M^{n} \rightarrow \mathbb{Q}_{c}^{n} \times \mathbb{R}$ uma hipersuperfície que possui $T$ como uma direção principal e com curvaturas principais de multiplicidades constantes. Seja $\left\{X_{1}, \ldots, X_{n}\right\}$ um referencial de direções principais ortonormais. Suponha que $X_{n}=\|T\|^{-1} T$. Observe que $\xi \circ f=\left(\pi_{1} \circ f, 0\right)$ e $\eta_{\mathbb{Q}}=\eta-\nu \partial / \partial t$, o que implica em $\left\|\eta_{\mathbb{Q}}\right\|=\|T\| \neq 0$. Então as hipersuperfícies paralelas a $f$ são

$$
f_{t}=C_{c}(\|T\| t) \xi \circ f+S_{c}(\|T\| t)\|T\|^{-1} \eta_{\mathbb{Q}}+\left(\pi_{2} \circ f+t \nu\right) \partial / \partial t .
$$

Temos, para todo $i \in\{1, \ldots, n\}$, que

$$
\begin{aligned}
d f_{t}\left(X_{i}\right)= & -c t S_{c}(\|T\| t) X_{i}(\|T\|) \xi+C_{c}(\|T\| t) \widehat{\nabla}_{X_{i}} \xi+t C_{c}(\|T\| t) X_{i}(\|T\|)\|T\|^{-1} \eta_{\mathbb{Q}}+ \\
& +S_{c}(\|T\| t) \widehat{\nabla}_{X_{i}}\|T\|^{-1} \eta_{\mathbb{Q}}+X_{i}\left(\pi_{2} \circ f+t \nu\right) \partial / \partial t
\end{aligned}
$$

Então, por (2.1), (2.4), (2.5) e (2.7), obtemos

$$
\begin{aligned}
& d f_{t}\left(X_{i}\right)=\left(C_{c}(\|T\| t)-\lambda_{i}\|T\|^{-1} S_{c}(\|T\| t)\right) d f\left(X_{i}\right), \quad \text { para } i \neq n \quad \text { e } \\
& d f_{t}\left(X_{n}\right)= \\
& c \nu S_{c}(\|T\| t)\left(1-t \lambda_{n}\right) \xi+\left(1-t \lambda_{n}\right)\left(\nu^{2} C_{c}(\|T\| t)+\|T\|^{2}\right) d f\left(X_{n}\right)+ \\
& +\left(1-t \lambda_{n}\right)\left(1-C_{c}(\|T\| t)\right) \nu\|T\| \eta .
\end{aligned}
$$

Assim, para $f_{t}$ ser uma imersão é necessário que $C_{c}(\|T\| t)-\lambda_{i}\|T\|^{-1} S_{c}(\|T\| t) \neq 0$, para todo $i \in\{1, \ldots, n-1\}$ e $1-t \lambda_{n} \neq 0$.

Observe que o campo unitário normal a $f_{t}$ é dado por

$$
\eta_{t}=-c\|T\| S_{c}(\|T\| t) \xi \circ f+C_{c}(\|T\| t) \eta_{\mathbb{Q}}+\nu \partial / \partial t
$$

De fato,

$$
\begin{gathered}
\left\langle\eta_{t}, \eta_{t}\right\rangle=c\|T\|^{2} S_{c}^{2}(\|T\| t)+\|T\|^{2} C_{c}^{2}(\|T\| t)+\nu^{2}=\|T\|^{2}+\nu^{2}=1, \\
\left\langle\eta_{t}, \xi \circ f_{t}\right\rangle=\left\langle\eta_{t}, C_{c}(\|T\| t) \xi \circ f+S_{c}(\|T\| t)\|T\|^{-1} \eta_{\mathbb{Q}}\right\rangle=0,
\end{gathered}
$$

$\left\langle\eta_{t}, d f_{t}\left(X_{i}\right)\right\rangle=0$, para $i \neq n$. Além disso, observe que $\partial / \partial t=d f(T)+\nu \eta=\|T\| d f\left(X_{n}\right)+\nu \eta$ e

$$
\eta_{\mathbb{Q}}=\eta-\nu \partial / \partial t=\eta-\nu\|T\| d f\left(X_{n}\right)-\nu^{2} \eta=\|T\|^{2} \eta-\nu\|T\| d f\left(X_{n}\right)
$$

Portanto, podemos reescrever $\eta_{t}$ como

$$
\eta_{t}=-c\|T\| S_{c}(\|T\| t) \xi \circ f+\left(\|T\|^{2} C_{c}(\|T\| t)+\nu^{2}\right) \eta+\left(1-C_{c}(\|T\| t)\right) \nu\|T\| d f\left(X_{n}\right)
$$


e então

$$
\begin{aligned}
\left\langle\eta_{t}, d f_{t}\left(X_{n}\right)\right\rangle= & -c \nu\|T\|\left(1-t \lambda_{n}\right) S_{c}^{2}(\|T\| t)+ \\
& +\nu\|T\|\left(1-t \lambda_{n}\right)\left(1-C_{c}(\|T\| t)\right)\left(\|T\|^{2} C_{c}(\|T\| t)+\nu^{2}+\nu^{2} C_{c}(\|T\| t)+\|T\|^{2}\right) \\
= & -c \nu\|T\|\left(1-t \lambda_{n}\right) S_{c}^{2}(\|T\| t)+\nu\|T\|\left(1-t \lambda_{n}\right)\left(1-C_{c}(\|T\| t)\right)\left(1+C_{c}(\|T\| t)\right) \\
= & \nu\|T\|\left(1-t \lambda_{n}\right)\left(-c S_{c}^{2}(\|T\| t)+1-C_{c}^{2}(\|T\| t)\right) \\
= & 0 .
\end{aligned}
$$

\subsection{Relação entre as curvaturas principais de uma hiper- superfície e sua família de paralelas}

Nesta seção calculamos as curvaturas principais das hipersuperfícies paralelas $f_{t}$. Para isso, precisamos de direções principais em $f_{t}$.

Proposição 2.3.1. Se $\left\{X_{1}, \ldots, X_{n}\right\}$ é um referencial de direções principais ortogonais em $f$, então ele é também um referencial de direções principais ortogonais em $f_{t}$.

Demonstração. Observe por (2.9) e (2.10) que $\left\langle d f_{t}\left(X_{i}\right), d f_{t}\left(X_{j}\right)\right\rangle=0$, para todo $i \neq j$ com $i, j \in\{1, \ldots, n\}$. Vamos calcular $\widehat{\nabla}_{X_{i}} \eta_{t}$ e mostrar que $\left\langle\widehat{\nabla}_{X_{i}} \eta_{t}, d f_{t}\left(X_{j}\right)\right\rangle=0$, para todo $i \neq j$.

Temos, por (2.11), que

$$
\begin{aligned}
\widehat{\nabla}_{X_{i}} \eta_{t}= & -c X_{i}\left(\|T\| S_{c}(\|T\| t)\right) \xi-c\|T\| S_{c}(\|T\| t) \widehat{\nabla}_{X_{i}} \xi+X_{i}\left(C_{c}(\|T\| t)\right) \eta_{\mathbb{Q}}+C_{c}(\|T\| t) \widehat{\nabla}_{X_{i}} \eta_{\mathbb{Q}}+ \\
& +X_{i}(\nu) \partial / \partial t
\end{aligned}
$$

onde

$$
X_{i}\left(\|T\| S_{c}(\|T\| t)\right)=\left(S_{c}(\|T\| t)+t\|T\| C_{c}(\|T\| t)\right) X_{i}(\|T\|)
$$

e

$$
X_{i}\left(C_{c}(\|T\| t)\right)=-c t S_{c}(\|T\| t) X_{i}(\|T\|) .
$$

Então, pelas equações (2.1), (2.4), (2.5) e (2.6), obtemos

$$
\begin{aligned}
& \widehat{\nabla}_{X_{i}} \eta_{t}=-\left(c\|T\| S_{c}(\|T\| t)+\lambda_{i} C_{c}(\|T\| t)\right) d f\left(X_{i}\right), \quad \text { para } \quad i \neq n \quad \text { e } \\
& \widehat{\nabla}_{X_{n}} \eta_{t}=\left\{\left(1-t \lambda_{n}\right)\|T\| C_{c}(\|T\| t)-\lambda_{n} S_{c}(\|T\| t)\right\} c \nu \xi+ \\
&+\left\{-\lambda_{n}\left(\|T\|^{2}+\nu^{2} C_{c}(\|T\| t)\right)-c \nu^{2}\|T\| S_{c}(\|T\| t)\left(1-t \lambda_{n}\right)\right\} d f\left(X_{n}\right)+ \\
&+\left\{c \nu\|T\|^{2} S_{c}(\|T\| t)\left(1-t \lambda_{n}\right)-\nu\|T\| \lambda_{n}\left(1-C_{c}(\|T\| t)\right)\right\} \eta .
\end{aligned}
$$

Logo, por $(2.9),(2.10),(2.12)$ e $(2.13)$ temos que $\left\langle\widehat{\nabla}_{X_{i}} \eta_{t}, d f_{t}\left(X_{j}\right)\right\rangle=0$, para todo $i \neq j$ com $i, j \in\{1, \ldots, n\}$ e consequentemente $\left\{X_{1}, \ldots, X_{n}\right\}$ é um referencial de direções principais ortogonais em $f_{t}$. 
A seguir, apresentamos a relação entre as curvaturas principais de uma hipersuperfície em $\mathbb{Q}_{c}^{n} \times \mathbb{R}$ que possui $T$ como uma direção principal e de suas hipersuperfícies paralelas.

Proposição 2.3.2. Seja $f: M^{n} \rightarrow \mathbb{Q}_{c}^{n} \times \mathbb{R}$ uma hipersuperfície que possui $T$ como uma direção principal e $\lambda_{i}, i \in\{1, \ldots, n\}$, suas curvaturas principais. Se $f_{t}$ é uma família de hipersuperfícies paralelas à $f$ com curvaturas principais $\lambda_{i}^{t}, i \in\{1, \ldots, n\}$, então

$$
\begin{gathered}
\lambda_{i}^{t}=\frac{c\|T\| S_{c}(\|T\| t)+\lambda_{i} C_{c}(\|T\| t)}{C_{c}(\|T\| t)-\lambda_{i}\|T\|^{-1} S_{c}(\|T\| t)}, \quad i \neq n, \\
e \quad \lambda_{n}^{t}=\frac{\lambda_{n}}{1-t \lambda_{n}} .
\end{gathered}
$$

Demonstração. Note, por (2.9) e (2.10), que

$$
\begin{gathered}
\left\langle d f_{t}\left(X_{i}\right), d f_{t}\left(X_{i}\right)\right\rangle=\left(C_{c}(\|T\| t)-\lambda_{i}\|T\|^{-1} S_{c}(\|T\| t)\right)^{2}, \quad \text { para } i \neq n \quad \text { e } \\
\left\langle d f_{t}\left(X_{n}\right), d f_{t}\left(X_{n}\right)\right\rangle=\left(1-t \lambda_{n}\right)^{2} .
\end{gathered}
$$

Além disso, utilizando (2.9) e (2.12) obtemos, para $i \neq n$,

$$
-\left\langle\widehat{\nabla}_{X_{i}} \eta_{t}, d f_{t}\left(X_{i}\right)\right\rangle=\left(c\|T\| S_{c}(\|T\| t)+\lambda_{i} C_{c}(\|T\| t)\right)\left(C_{c}(\|T\| t)-\lambda_{i}\|T\|^{-1} S_{c}(\|T\| t)\right)
$$

e por (2.10) e (2.13) concluimos que

$$
\begin{aligned}
\left\langle\widehat{\nabla}_{X_{n}} \eta_{t}, d f_{t}\left(X_{n}\right)\right\rangle= & c \nu^{2} S_{c}(\|T\| t)\left(1-t \lambda_{n}\right)\left\{\left(1-t \lambda_{n}\right)\|T\| C_{c}(\|T\| t)-\lambda_{n} S_{c}(\|T\| t)\right\}+ \\
& +\left(1-t \lambda_{n}\right)\left(\nu^{2} C_{c}(\|T\| t)+\|T\|^{2}\right)\left\{-\lambda_{n}\left(\|T\|^{2}+\nu^{2} C_{c}(\|T\| t)\right)+\right. \\
& \left.-c \nu^{2}\|T\| S_{c}(\|T\| t)\left(1-t \lambda_{n}\right)\right\}+ \\
& +\nu\|T\|\left(1-t \lambda_{n}\right)\left(1-C_{c}(\|T\| t)\right)\left\{c \nu\|T\|^{2} S_{c}(\|T\| t)\left(1-t \lambda_{n}\right)+\right. \\
& \left.-\nu\|T\| \lambda_{n}\left(1-C_{c}(\|T\| t)\right)\right\} \\
= & \left(1-t \lambda_{n}\right)\left\{c \nu^{2}\|T\| S_{c}(\|T\| t) C_{c}(\|T\| t)\left(1-t \lambda_{n}\right)-c \nu^{2} \lambda_{n} S_{c}^{2}(\|T\| t)+\right. \\
& -\lambda_{n}\left(\nu^{2} C_{c}(\|T\| t)+\|T\|^{2}\right)^{2}-c \nu^{2}\|T\| S_{c}(\|T\| t)\left(1-t \lambda_{n}\right) \nu^{2} C_{c}(\|T\| t)+ \\
& \left.-c \nu^{2}\|T\|^{3}\left(1-t \lambda_{n}\right) S_{c}(\|T\| t) C_{c}(\|T\| t)-\nu^{2}\|T\|^{2} \lambda_{n}\left(1-C_{c}(\|T\| t)\right)^{2}\right\} \\
= & -\lambda_{n}\left(1-t \lambda_{n}\right)\left\{c \nu^{2} S_{c}^{2}(\|T\| t)+\nu^{4} C_{c}^{2}(\|T\| t)+2 \nu^{2}\|T\|^{2} C_{c}(\|T\| t)+\right. \\
& \left.+\|T\|^{4}+\nu^{2}\|T\|^{2}\left(1-2 C_{c}(\|T\| t)+C_{c}^{2}(\|T\| t)\right)\right\} \\
= & -\lambda_{n}\left(1-t \lambda_{n}\right),
\end{aligned}
$$

ou seja,

$$
\left\langle\widehat{\nabla}_{X_{n}} \eta_{t}, d f_{t}\left(X_{n}\right)\right\rangle=-\lambda_{n}\left(1-t \lambda_{n}\right)
$$


Agora, por (2.16) e (2.18), podemos calcular

$$
\lambda_{i}^{t}=-\frac{\left\langle\widehat{\nabla}_{X_{i}} \eta_{t}, d f_{t}\left(X_{i}\right)\right\rangle}{\left\langle d f_{t}\left(X_{i}\right), d f_{t}\left(X_{i}\right)\right\rangle}=\frac{\left(c\|T\| S_{c}(\|T\| t)+\lambda_{i} C_{c}(\|T\| t)\right)\left(C_{c}(\|T\| t)-\lambda_{i}\|T\|^{-1} S_{c}(\|T\| t)\right)}{\left(C_{c}(\|T\| t)-\lambda_{i}\|T\|^{-1} S_{c}(\|T\| t)\right)^{2}}
$$

para $i \neq n$, ou seja,

$$
\lambda_{i}^{t}=\frac{c\|T\| S_{c}(\|T\| t)+\lambda_{i} C_{c}(\|T\| t)}{C_{c}(\|T\| t)-\lambda_{i}\|T\|^{-1} S_{c}(\|T\| t)} .
$$

Para $i=n$, temos, por (2.17) e (2.19) que $\lambda_{n}^{t}=-\frac{\left\langle\widehat{\nabla}_{X_{n}} \eta_{t}, d f_{t}\left(X_{n}\right)\right\rangle}{\left\langle d f_{t}\left(X_{n}\right), d f_{t}\left(X_{n}\right)\right\rangle}=\frac{\lambda_{n}\left(1-t \lambda_{n}\right)}{\left(1-t \lambda_{n}\right)^{2}}$, isto é,

$$
\lambda_{n}^{t}=\frac{\lambda_{n}}{1-t \lambda_{n}}
$$

O próximo resultado mostra que se uma hipersuperfície possui o campo $T$ como uma direção principal então sua família de hipersuperfícies paralelas também o possui.

Proposição 2.3.3. Seja $f: M^{n} \rightarrow \mathbb{Q}_{c}^{n} \times \mathbb{R}$ uma hipersuperfície e $f_{t}$ sua família de hipersuperfícies paralelas. Se $T$ é uma direção principal de $f$, então $T^{t}=\frac{T}{1-t \lambda_{n}}$ com $\partial / \partial t=d f_{t}\left(T^{t}\right)+\nu \eta_{t}$, é uma direção principal de $f_{t}$.

Demonstração. Por (2.11), temos

$$
\partial / \partial t-\nu \eta_{t}=c \nu\|T\| S_{c}(\|T\| t) \xi-\nu C_{c}(\|T\| t) \eta_{\mathbb{Q}}+\left(1-\nu^{2}\right) \partial / \partial t
$$

Utilizando que $\eta_{\mathbb{Q}}=\eta-\nu \partial / \partial t$ e $\partial / \partial t=\|T\| d f\left(X_{n}\right)+\nu \eta$ em (2.20), obtemos

$$
\begin{aligned}
\partial / \partial t-\nu \eta_{t}= & c \nu\|T\| S_{c}(\|T\| t) \xi+\left(\nu^{2} C_{c}(\|T\| t)+\|T\|^{2}\right)\|T\| d f\left(X_{n}\right)+ \\
& +\left(1-C_{c}(\|T\| t)\right) \nu\|T\|^{2} \eta
\end{aligned}
$$

Então, por (2.10), segue que

$$
\partial / \partial t-\nu \eta_{t}=\frac{\|T\|}{\left(1-t \lambda_{n}\right)} d f_{t}\left(X_{n}\right)=d f_{t}\left(\frac{T}{1-t \lambda_{n}}\right)=d f_{t}\left(T^{t}\right)
$$

Uma vez que estamos supondo as curvaturas principais $\lambda_{i}, i \in\{1, \ldots, n\}$, com multiplicidades constantes concluimos que as curvaturas $\lambda_{i}^{t}$ também possuem multiplicidades constantes e portanto, por [27], elas são diferenciáveis. 
Proposição 2.3.4. Seja $f: M^{n} \rightarrow \mathbb{Q}_{c}^{n} \times \mathbb{R}$ uma hipersuperfície $e \lambda_{i}, i \in\{1, \ldots, n\}$, as suas curvaturas principais. Então, para cada $i \in\{1, \ldots, n\}$, as funções $\lambda_{i}$ são constantes se, $e$ somente se, $\sum_{i=1}^{n} \lambda_{i}^{k}, 1 \leq k \leq n$, é constante.

Demonstração. Se as funções $\lambda_{i}$ são constantes, para todo $i \in\{1, \ldots, n\}$, então claramente $\sum_{i=1}^{n} \lambda_{i}^{k}, 1 \leq k \leq n$, é constante.

Para provar a recíproca usaremos a ideia dada na página 273 de [9]. Pela identidade de Newton, os coeficientes do polinômio característico do operador de Weingarten $A$, são polinômios em $\sum_{i=1}^{n} \lambda_{i}^{k}, 1 \leq k \leq n$, que por hipótese, são constantes. Logo, as curvaturas principais, que são as raízes do polinômio característico, são constantes.

O próximo resultado apresenta uma condição para que uma hipersuperfície isoparamétrica $f: M^{n} \rightarrow \mathbb{Q}_{c}^{n} \times \mathbb{R}$ tenha curvaturas principais constantes.

Teorema 2.3.5. Seja $f: M^{n} \rightarrow \mathbb{Q}_{c}^{n} \times \mathbb{R}$ uma hipersuperfície isoparamétrica que possui $T$ como uma direção principal. Então f possui curvaturas principais constantes se, e somente se, $\|T\|$ é constante.

Demonstração. Vimos, na Seção 1.2, que uma hipersuperfície é isoparamétrica se ela e sua família de paralelas possuem curvatura média constante. Dessa forma, podemos definir a função que assume valores reais

$$
u(t)=\sum_{i=1}^{n} \lambda_{i}^{t}
$$

Como estamos supondo que $T$ é uma direção principal, utilizamos a seguir, as expressões dadas por (2.14) e (2.15). Observe que

$$
\frac{\partial \lambda_{i}^{t}}{\partial t}=c\|T\|^{2}+\left(\lambda_{i}^{t}\right)^{2}, \quad \text { para } i \in\{1, \ldots, n-1\}
$$

Suponha que $f$ possua curvaturas principais constantes, então $\sum_{i=1}^{n} \lambda_{i}^{k}, 1 \leq k \leq n$, é constante. Obtemos $u^{\prime}(t)=\sum_{i=1}^{n-1} c\|T\|^{2}+\left(\lambda_{i}^{t}\right)^{2}+\left(\lambda_{n}^{t}\right)^{2}$ e portanto

$$
u^{\prime}(0)=(n-1) c\|T\|^{2}+\sum_{i=1}^{n} \lambda_{i}^{2}
$$

Logo $\|T\|$ é constante. 
Reciprocamente, suponha que $\|T\|$ é constante. Então a função $\nu$ é constante e por (1.6), $\lambda_{n}=0$ e portanto, por $(2.15), \lambda_{n}^{t}=0$. Dessa forma, $u(t)=\sum_{i=1}^{n-1} \lambda_{i}^{t}$ e, consequentemente, a $k$-ésima derivada de $u(t)$ satisfaz $u^{k}(t)=\sum_{i=1}^{n-1} \frac{\partial^{k} \lambda_{i}^{t}}{\partial t^{k}}$. Observe que

$$
\frac{\partial^{2} \lambda_{i}^{t}}{\partial t^{2}}=2 c\|T\|^{2} \lambda_{i}^{t}+2\left(\lambda_{i}^{t}\right)^{3}
$$

Provaremos por indução que, para $2<k<n$ ímpar temos,

$$
\begin{aligned}
\frac{\partial^{k} \lambda_{i}^{t}}{\partial t^{k}}= & u_{k, 0} c^{\frac{k+1}{2}}\|T\|^{k+1}+u_{k, 2} c^{\frac{k-1}{2}}\|T\|^{k-1}\left(\lambda_{i}^{t}\right)^{2}+u_{k, 4} c^{\frac{k-3}{2}}\|T\|^{k-3}\left(\lambda_{i}^{t}\right)^{4}+ \\
& +\ldots+u_{k, k+1}\left(\lambda_{i}^{t}\right)^{k+1}
\end{aligned}
$$

onde denotamos por $u_{k, j}$ o $j$-ésimo coeficiente $u_{j}$ da $k$-ésima derivada de $\lambda_{i}^{t}$.

Para $2 \leq k<n$ par temos

$$
\frac{\partial^{k} \lambda_{i}^{t}}{\partial t^{k}}=u_{k, 1} c^{\frac{k}{2}}\|T\|^{k} \lambda_{i}^{t}+u_{k, 3} c^{\frac{k-2}{2}}\|T\|^{k-2}\left(\lambda_{i}^{t}\right)^{3}+u_{k, 5} c^{\frac{k-4}{2}}\|T\|^{k-4}\left(\lambda_{i}^{t}\right)^{5}+\ldots+u_{k, k+1}\left(\lambda_{i}^{t}\right)^{k+1}
$$

onde, $u_{k, 0}=u_{k-1,1}, \quad u_{k, 1}=2 u_{k-1,2}, \quad u_{k, 2}=3 u_{k-1,3}+u_{k-1,1}, \ldots, \quad u_{k, j}=(j+1) u_{k-1, j+1}+$ $(j-1) u_{k-1, j-1}, \ldots, \quad u_{k, k+1}=k u_{k-1, k}$. Observe que quando $k$ é ímpar, o $j$, do $u_{k, j}$, é um número natural par e quando $k$ é par o $j$ do $u_{k, j}$ é ímpar.

Por (2.21), temos $u_{1,2}=1$. Para $k=2$, utilizando (2.22), temos

$$
\frac{\partial^{2} \lambda_{i}^{t}}{\partial t^{2}}=2 c\|T\|^{2} \lambda_{i}^{t}+2\left(\lambda_{i}^{t}\right)^{3}=2 u_{1,2} c^{\frac{2}{2}}\|T\|^{2} \lambda_{i}^{t}+2 u_{1,2}\left(\lambda_{i}^{t}\right)^{2+1}=u_{2,1} c^{\frac{2}{2}}\|T\|^{2} \lambda_{i}^{t}+u_{2,3}\left(\lambda_{i}^{t}\right)^{2+1},
$$

que verifica $(2.24)$.

Por hipótese de indução, suponha que (2.23) e (2.24) se verificam para $k-1$. Mostraremos que valem para $k$.

Se $k$ é par então $k-1$ é ímpar e, portanto, vale (2.23), ou seja,

$$
\begin{aligned}
\frac{\partial^{k-1} \lambda_{i}^{t}}{\partial t^{k-1}}= & u_{k-1,0} c^{\frac{k}{2}}\|T\|^{k}+u_{k-1,2} c^{\frac{k-2}{2}}\|T\|^{k-2}\left(\lambda_{i}^{t}\right)^{2}+u_{k-1,4} c^{\frac{k-4}{2}}\|T\|^{k-4}\left(\lambda_{i}^{t}\right)^{4}+ \\
& +\ldots+u_{k-1, k}\left(\lambda_{i}^{t}\right)^{k}
\end{aligned}
$$

Derivando a expressão (2.25), em relação a $t$, obtemos 


$$
\begin{aligned}
\frac{\partial^{k} \lambda_{i}^{t}}{\partial t^{k}}= & 2 u_{k-1,2} c^{\frac{k-2}{2}}\|T\|^{k-2}\left(\lambda_{i}^{t}\right)\left(\frac{\partial \lambda_{i}^{t}}{\partial t}\right)+4 u_{k-1,4} c^{\frac{k-4}{2}}\|T\|^{k-4}\left(\lambda_{i}^{t}\right)^{3}\left(\frac{\partial \lambda_{i}^{t}}{\partial t}\right)+\ldots+ \\
& +k u_{k-1, k}\left(\lambda_{i}^{t}\right)^{k-1}\left(\frac{\partial \lambda_{i}^{t}}{\partial t}\right) \\
= & 2 u_{k-1,2} c^{\frac{k-2}{2}}\|T\|^{k-2}\left(\lambda_{i}^{t}\right)\left(c\|T\|^{2}+\left(\lambda_{i}^{t}\right)^{2}\right)+4 u_{k-1,4} c^{\frac{k-4}{2}}\|T\|^{k-4}\left(\lambda_{i}^{t}\right)^{3}\left(c\|T\|^{2}+\left(\lambda_{i}^{t}\right)^{2}\right)+ \\
& +\ldots+k u_{k-1, k}\left(\lambda_{i}^{t}\right)^{k-1}\left(c\|T\|^{2}+\left(\lambda_{i}^{t}\right)^{2}\right) \\
= & 2 u_{k-1,2} c^{\frac{k}{2}}\|T\|^{k}\left(\lambda_{i}^{t}\right)+\left(2 u_{k-1,2}+4 u_{k-1,4}\right) c^{\frac{k-2}{2}}\|T\|^{k-2}\left(\lambda_{i}^{t}\right)^{3}+\ldots+k u_{k-1, k}\left(\lambda_{i}^{t}\right)^{k+1} \\
= & u_{k, 1} c^{\frac{k}{2}}\|T\|^{k}\left(\lambda_{i}^{t}\right)+u_{k, 3} c^{\frac{k-2}{2}}\|T\|^{k-2}\left(\lambda_{i}^{t}\right)^{3}+\ldots+u_{k, k+1}\left(\lambda_{i}^{t}\right)^{k+1} .
\end{aligned}
$$

Logo, vale (2.24). Analogamente mostra-se que vale (2.23).

Assim, como $\lambda_{n}=0$, temos que $u(0)=\sum_{i=1}^{n-1} \lambda_{i}=C_{1}$, com $C_{1}$ constante, e

$$
u^{\prime}(0)=\sum_{i=1}^{n-1} c\|T\|^{2}+\lambda_{i}^{2}
$$

o que implica em $\sum_{i=1}^{n} \lambda_{i}^{2}=C_{2}$, com $C_{2}$ constante. Segue que,

$$
u^{\prime \prime}(0)=(n-1) 2 c\|T\|^{2} \sum_{i=1}^{n-1} \lambda_{i}+2 \sum_{i=1}^{n-1} \lambda_{i}^{3}=(n-1) 2 c\|T\|^{2} C_{1}+2 \sum_{i=1}^{n-1} \lambda_{i}^{3}
$$

e daí $\sum_{i=1}^{n} \lambda_{i}^{3}=C_{3}$, com $C_{3}$ constante.

Se $k$ é par temos

$$
\begin{aligned}
u^{k}(0)= & u_{k, 1} c^{\frac{k}{2}}\|T\|^{k} \sum_{i=1}^{n-1} \lambda_{i}+u_{k, 3} c^{\frac{k-2}{2}}\|T\|^{k-2} \sum_{i=1}^{n-1}\left(\lambda_{i}\right)^{3}+u_{k, 5} c^{\frac{k-4}{2}}\|T\|^{k-4} \sum_{i=1}^{n-1}\left(\lambda_{i}\right)^{5}+ \\
& +\ldots+u_{k, k+1} \sum_{i=1}^{n-1}\left(\lambda_{i}\right)^{k+1} \\
= & u_{k, 1} c^{\frac{k}{2}}\|T\|^{k} C_{1}+u_{k, 3} c^{\frac{k-2}{2}}\|T\|^{k-2} C_{3}+u_{k, 5} c^{\frac{k-4}{2}}\|T\|^{k-4} C_{5}+\ldots+u_{k, k+1} \sum_{i=1}^{n-1}\left(\lambda_{i}\right)^{k+1} .
\end{aligned}
$$

Logo, $\sum_{i=1}^{n} \lambda_{i}^{k+1}$ é constante. Obtemos o mesmo para $k$ ímpar procedendo de maneira análoga. Portanto concluimos que $\sum_{i=1}^{n} \lambda_{i}^{k}, 1 \leq k \leq n$, é constante e pela Proposição 2.3.4 temos para 
$i \in\{1, \ldots, n\}$, que as funções $\lambda_{i}$ são constantes.

Com o Teorema 2.3.5 observamos que, a princípio, não podemos garantir que uma hipersuperfície isoparamétrica, no sentido clássico, em $\mathbb{Q}_{c}^{n} \times \mathbb{R}$ tenha suas curvaturas principais constantes. No entanto, para uma hipersuperfície de ângulo constante, ou seja, com a função $\nu$ constante, com $\nu \neq 1$, dadas no Corolário 1.1.12, obtemos o seguinte resultado.

Corolário 2.3.6. Seja $f: M^{n} \rightarrow \mathbb{Q}_{c}^{n} \times \mathbb{R}$ uma hipersuperfície de ângulo constante e $T \neq 0$. Então $f$ é isoparamétrica se, e somente se, as suas curvaturas principais são constantes.

Demonstração. Pela Observação 1.1.3, $T$ é uma direção principal. Por hipótese, $f$ possui a função $\nu$ constante e por (1.2), segue que $\|T\|$ é constante. Além disso, por (1.6), $\lambda_{n}=0 \mathrm{e}$ portanto, por (2.15), $\lambda_{n}^{t}=0$.

Suponha que as curvaturas principais $\lambda_{i}$ de $f$ são constantes. Então por (2.14) e (2.15), temos que as curvaturas principais da família de paralelas $f_{t}$ também são constantes e, portanto, $f$ é isoparamétrica.

Reciprocamente, se $f$ é isoparamétrica então, como por hipótese $\|T\|$ é constante, pelo Teorema 2.3.5, as curvaturas principais de $f$ são constantes. 


\section{Capítulo 3}

\section{Hipersuperfícies com curvaturas principais constantes em $\mathbb{Q}_{c}^{n} \times \mathbb{R}$}

Seja $M^{n}$ uma variedade Riemanniana conexa $n$-dimensional. Neste capítulo estudamos hipersuperfícies $f: M^{n} \rightarrow \mathbb{Q}_{c}^{n} \times \mathbb{R}$ com curvaturas principais constantes (CPC) cujas multiplicidades são constantes. Denotamos por $g$ o número de curvaturas principais distintas. Apresentamos alguns resultados relacionados com as multiplicidades das curvaturas principais e também resultados de classificação local dessas hipersuperfícies.

Estamos sempre considerando diferenciáveis a função $\nu$ e o campo $T$, ambos definidos em (1.1), por $\partial / \partial t=d f(T)+\nu \eta$. Consequentemente, uma vez que $\partial / \partial t$ é um campo unitário, temos $\nu^{2}+\|T\|^{2}=1$. Seja $\theta: M^{n} \rightarrow \mathbb{R}$ a função ângulo tal que $\theta(p)$ é o ângulo entre $\partial / \partial t \mathrm{e}$ $\eta(p)$. Assim,

$$
\nu=\langle\partial / \partial t, \eta\rangle=\|\partial / \partial t\|\|\eta\| \cos (\theta)=\cos (\theta) .
$$

Sabemos que a função cosseno se anula em pontos isolados, portanto, estudamos a função $\nu$ nos abertos $U \subset M$ tais que $\nu(p) \neq 0$, para todo $p \in U$, e quando $\nu \equiv 0$.

\subsection{Hipersuperfícies com curvaturas principais cons- tantes que possuem o campo $T$ como uma direção principal}

Nesta seção apresentamos um resultado de classificação das hipersuperfícies de $\mathbb{Q}_{c}^{n} \times \mathbb{R}$ com curvaturas principais constantes que possuem o campo $T$ como uma direção principal. Vale a pena lembrar que as hipersuperfícies rotacionais possuem $T$ como uma direção principal, porém a recíproca nem sempre é verdadeira. 
Utilizaremos o seguinte lema técnico.

Lema 3.1.1. Seja $a: I \subset \mathbb{R} \rightarrow \mathbb{R}$ uma função diferenciável com $a^{\prime}(s)>0$ e $a^{\prime \prime}(s) \neq 0$, para todo $s \in I$. As soluções da equação $a^{\prime \prime \prime}\left(1+\left(a^{\prime}\right)^{2}\right)-3\left(a^{\prime \prime}\right)^{2} a^{\prime}=0$ são dadas por

$$
a(s)=-\frac{\sqrt{1-\left(c_{1} s+c_{2}\right)^{2}}}{c_{1}}+c_{3}
$$

onde $c_{1}, c_{2}$ e $c_{3}$ são constantes em $\mathbb{R}$, com $c_{1} \neq 0$.

Demonstração. Seja $a^{\prime}(s)=y(s)>0$, para todo $s \in I$, então $a^{\prime \prime}(s)=y^{\prime}(s)$ e $a^{\prime \prime \prime}(s)=y^{\prime \prime}(s)$. Considerando $y$ como a variável independente, seja $u(y):=y^{\prime}(s)$. Assim,

$$
a^{\prime \prime \prime}(s)=y^{\prime \prime}(s)=\frac{\partial u(y)}{\partial s}=\frac{\partial u(y)}{\partial y} \frac{\partial y(s)}{\partial s}=u^{\prime}(y) y^{\prime}(s)=u^{\prime}(y) u(y)
$$

Sem perda da generalidade, suponha $u(y)>0$. Substituindo em $a^{\prime \prime \prime}\left(1+\left(a^{\prime}\right)^{2}\right)-3\left(a^{\prime \prime}\right)^{2} a^{\prime}=0$ obtemos

$$
u^{\prime}(y) u(y)\left(1+y^{2}\right)-3 u^{2}(y) y=0 .
$$

Observe que $a^{\prime \prime} \neq 0$ implica em $u(y) \neq 0$ e portanto, $\frac{u^{\prime}(y)}{u(y)}=\frac{3 y}{1+y^{2}}$. Então,

$$
\int \frac{u^{\prime}(y)}{u(y)} d y=\int \frac{3 y}{1+y^{2}} d y
$$

e consequentemente

$$
\ln (u(y))=\frac{3}{2} \ln \left(1+y^{2}\right)+k
$$

onde $k$ é uma constante. Logo $e^{\ln (u(y))}=e^{\ln \left(1+y^{2}\right)^{\frac{3}{2}}} e^{k}$ implica em $u(y)=\left(1+y^{2}\right)^{\frac{3}{2}} e^{k}$ e portanto $\frac{u(y)}{\left(1+y^{2}\right)^{\frac{3}{2}}}=e^{k}$. Dessa forma, $\frac{y^{\prime}(s)}{\left(1+y^{2}\right)^{\frac{3}{2}}}=e^{k}=c_{1}>0$. Integrando a última igualdade em relação a $s$, obtemos

$$
\int \frac{y^{\prime}(s)}{\left(1+y^{2}\right)^{\frac{3}{2}}} d s=\int c_{1} d s .
$$

Portanto, $0<\frac{y}{\left(1+y^{2}\right)^{\frac{1}{2}}}=c_{1} s+c_{2}$, isto é, $\frac{y^{2}}{1+y^{2}}=\left(c_{1} s+c_{2}\right)^{2}$, onde $c_{2}$ é uma constante em $\mathbb{R}$. Observe que $0<y^{2}<1+y^{2}$, ou seja, $0<\frac{y^{2}}{1+y^{2}}<1$. Assim,

$$
0<\left(c_{1} s+c_{2}\right)^{2}<1
$$


Dessa forma, $y^{2}\left(1-\left(c_{1} s+c_{2}\right)^{2}\right)=\left(c_{1} s+c_{2}\right)^{2}$, isto é, $y^{2}=\frac{\left(c_{1} s+c_{2}\right)^{2}}{1-\left(c_{1} s+c_{2}\right)^{2}}$. Logo,

$$
y=\frac{\left(c_{1} s+c_{2}\right)}{\sqrt{1-\left(c_{1} s+c_{2}\right)^{2}}} .
$$

Como $y(s)=a^{\prime}(s)$ temos $a^{\prime}(s)=\frac{\left(c_{1} s+c_{2}\right)}{\sqrt{1-\left(c_{1} s+c_{2}\right)^{2}}}$.

Integrando $a^{\prime}(s)$ em relação a s, obtemos

$$
a(s)=-\frac{\sqrt{1-\left(c_{1} s+c_{2}\right)^{2}}}{c_{1}}+c_{3},
$$

onde $c_{3}$ é uma constante em $\mathbb{R}$.

O próximo teorema classifica localmente as hipersuperfícies de $\mathbb{Q}_{c}^{n} \times \mathbb{R}$ com curvaturas principais constantes que possuem o campo $T$ como uma direção principal e $\nu(p) \neq 0$, para todo $p \in M$.

Teorema 3.1.2. Seja $f: M^{n} \rightarrow \mathbb{Q}_{c}^{n} \times \mathbb{R}, n \geq 2$, uma hipersuperfície com curvaturas principais constantes que possui o campo $T$ como uma direção principal e $\nu(p) \neq 0$, para todo $p \in M$. Então $c=-1$ e $f$ é localmente dada por $f(x, s)=h_{s}(x)+B s \partial / \partial t$, para algum $B \in \mathbb{R}, B>0$, onde $h_{s}$ é uma família de horosferas em $\mathbb{H}^{n}$. Além disso, a curvatura principal correspondente ao campo $T$ é $\lambda=0$ e as outras curvaturas principais são todas iguais a $\frac{B}{\sqrt{1+B^{2}}}$ ou $-\frac{B}{\sqrt{1+B^{2}}}$.

Demonstração. Vimos, no Teorema 1.1.11, que se $T$ é uma direção principal de $f$ e $\nu(p) \neq 0$, para todo $p \in M$, então $f$ é localmente dada por $f: M^{n}=M^{n-1} \times I \rightarrow \mathbb{Q}_{c}^{n} \times \mathbb{R}$, com $f(x, s)=h_{s}(x)+a(s) \partial / \partial t$, onde $a: I \rightarrow \mathbb{R}$ é uma função diferenciável satisfazendo $a^{\prime}(s)>0$ para todo $s \in I$. Além disso, pela Proposição 1.1.14, $A_{\eta} X=-\frac{a^{\prime}(s)}{b(s)} A^{s} X$, para todo $X \in T M^{n-1}$, e em particular, $A_{\eta} X_{i}=-\frac{a^{\prime}(s)}{b(s)} \lambda_{i}^{s}(x) X_{i}$, para as direções principais $X_{i} \in T M^{n-1}$ de $h$ e $A_{\eta} T=\frac{a^{\prime \prime}(s)}{b^{3}(s)} T$, onde $b(s)=\sqrt{1+\left(a^{\prime}(s)\right)^{2}}$. Portanto

$$
A_{\eta}\left(X_{i}\right)=\mu_{i}(x, s) X_{i}, \quad \text { com } \quad \mu_{i}(x, s)=-\frac{a^{\prime}(s)}{b(s)} \lambda_{i}^{s}(x)
$$

para $i \in\{1, \ldots, n-1\}$ e $A_{\eta} T=\mu_{n}(x, s) T, \operatorname{com} \mu_{n}(x, s)=\frac{a^{\prime \prime}(s)}{b^{3}(s)}$. 
Pela Proposição 1.1.15, temos

$$
\lambda_{i}^{s}(x)=\frac{c S_{c}(s)+C_{c}(s) \lambda_{i}(x)}{C_{c}(s)-S_{c}(s) \lambda_{i}(x)}
$$

e portanto

$$
\mu_{i}(x, s)=\left(-\frac{a^{\prime}(s)}{b(s)}\right)\left(\frac{c S_{c}(s)+C_{c}(s) \lambda_{i}(x)}{C_{c}(s)-S_{c}(s) \lambda_{i}(x)}\right), \quad i \in\{1, \ldots, n-1\}
$$

Analisaremos sob quais condições as funções $\mu_{i}$ são constantes.

Observe que

$$
\begin{aligned}
\frac{\partial \lambda_{i}^{s}}{\partial x} & =\frac{C_{c}(s) \lambda_{i}^{\prime}(x)\left(C_{c}(s)-S_{c}(s) \lambda_{i}(x)\right)-\left(c S_{c}(s)+C_{c}(s) \lambda_{i}(x)\right)\left(-S_{c}(s) \lambda_{i}^{\prime}(x)\right)}{\left(C_{c}(s)-S_{c}(s) \lambda_{i}(x)\right)^{2}} \\
& =\frac{\lambda_{i}^{\prime}(x)}{\left(C_{c}(s)-S_{c}(s) \lambda_{i}(x)\right)^{2}},
\end{aligned}
$$

e

$$
\begin{aligned}
\frac{\partial \lambda_{i}^{s}}{\partial s}= & \frac{\left(c C_{c}(s)-c S_{c}(s) \lambda_{i}(x)\right)\left(C_{c}(s)-S_{c}(s) \lambda_{i}(x)\right)}{\left(C_{c}(s)-S_{c}(s) \lambda_{i}(x)\right)^{2}}+ \\
& -\frac{\left(c S_{c}(s)+C_{c}(s) \lambda_{i}(x)\right)\left(-c S_{c}(s)-C_{c}(s) \lambda_{i}(x)\right)}{\left(C_{c}(s)-S_{c}(s) \lambda_{i}(x)\right)^{2}} \\
= & \frac{c\left(C_{c}(s)-S_{c}(s) \lambda_{i}(x)\right)^{2}+\left(c S_{c}(s)+C_{c}(s) \lambda_{i}(x)\right)^{2}}{\left(C_{c}(s)-S_{c}(s) \lambda_{i}(x)\right)^{2}} \\
= & c+\left(\lambda_{i}^{s}(x)\right)^{2} .
\end{aligned}
$$

Ou seja,

$$
\frac{\partial \lambda_{i}^{s}}{\partial x}=\frac{\lambda_{i}^{\prime}(x)}{\left(C_{c}(s)-S_{c}(s) \lambda_{i}(x)\right)^{2}}
$$

e

$$
\frac{\partial \lambda_{i}^{s}}{\partial s}=c+\left(\lambda_{i}^{s}(x)\right)^{2}
$$

Para $i \in\{1, \ldots, n-1\}, \frac{\partial \mu_{i}}{\partial x}=-\frac{a^{\prime}(s)}{b(s)} \frac{\partial \lambda_{i}^{s}}{\partial x}=0$ se e somente se, $\frac{\partial \lambda_{i}^{s}}{\partial x}=0$.

Portanto para que $\mu_{i}$ seja constante em relação a $x$ é necessário, por (3.2), que $h$ seja isoparamétrica, ou seja, que as funções $\lambda_{i}$ sejam constantes para todo $i \in\{1, \ldots, n-1\}$.

Além disso,

$$
\frac{\partial \mu_{i}}{\partial s}=\frac{\left(-a^{\prime \prime} b+a^{\prime} b^{\prime}\right)}{b^{2}} \lambda_{i}^{s}-\frac{a^{\prime}}{b} \frac{\partial \lambda_{i}^{s}}{\partial s}=-\frac{a^{\prime \prime}}{b^{3}} \lambda_{i}^{s}-\frac{a^{\prime}}{b}\left(c+\left(\lambda_{i}^{s}\right)^{2}\right)=\frac{-a^{\prime \prime} \lambda_{i}^{s}-a^{\prime} b^{2}\left(c+\left(\lambda_{i}^{s}\right)^{2}\right)}{b^{3}} .
$$

Assim, para $i \in\{1, \ldots, n-1\}, \frac{\partial \mu_{i}}{\partial s}=0$ se, e somente se, $a^{\prime \prime} \lambda_{i}^{s}+a^{\prime}\left(1+a^{\prime 2}\right)\left(c+\left(\lambda_{i}^{s}\right)^{2}\right)=0$. 
Agora vamos analisar a curvatura $\mu_{n}(x, s)=\frac{a^{\prime \prime}(s)}{b^{3}(s)}$. Temos $\frac{\partial \mu_{n}}{\partial x}=0 \mathrm{e}$

$$
\frac{\partial \mu_{n}}{\partial s}=\frac{a^{\prime \prime \prime} b^{3}-3 a^{\prime \prime} b^{2} b^{\prime}}{b^{6}}=\frac{a^{\prime \prime \prime} b-3 a^{\prime \prime} b^{\prime}}{b^{4}}
$$

Consequentemente, $\frac{\partial \mu_{n}}{\partial s}=0$ se, e somente se, $a^{\prime \prime \prime} b-3 a^{\prime \prime} b^{\prime}=0$, ou seja, $a^{\prime \prime \prime}\left(1+a^{\prime 2}\right)-3 a^{\prime \prime 2} a^{\prime}=0$.

Analisaremos, para todo $s \in I$ e $i \in\{1, \ldots, n-1\}$, as seguintes equações,

$$
\begin{gathered}
a^{\prime \prime} \lambda_{i}^{s}+a^{\prime}\left(1+a^{\prime 2}\right)\left(c+\left(\lambda_{i}^{s}\right)^{2}\right)=0 \quad \mathrm{e} \\
a^{\prime \prime \prime}\left(1+a^{\prime 2}\right)-3 a^{\prime \prime 2} a^{\prime}=0 .
\end{gathered}
$$

Como a função $a$ é diferenciável, de classe $\mathcal{C}^{\infty}$, podemos considerar apenas dois casos, $a^{\prime \prime}(s)=$ 0 , para todo $s \in I$, ou $a^{\prime \prime}(s) \neq 0$, para todo $s \in I$, se necessário restringindo o intervalo $I \in \mathbb{R}$.

Caso 1: Suponha $a^{\prime \prime}(s)=0$ para todo $s \in I$. Então $a^{\prime \prime \prime}(s)=0$ e consequentemente a equação (3.5) é satisfeita. Nesse caso, pela equação (3.4), temos que $a^{\prime}\left(1+a^{\prime 2}\right)\left(c+\left(\lambda_{i}^{s}\right)^{2}\right)=0$. Como $a^{\prime}(s)>0$, concluimos que $c+\left(\lambda_{i}^{s}\right)^{2}=0$.

Se $c=1$ então $1+\left(\lambda_{i}^{s}\right)^{2} \neq 0$. Portanto não podemos ter esse caso para $c=1$.

Se $c=-1$ então $\left(\lambda_{i}^{s}\right)^{2}=1$. Assim, $\lambda_{i}^{s}= \pm 1$, o que implica em $\lambda_{i}= \pm 1$. Além disso, $\mu_{n}=0$ e $\mu_{i}= \pm \frac{a^{\prime}}{b}$, para $i \in\{1, \ldots, n-1\}$.

Caso 2: Suponha $a^{\prime \prime}(s) \neq 0$, para todo $s \in I$. Portanto, pelo Lema 3.1.1, temos que as soluções da equação (3.5) são dadas por $a(s)=-\frac{\sqrt{1-\left(c_{1} s+c_{2}\right)^{2}}}{c_{1}}+c_{3}$, onde $c_{1}$, $c_{2}$ e $c_{3}$ são constantes em $\mathbb{R}$ com $c_{1} \neq 0$. Vamos verificar se essas soluções satisfazem a equação (3.4). Observe que

$$
\begin{aligned}
& a^{\prime}(s)=-\frac{1}{2 c_{1}} \frac{\left(-2\left(c_{1} s+c_{2}\right) c_{1}\right)}{\sqrt{1-\left(c_{1} s+c_{2}\right)^{2}}}=\frac{c_{1} s+c_{2}}{\sqrt{1-\left(c_{1} s+c_{2}\right)^{2}}} \quad \mathrm{e} \\
a^{\prime \prime}(s)= & \left(c_{1} \sqrt{1-\left(c_{1} s+c_{2}\right)^{2}}-\left(c_{1} s+c_{2}\right) \frac{\left(-2 c_{1}\left(c_{1} s+c_{2}\right)\right)}{2 \sqrt{1-\left(c_{1} s+c_{2}\right)^{2}}}\right) \frac{1}{1-\left(c_{1} s+c_{2}\right)^{2}} \\
= & \frac{c_{1}-c_{1}\left(c_{1} s+c_{2}\right)^{2}+c_{1}\left(c_{1} s+c_{2}\right)^{2}}{\left(1-\left(c_{1} s+c_{2}\right)^{2}\right)^{\frac{3}{2}}} \\
= & \frac{c_{1}}{\left(1-\left(c_{1} s+c_{2}\right)^{2}\right)^{\frac{3}{2}}} .
\end{aligned}
$$


Então

$$
\begin{aligned}
a^{\prime}\left(1+a^{\prime 2}\right) & =\frac{c_{1} s+c_{2}}{\sqrt{1-\left(c_{1} s+c_{2}\right)^{2}}}\left(1+\frac{\left(c_{1} s+c_{2}\right)^{2}}{1-\left(c_{1} s+c_{2}\right)^{2}}\right) \\
& =\frac{\left(c_{1} s+c_{2}\right)\left(1-\left(c_{1} s+c_{2}\right)^{2}+\left(c_{1} s+c_{2}\right)^{2}\right)}{\left(1-\left(c_{1} s+c_{2}\right)^{2}\right)^{\frac{3}{2}}} \\
& =\frac{c_{1} s+c_{2}}{\left(1-\left(c_{1} s+c_{2}\right)^{2}\right)^{\frac{3}{2}}} .
\end{aligned}
$$

Assim,

$$
a^{\prime \prime} \lambda_{i}^{s}+a^{\prime}\left(1+a^{\prime 2}\right)\left(c+\left(\lambda_{i}^{s}\right)^{2}\right)=\frac{c_{1}}{\left(1-\left(c_{1} s+c_{2}\right)^{2}\right)^{\frac{3}{2}}} \lambda_{i}^{s}+\frac{c_{1} s+c_{2}}{\left(1-\left(c_{1} s+c_{2}\right)^{2}\right)^{\frac{3}{2}}}\left(c+\left(\lambda_{i}^{s}\right)^{2}\right)
$$

Portanto as soluções $a(s)$ da equação (3.5) satisfazem a equação (3.4) se, e somente se,

$$
c_{1} \lambda_{i}^{s}+\left(c_{1} s+c_{2}\right)\left(c+\left(\lambda_{i}^{s}\right)^{2}\right)=0
$$

para todo $s \in I$.

Suponha que vale a fórmula (3.6), para todo $s \in I$. Se $c+\left(\lambda_{i}^{s}\right)^{2}=0$, então teremos $c_{1} \lambda_{i}^{s}=0$, isto é, $\lambda_{i}^{s}=0$, para todo $s \in I$, já que $c_{1} \neq 0$. Mas,

$$
\lambda_{i}^{s}(x)=\frac{c S_{c}(s)+C_{c}(s) \lambda_{i}(x)}{C_{c}(s)-S_{c}(s) \lambda_{i}(x)}=0 \text { implica em } c S_{c}(s)+C_{c}(s) \lambda_{i}(x)=0,
$$

para todo $s \in I$, o que não pode ocorrer. Logo, nesse caso, não podemos ter $c+\left(\lambda_{i}^{s}\right)^{2}=0$.

Além disso, derivando a equação (3.6) temos

$$
\frac{\partial}{\partial s}\left(c_{1} \lambda_{i}^{s}+\left(c_{1} s+c_{2}\right)\left(c+\left(\lambda_{i}^{s}\right)^{2}\right)\right)=c_{1} \frac{\partial \lambda_{i}^{s}}{\partial s}+c_{1}\left(c+\left(\lambda_{i}^{s}\right)^{2}\right)+2\left(c_{1} s+c_{2}\right) \lambda_{i}^{s} \frac{\partial \lambda_{i}^{s}}{\partial s}=0
$$

Dessa forma, pela equação (3.3), segue que

$$
2 c_{1}\left(c+\left(\lambda_{i}^{s}\right)^{2}\right)+2\left(c_{1} s+c_{2}\right) \lambda_{i}^{s}\left(c+\left(\lambda_{i}^{s}\right)^{2}\right)=2\left(c+\left(\lambda_{i}^{s}\right)^{2}\right)\left(c_{1}+\left(c_{1} s+c_{2}\right) \lambda_{i}^{s}\right)=0 .
$$

Consequentemente, $c_{1}+\left(c_{1} s+c_{2}\right) \lambda_{i}^{s}=0$. Vimos, em (3.1), que $c_{1} s+c_{2}>0$, para todo $s \in I$ e assim, $\lambda_{i}^{s}=\frac{-c_{1}}{c_{1} s+c_{2}}$. Logo, por um lado,

$$
\frac{\partial \lambda_{i}^{s}}{\partial s}=\frac{\left(c_{1}\right)^{2}}{\left(c_{1} s+c_{2}\right)^{2}}=\left(\lambda_{i}^{s}\right)^{2}
$$

Mas, por outro lado, temos, por (3.3), que $\frac{\partial \lambda_{i}^{s}}{\partial s}=c+\left(\lambda_{i}^{s}\right)^{2}$, o que implica em $c=0$, o que não pode ocorrer pois estamos considerando $c=1$ ou $c=-1$. Logo não vale a equação (3.6) 
e portanto as soluções da equação (3.5) não são soluções da equação (3.4) com a condição $a^{\prime \prime}(s) \neq 0$, para todo $s \in I$.

Assim, concluimos que $a^{\prime \prime}=0$ e, nesse caso, $a(s)=B s$, com $B \in \mathbb{R}, B>0$ pois $a^{\prime}(s)>0$ e a função $\nu$ é constante, pelo Corolário 1.1.12. Portanto, $\lambda=\mu_{n}=0$ e $\mu_{i}=\frac{B}{\sqrt{1+B^{2}}}$ ou $\mu_{i}=-\frac{B}{\sqrt{1+B^{2}}}$, para $i \in\{1, \ldots, n-1\}$.

Observação 3.1.3. Pela Proposição 1.1.10, para $n \geq 3$, e pela Observação 7 (i) de [32], para $n=2$, as hipersuperfícies dadas pelo Teorema 3.1.2, são rotacionais em $\mathbb{H}^{n} \times \mathbb{R}$ cujas órbitas são horosferas.

\subsection{Multiplicidade das curvaturas principais}

A seguir, apresentamos alguns resultados relacionados com as multiplicidades das curvaturas principais de hipersuperfícies em $\mathbb{Q}_{c}^{n} \times \mathbb{R}$.

Observe que, pela Proposição 1.2.1, as curvaturas principais de uma hipersuperfície CPC em $\mathbb{Q}_{c}^{n} \times \mathbb{R}$ com $g=2$, possuem multiplicidades constantes.

Teorema 3.2.1. Seja $f: M^{n} \rightarrow \mathbb{Q}_{c}^{n} \times \mathbb{R}, n \geq 2$, uma hipersuperficie com curvaturas principais constantes e de multiplicidades constantes, não umbilica e com a função $\nu \neq 0$. Então existe pelo menos uma curvatura principal com multiplicidade igual a 1.

Demonstração. Seja $\left\{X_{1}, X_{2}, \ldots, X_{n}\right\}$ um referencial local ortonormal de direções principais de $f$. Podemos escrever $T=\sum_{i=1}^{n} b_{i} X_{i}$. Seja $g$ o número de curvaturas principais distintas. Como $f$ é não umbílica temos que $g \geq 2$.

Se $n=2$ então existem duas curvaturas principais distintas e portanto cada uma possui multiplicidade 1.

Se $n=3$ então $g=2$ ou $g=3$. Se $g=2$, uma curvatura tem multiplicidade 2 e a outra tem multiplicidade 1 . Se $g=3$ cada curvatura principal tem multiplicidade 1 .

Se $n \geq 4$, suponha que todas as curvaturas principais tenham multiplicidade maior ou igual a 2 . Nesse caso, $2 \leq g \leq \frac{n}{2}$, se $n$ for par e $2 \leq g \leq \frac{n-1}{2}$, se $n$ for ímpar. Dado $\rho \in\{1, \ldots, g\}$, seja $B_{\rho}=\left\{i \in\{1, \ldots, n\} / A X_{i}=\lambda_{\rho} X_{i}\right\}$. Observe que $B_{\rho}$ possui pelo menos 2 elementos.

Dado $\rho$, considere a equação de Codazzi,

$$
\nabla_{X_{i}} A X_{j}-\nabla_{X_{j}} A X_{i}-A\left[X_{i}, X_{j}\right]=c \nu\left(b_{j} X_{i}-b_{i} X_{j}\right)
$$


para $i, j \in B_{\rho}$. Temos que $\nabla_{X_{i}} A X_{j}-\nabla_{X_{j}} A X_{i}=\lambda_{\rho}\left[X_{i}, X_{j}\right]=\lambda_{\rho} \sum_{k=1}^{n}\left\langle\left[X_{i}, X_{j}\right], X_{k}\right\rangle X_{k}$ e $A\left[X_{i}, X_{j}\right]=\sum_{k=1}^{n}\left\langle\left[X_{i}, X_{j}\right], X_{k}\right\rangle A X_{k}$. Assim,

$$
\nabla_{X_{i}} A X_{j}-\nabla_{X_{j}} A X_{i}-A\left[X_{i}, X_{j}\right]=\sum_{k \notin B_{\rho}}\left\langle\left[X_{i}, X_{j}\right], X_{k}\right\rangle\left(\lambda_{\rho} X_{k}-A X_{k}\right)
$$

Então, por (3.7) e (3.8), temos

$$
\sum_{k \notin B_{\rho}}\left\langle\left[X_{i}, X_{j}\right], X_{k}\right\rangle\left(\lambda_{\rho}-\lambda_{k}\right) X_{k}-c \nu b_{j} X_{i}+c \nu b_{i} X_{j}=0
$$

Como $i, j \in B_{\rho}$ com $i \neq j, k \notin B_{\rho}$ e $\nu \neq 0$ temos que ter $b_{i}=b_{j}=0$ para todo $i, j \in B_{\rho}$, ou seja, $T$ não possui componentes nas direções cujas curvaturas principais correspondentes possuem multiplicidade maior ou igual a 2. Assim, supondo que não existem curvaturas principais de multiplicidade 1 , concluimos que $T=0$. Isso implica que $f\left(M^{n}\right)$ é um subconjunto aberto de uma fatia $\mathbb{Q}_{c}^{n} \times\{t\}$ e portanto é totalmente geodésica, o que contradiz a hipótese que $g \geq 2$. Logo, $f$ possui pelo menos uma curvatura principal com multiplicidade igual a 1.

Observação 3.2.2. Pela demonstração do teorema anterior temos que $T$ não tem componentes nas direções cujas curvaturas principais correspondentes possuem multiplicidade maior ou igual a 2.

Observação 3.2.3. O Teorema 3.2.1 vale também para $\nu \equiv 0$ se $c=-1$, pois a curvatura correspondente ao fator $\mathbb{R}$ é $\lambda=0$ e as demais são não nulas, pelo Teorema 1.2.3. É válido para $\nu \equiv 0$ e $c=1$ se $g=2$ e para $g=3$, excluindo os casos onde $f\left(M^{n}\right)$ é um aberto de $M^{n-1} \times \mathbb{R}$, onde $M^{n-1}$ é uma hipersuperfície de Cartan para $n \in\{7,13,25\}$.

Proposição 3.2.4. Seja $f: M^{n} \rightarrow \mathbb{Q}_{c}^{n} \times \mathbb{R}, n \geq 3$, com a função $\nu \neq 0$, uma hipersuperfície com curvaturas principais constantes e respectivas multiplicidades constantes. Se uma única curvatura principal tem multiplicidade 1, então o campo vetorial T é uma direção principal correspondente a essa curvatura. Além disso, as curvaturas com multiplicidade maior que 1 são todas não nulas.

Demonstração. Seja $\left\{X_{1}, X_{2}, \ldots, X_{n}\right\}$ um referencial local ortonormal de direções principais de $f$. Suponha, sem perda de generalidade, que $X_{n}$ é associado a $\lambda$, isto é, $A X_{n}=\lambda X_{n}$. Pela Observação 3.2.2, se $\lambda$ é a única curvatura com multiplicidade 1 então $T=b X_{n}$, onde $b: U \subset M^{n} \rightarrow \mathbb{R}$ é uma função diferenciável definida num aberto $U \subset M^{n}$ onde os campos $X_{1}, X_{2}, \ldots, X_{n}$ estão definidos. 
Seja $g$ o número de curvaturas principais distintas. Por hipótese, $g \geq 2$. Dado $\rho \in$ $\{1, \ldots, g-1\}$, seja $B_{\rho}=\left\{i \in\{1, \ldots, n\} / A X_{i}=\mu_{\rho} X_{i}\right\}$ com $\mu_{\rho} \neq \lambda=\mu_{g}$. Observe que $B_{\rho}$ possui pelo menos 2 elementos. Considere a equação de Codazzi

$$
\nabla_{X_{n}} A X_{i}-\nabla_{X_{i}} A X_{n}-A\left[X_{n}, X_{i}\right]=c \nu\left(b_{i} X_{n}-b_{n} X_{i}\right)
$$

para $i \in B_{\rho}$. Temos que

$$
\begin{gathered}
\nabla_{X_{n}} A X_{i}-\nabla_{X_{i}} A X_{n}=\mu_{\rho} \nabla_{X_{n}} X_{i}-\lambda \nabla_{X_{i}} X_{n} \\
A\left[X_{n}, X_{i}\right]=\sum_{k=1}^{n}\left\langle\nabla_{X_{n}} X_{i}-\nabla_{X_{i}} X_{n}, X_{k}\right\rangle A X_{k} .
\end{gathered}
$$

Portanto por (3.10) e (3.11) obtemos

$$
\nabla_{X_{n}} A X_{i}-\nabla_{X_{i}} A X_{n}-A\left[X_{n}, X_{i}\right]=\sum_{k=1}^{n}\left\langle\nabla_{X_{n}} X_{i}, X_{k}\right\rangle\left(\mu_{\rho} X_{k}-A X_{k}\right)-\sum_{k=1}^{n}\left\langle\nabla_{X_{i}} X_{n}, X_{k}\right\rangle\left(\lambda X_{k}-A X_{k}\right)
$$

Então, por (3.9),

$$
\sum_{k \notin B_{\rho}}\left\langle\nabla_{X_{n}} X_{i}, X_{k}\right\rangle\left(\mu_{\rho}-\mu_{k}\right) X_{k}-\sum_{k \neq n}\left\langle\nabla_{X_{i}} X_{n}, X_{k}\right\rangle\left(\lambda-\mu_{k}\right) X_{k}-c \nu b_{i} X_{n}+c \nu b_{n} X_{i}=0
$$

Logo,

$$
c \nu b-\left\langle\nabla_{X_{i}} X_{n}, X_{i}\right\rangle\left(\lambda-\mu_{\rho}\right)=0, \quad \forall i \in B_{\rho} .
$$

Então $b=0 \Leftrightarrow\left\langle\nabla_{X_{i}} X_{n}, X_{i}\right\rangle=0$, para $i \in B_{\rho}$.

Sabemos que $\nabla_{X} T=\nu A X$ para todo $X \in T M^{n}$. Dessa forma, temos que

$$
\nu \mu_{\rho} X_{i}=\nabla_{X_{i}} T=\nabla_{X_{i}} b X_{n}=X_{i}(b) X_{n}+b \nabla_{X_{i}} X_{n}, \quad i \in \beta_{\rho},
$$

o que implica em $\nu \mu_{\rho}\left\langle X_{i}, X_{i}\right\rangle=X_{i}(b)\left\langle X_{n}, X_{i}\right\rangle+b\left\langle\nabla_{X_{i}} X_{n}, X_{i}\right\rangle$, ou seja, $b\left\langle\nabla_{X_{i}} X_{n}, X_{i}\right\rangle=\nu \mu_{\rho}$. Portanto $c \nu b\left\langle\nabla_{X_{i}} X_{n}, X_{i}\right\rangle=c \nu^{2} \mu_{\rho}$ e pela equação (3.12), $\left\langle\nabla_{X_{i}} X_{n}, X_{i}\right\rangle^{2}\left(\lambda-\mu_{\rho}\right)=c \nu^{2} \mu_{\rho}$. Consequentemente,

$$
b=0 \Leftrightarrow\left\langle\nabla_{X_{i}} X_{n}, X_{i}\right\rangle=0, \quad \forall i \in B_{\rho} \Leftrightarrow \mu_{\rho}=0
$$

Se $\mu_{\rho}=0$ então $b=0$ e $T=0$, ou seja, $f(M)$ é totalmente geodésica, o que contradiz o fato que $g \geq 2$. Assim, $\mu_{\rho} \neq 0$ e $b \neq 0$. Logo, $T$ é uma direção principal e as curvaturas com multiplicidade maior que 1 são todas não nulas. 
Pelo Teorema 3.1.2 temos a recíproca da Proposição 3.2.4 e portanto o resultado seguinte é válido.

Proposição 3.2.5. Seja $f: M^{n} \rightarrow \mathbb{Q}_{c}^{n} \times \mathbb{R}, n \geq 3$, com a função $\nu \neq 0$, uma hipersuperfície com curvaturas principais constantes e de multiplicidades constantes. Então o campo vetorial $T$ é uma direção principal se, e somente se, existe uma única curvatura principal de multiplicidade 1. Além disso, as curvaturas com multiplicidade maior que 1 são todas não nulas.

\subsection{Hipersuperfícies de $\mathbb{Q}_{c}^{n} \times \mathbb{R}$ com curvaturas princi- pais constantes para $g \in\{1,2,3\}$}

Nesta seção apresentamos um resultado que classifica localmente as hipersuperfícies com curvaturas principais constantes. Para isso, precisamos de algumas proposições.

Proposição 3.3.1. Sejam $f: M^{2} \rightarrow \mathbb{Q}_{c}^{2} \times \mathbb{R}$ uma superfície com duas curvaturas principais constantes e distintas, $\lambda_{1}$ e $\lambda_{2}$. Seja $\left\{X_{1}, X_{2}\right\}$ um referencial ortonormal de direções principais associadas a $\lambda_{1}$ e $\lambda_{2}$. Considere $T=b_{1} X_{1}+b_{2} X_{2}$, onde $b_{1}, b_{2}: M^{n} \rightarrow \mathbb{R}$ são funções diferenciáveis. Então

$$
\lambda_{1} \lambda_{2}+2 c \nu^{2}+\frac{c\left(\lambda_{1} b_{1}^{2}-\lambda_{2} b_{2}^{2}\right)}{\lambda_{2}-\lambda_{1}}+\frac{2 \nu^{2}\left(b_{1}^{2}+b_{2}^{2}\right)}{\left(\lambda_{2}-\lambda_{1}\right)^{2}}=0
$$

Demonstração. Pela equação de Codazzi temos

$$
\nabla_{X_{1}} A X_{2}-\nabla_{X_{2}} A X_{1}-A\left[X_{1}, X_{2}\right]=c \nu\left(b_{2} X_{1}-b_{1} X_{2}\right)
$$

Observe que

$$
\nabla_{X_{1}} A X_{2}=\lambda_{2} \nabla_{X_{1}} X_{2}=\lambda_{2}\left\langle\nabla_{X_{1}} X_{2}, X_{1}\right\rangle X_{1}
$$

pois $X_{1}\left\langle X_{2}, X_{2}\right\rangle=0$. Analogamente temos,

$$
\nabla_{X_{2}} A X_{1}=\lambda_{1}\left\langle\nabla_{X_{2}} X_{1}, X_{2}\right\rangle X_{2}
$$

Assim,

$$
A\left[X_{1}, X_{2}\right]=A\left(\nabla_{X_{1}} X_{2}-\nabla_{X_{2}} X_{1}\right)=\lambda_{1}\left\langle\nabla_{X_{1}} X_{2}, X_{1}\right\rangle X_{1}-\lambda_{2}\left\langle\nabla_{X_{2}} X_{1}, X_{2}\right\rangle X_{2}
$$


Portanto, pela equação (3.14),

$$
\left(\lambda_{2}-\lambda_{1}\right)\left\langle\nabla_{X_{1}} X_{2}, X_{1}\right\rangle X_{1}+\left(\lambda_{2}-\lambda_{1}\right)\left\langle\nabla_{X_{2}} X_{1}, X_{2}\right\rangle X_{2}=c \nu\left(b_{2} X_{1}-b_{1} X_{2}\right),
$$

o que implica, uma vez que $X_{1}$ e $X_{2}$ são campos linearmente independentes, em

$$
\left(\lambda_{2}-\lambda_{1}\right)\left\langle\nabla_{X_{1}} X_{2}, X_{1}\right\rangle=c \nu b_{2} \quad \text { e } \quad\left(\lambda_{2}-\lambda_{1}\right)\left\langle\nabla_{X_{2}} X_{1}, X_{2}\right\rangle=-c \nu b_{1}
$$

ou seja,

$$
\begin{aligned}
\nabla_{X_{1}} X_{2} & =\frac{c \nu b_{2}}{\lambda_{2}-\lambda_{1}} X_{1} \\
\nabla_{X_{2}} X_{1} & =\frac{-c \nu b_{1}}{\lambda_{2}-\lambda_{1}} X_{2}
\end{aligned}
$$

E assim,

$$
\begin{aligned}
\nabla_{X_{1}} X_{1} & =\frac{-c \nu b_{2}}{\lambda_{2}-\lambda_{1}} X_{2}, \\
\nabla_{X_{2}} X_{2} & =\frac{c \nu b_{1}}{\lambda_{2}-\lambda_{1}} X_{1} .
\end{aligned}
$$

Utilizando que $\nabla_{X} T=\nu A X$, para todo $X \in T M^{2}$, obtemos

$$
\begin{gathered}
\nabla_{X_{1}} T=\nabla_{X_{1}}\left(b_{1} X_{1}+b_{2} X_{2}\right)=X_{1}\left(b_{1}\right) X_{1}+b_{1} \nabla_{X_{1}} X_{1}+X_{1}\left(b_{2}\right) X_{2}+b_{2} \nabla_{X_{1}} X_{2}=\nu \lambda_{1} X_{1} \quad \mathrm{e} \\
\nabla_{X_{2}} T=\nabla_{X_{2}}\left(b_{1} X_{1}+b_{2} X_{2}\right)=X_{2}\left(b_{1}\right) X_{1}+b_{1} \nabla_{X_{2}} X_{1}+X_{2}\left(b_{2}\right) X_{2}+b_{2} \nabla_{X_{2}} X_{2}=\nu \lambda_{2} X_{2} .
\end{gathered}
$$

Fazendo o produto interno de ambas as igualdades anteriores por $X_{1}$ e $X_{2}$, concluimos

$$
\begin{aligned}
& X_{1}\left(b_{2}\right)=b_{1}\left\langle\nabla_{X_{1}} X_{2}, X_{1}\right\rangle=\frac{c \nu b_{1} b_{2}}{\lambda_{2}-\lambda_{1}}, \\
& X_{2}\left(b_{1}\right)=b_{2}\left\langle\nabla_{X_{2}} X_{1}, X_{2}\right\rangle=\frac{-c \nu b_{1} b_{2}}{\lambda_{2}-\lambda_{1}} .
\end{aligned}
$$

Portanto $X_{1}\left(b_{2}\right)=-X_{2}\left(b_{1}\right)$. Além disso,

$$
X_{1}\left(b_{1}\right)+b_{2}\left\langle\nabla_{X_{1}} X_{2}, X_{1}\right\rangle=\nu \lambda_{1} \quad \text { e } \quad X_{2}\left(b_{2}\right)+b_{1}\left\langle\nabla_{X_{2}} X_{1}, X_{2}\right\rangle=\nu \lambda_{2} .
$$

Ou seja, 


$$
\begin{aligned}
& X_{1}\left(b_{1}\right)=\nu \lambda_{1}-\frac{c \nu\left(b_{2}\right)^{2}}{\lambda_{2}-\lambda_{1}}, \\
& X_{2}\left(b_{2}\right)=\nu \lambda_{2}+\frac{c \nu\left(b_{1}\right)^{2}}{\lambda_{2}-\lambda_{1}} .
\end{aligned}
$$

Também temos que $X(\nu)=-\langle A X, T\rangle$, para todo $X \in T M^{n}$, e portanto

$$
\begin{aligned}
& X_{1}(\nu)=-\lambda_{1} b_{1}, \\
& X_{2}(\nu)=-\lambda_{2} b_{2} .
\end{aligned}
$$

Além disso, pela equação de Gauss temos

$$
\left\langle R\left(X_{1}, X_{2}\right) X_{2}, X_{1}\right\rangle=\lambda_{1} \lambda_{2}+c \nu^{2}
$$

Observe que

$$
\begin{aligned}
\nabla_{\left[X_{1}, X_{2}\right]} X_{2} & =\nabla_{\left(\nabla_{X_{1}} X_{2}-\nabla_{X_{2}} X_{1}\right)} X_{2} \\
& =\nabla_{\left(\left\langle\nabla_{X_{1}} X_{2}, X_{1}\right\rangle X_{1}-\left\langle\nabla_{X_{2}} X_{1}, X_{2}\right\rangle X_{2}\right)} X_{2} \\
& =\left\langle\nabla_{X_{1}} X_{2}, X_{1}\right\rangle \nabla_{X_{1}} X_{2}-\left\langle\nabla_{X_{2}} X_{1}, X_{2}\right\rangle \nabla_{X_{2}} X_{2} .
\end{aligned}
$$

Assim,

$$
\left\langle\nabla_{\left[X_{1}, X_{2}\right]} X_{2}, X_{1}\right\rangle=\left\langle\nabla_{X_{1}} X_{2}, X_{1}\right\rangle^{2}+\left\langle\nabla_{X_{2}} X_{1}, X_{2}\right\rangle^{2}
$$

Então, pelas equações (3.20) e (3.15), obtemos

$$
\left\langle\nabla_{X_{1}} \nabla_{X_{2}} X_{2}-\nabla_{X_{2}} \nabla_{X_{1}} X_{2}, X_{1}\right\rangle=\lambda_{1} \lambda_{2}+c \nu^{2}+\frac{\nu^{2}\left(b_{1}^{2}+b_{2}^{2}\right)}{\left(\lambda_{2}-\lambda_{1}\right)^{2}} .
$$

Observe, pelas equações (3.15), que $\left\langle\nabla_{X_{1}} X_{2}, \nabla_{X_{2}} X_{1}\right\rangle=0$ e portanto $X_{2}\left\langle\nabla_{X_{1}} X_{2}, X_{1}\right\rangle=$ $\left\langle\nabla_{X_{2}} \nabla_{X_{1}} X_{2}, X_{1}\right\rangle$. Para calcular $\left\langle\nabla_{X_{2}} \nabla_{X_{1}} X_{2}, X_{1}\right\rangle$ derivamos a primeira igualdade de (3.15) em relação a $X_{2}$ e utilizamos as segundas igualdades de (3.18) e (3.19), obtendo assim,

$$
-\left\langle\nabla_{X_{2}} \nabla_{X_{1}} X_{2}, X_{1}\right\rangle=\frac{-c \lambda_{2}\left(\nu^{2}-b_{2}^{2}\right)}{\lambda_{2}-\lambda_{1}}-\frac{\nu^{2} b_{1}^{2}}{\left(\lambda_{2}-\lambda_{1}\right)^{2}} .
$$

Observe, pelas equações (3.17), que $\left\langle\nabla_{X_{2}} X_{2}, \nabla_{X_{1}} X_{1}\right\rangle=0$ e portanto $X_{1}\left\langle\nabla_{X_{2}} X_{2}, X_{1}\right\rangle=$ $\left\langle\nabla_{X_{1}} \nabla_{X_{2}} X_{2}, X_{1}\right\rangle$. Para calcular $\left\langle\nabla_{X_{1}} \nabla_{X_{2}} X_{2}, X_{1}\right\rangle$ derivamos a segunda igualdade de (3.17) em relação a $X_{1}$ e utilizamos a primeira equação de (3.18) e (3.19), obtendo assim,

$$
\left\langle\nabla_{X_{1}} \nabla_{X_{2}} X_{2}, X_{1}\right\rangle=\frac{c \lambda_{1}\left(\nu^{2}-b_{1}^{2}\right)}{\lambda_{2}-\lambda_{1}}-\frac{\nu^{2} b_{2}^{2}}{\left(\lambda_{2}-\lambda_{1}\right)^{2}} .
$$


Somando as equações $(3.23)$ e $(3.22)$, obtemos

$$
\left\langle\nabla_{X_{1}} \nabla_{X_{2}} X_{2}-\nabla_{X_{2}} \nabla_{X_{1}} X_{2}, X_{1}\right\rangle=-c \nu^{2}+\frac{c\left(\lambda_{2} b_{2}^{2}-\lambda_{1} b_{1}^{2}\right)}{\lambda_{2}-\lambda_{1}}-\frac{\nu^{2}\left(b_{1}^{2}+b_{2}^{2}\right)}{\left(\lambda_{2}-\lambda_{1}\right)^{2}}
$$

Então, pelas equações (3.24) e (3.21), concluimos que

$$
\lambda_{1} \lambda_{2}+2 c \nu^{2}+\frac{c\left(\lambda_{1} b_{1}^{2}-\lambda_{2} b_{2}^{2}\right)}{\lambda_{2}-\lambda_{1}}+\frac{2 \nu^{2}\left(b_{1}^{2}+b_{2}^{2}\right)}{\left(\lambda_{2}-\lambda_{1}\right)^{2}}=0 .
$$

O próximo resultado mostra que uma superfície mínima de $\mathbb{Q}_{c}^{2} \times \mathbb{R}$ que possui curvaturas principais constantes é totalmente geodésica.

Corolário 3.3.2. As superfícies mínimas de $\mathbb{Q}_{c}^{2} \times \mathbb{R}$ com curvaturas principais constantes são totalmente geodésicas.

Demonstração. Suponha que existe uma superfície mínima com duas curvaturas principais constantes e distintas $\lambda_{2}=-\lambda_{1}$. Então, pela Proposição 3.3.1, temos que

$$
-\lambda_{1}^{2}+2 c \nu^{2}-\frac{c \lambda_{1}\left(b_{1}^{2}+b_{2}^{2}\right)}{2 \lambda_{1}}+\frac{2 \nu^{2}\left(b_{1}^{2}+b_{2}^{2}\right)}{4 \lambda_{1}^{2}}=0 .
$$

Vimos que $\nu^{2}+b_{1}^{2}+b_{2}^{2}=1$ e portanto

$$
-\lambda_{1}^{2}+2 c \nu^{2}+\frac{c\left(\nu^{2}-1\right)}{2}+\frac{2 \nu^{2}\left(1-\nu^{2}\right)}{4 \lambda_{1}^{2}}=0
$$

ou seja,

$$
\nu^{4}-\nu^{2}\left(1+5 c \lambda_{1}^{2}\right)+\lambda_{1}^{2}\left(2 \lambda_{1}^{2}+c\right)=0 .
$$

Assim, obtemos uma equação biquadrada na variável $\nu$, com coeficientes constantes reais. Suponha que a equação (3.25) tenha solução. Então a função $\nu$ é constante e consequentemente $0=X_{i}(\nu)=-\left\langle A X_{i}, T\right\rangle=-b_{i} \lambda_{i}$, com $i \in\{1,2\}$. Se $\lambda_{1}=0$ então $\lambda_{2}=-\lambda_{1}=0$, o que não pode ocorrer já que estamos supondo $\lambda_{1} \neq \lambda_{2}$. Assim, $b_{1}=b_{2}=0$, o que implica que $T=0$. Logo, não existe superfície mínima de $\mathbb{Q}_{c}^{2} \times \mathbb{R}$ com duas curvaturas principais constantes e distintas.

A proposição seguinte mostra que uma hipersuperfície em $\mathbb{Q}_{c}^{n} \times \mathbb{R}, n \geq 4$ e $\nu \neq 0$, com três curvaturas principais constantes e distintas com multiplicidades constantes não pode ter duas curvaturas principais com multiplicidades iguais a 1. 
Proposição 3.3.3. Seja $f: M^{n} \rightarrow \mathbb{Q}_{c}^{n} \times \mathbb{R}, n \geq 4$, uma hipersuperfície com três curvaturas principais constantes e distintas, $\lambda, \mu$ e $\gamma$, de multiplicidades constantes. Suponha que $\nu(p) \neq 0$, para todo $p \in M^{n}$, então não podem existir duas curvaturas principais com multiplicidades iguais a 1 .

Demonstração. Suponha que existem duas curvaturas principais $\lambda$ e $\mu$ de multiplicidades iguais a 1 . Seja $\left\{X_{1}, X_{2}, X_{3}, \ldots, X_{n}\right\}$ um referencial de direções principais ortonormais tais que $A X_{1}=\lambda X_{1}, A X_{2}=\mu X_{2}$ e $A X_{j}=\gamma X_{j}$, para $j \geq 3$. Pela Observação 3.2.2, temos $T=b_{1} X_{1}+b_{2} X_{2}$, onde $b_{1}, b_{2}: U \rightarrow \mathbb{R}, U \subset M^{n}$, são funções diferenciáveis.

Pelas equações de Codazzi, dadas por (1.4), temos

$$
\begin{aligned}
\nabla_{X_{1}} A X_{2}-\nabla_{X_{2}} A X_{1}-A\left[X_{1}, X_{2}\right] & =c \nu\left(b_{2} X_{1}-b_{1} X_{2}\right) \\
\nabla_{X_{1}} A X_{j}-\nabla_{X_{j}} A X_{1}-A\left[X_{1}, X_{j}\right] & =-c \nu b_{1} X_{j}, \text { para cada } j \in\{3, \ldots, n\} \\
\nabla_{X_{2}} A X_{j}-\nabla_{X_{j}} A X_{2}-A\left[X_{2}, X_{j}\right] & =-c \nu b_{2} X_{j}, \text { para cada } j \in\{3, \ldots, n\} \\
\nabla_{X_{\beta}} A X_{j}-\nabla_{X_{j}} A X_{\beta}-A\left[X_{\beta}, X_{j}\right] & =0, \text { para cada } j \in\{3, \ldots, n\} \text { e } \beta \neq 1,2, j
\end{aligned}
$$

Pela equação (3.26), obtemos

$$
\mu \nabla_{X_{1}} X_{2}-\lambda \nabla_{X_{2}} X_{1}-A\left(\nabla_{X_{1}} X_{2}-\nabla_{X_{2}} X_{1}\right)=c \nu\left(b_{2} X_{1}-b_{1} X_{2}\right)
$$

ou seja,

$$
\sum_{k=1}^{n}\left\langle\nabla_{X_{1}} X_{2}, X_{k}\right\rangle(\mu I-A) X_{k}+\sum_{l=1}^{n}\left\langle\nabla_{X_{2}} X_{1}, X_{l}\right\rangle(A-\lambda I) X_{l}-c \nu b_{2} X_{1}+c \nu b_{1} X_{2}=0
$$

Assim,

$$
\begin{gathered}
\left\langle\nabla_{X_{1}} X_{2}, X_{1}\right\rangle(\mu-\lambda)-c \nu b_{2}=0 \\
\left\langle\nabla_{X_{2}} X_{1}, X_{2}\right\rangle(\mu-\lambda)+c \nu b_{1}=0 \\
\text { e }\left\langle\nabla_{X_{1}} X_{2}, X_{j}\right\rangle(\mu-\gamma)+\left\langle\nabla_{X_{2}} X_{1}, X_{j}\right\rangle(\gamma-\lambda)=0, \text { para cada } j \in\{3, \ldots, n\} .
\end{gathered}
$$

Procedemos de maneira análoga com as equações (3.27), (3.28) e (3.29). Pela equação (3.27), obtemos, para cada $j \in\{3, \ldots, n\}$,

$$
\begin{aligned}
\left\langle\nabla_{X_{1}} X_{j}, X_{1}\right\rangle(\gamma-\lambda) & =0 \\
\left\langle\nabla_{X_{j}} X_{1}, X_{j}\right\rangle(\gamma-\lambda)+c \nu b_{1} & =0 \\
\left\langle\nabla_{X_{j}} X_{1}, X_{\beta}\right\rangle(\gamma-\lambda) & =0, \quad \beta \neq 1,2, j, \quad \text { e } \\
\left\langle\nabla_{X_{1}} X_{j}, X_{2}\right\rangle(\gamma-\mu)+\left\langle\nabla_{X_{j}} X_{1}, X_{2}\right\rangle(\mu-\lambda) & =0 .
\end{aligned}
$$


Pela equação (3.28), concluimos, para cada $j \in\{3, \ldots, n\}$, que

$$
\begin{aligned}
\left\langle\nabla_{X_{2}} X_{j}, X_{2}\right\rangle(\gamma-\mu) & =0 \\
\left\langle\nabla_{X_{j}} X_{2}, X_{j}\right\rangle(\gamma-\mu)+c \nu b_{2} & =0 \\
\left\langle\nabla_{X_{j}} X_{2}, X_{\beta}\right\rangle(\gamma-\mu) & =0, \quad \beta \neq 1,2, j, \quad \text { e } \\
\left\langle\nabla_{X_{2}} X_{j}, X_{1}\right\rangle(\gamma-\lambda)+\left\langle\nabla_{X_{j}} X_{2}, X_{1}\right\rangle(\lambda-\mu) & =0 .
\end{aligned}
$$

Por (3.29), obtemos, para cada $j \in\{3, \ldots, n\}$ e $\beta \neq 1,2, j$,

$$
\begin{aligned}
& \left\langle\nabla_{X_{\beta}} X_{j}, X_{1}\right\rangle-\left\langle\nabla_{X_{j}} X_{\beta}, X_{1}\right\rangle=0 \\
& \left\langle\nabla_{X_{\beta}} X_{j}, X_{2}\right\rangle-\left\langle\nabla_{X_{j}} X_{\beta}, X_{2}\right\rangle=0
\end{aligned}
$$

Então, por (3.34), (3.38) e (3.40),

$$
\left\langle\nabla_{X_{\beta}} X_{j}, X_{1}\right\rangle=0 \quad \text { e } \quad\left\langle\nabla_{X_{\beta}} X_{j}, X_{2}\right\rangle=0
$$

Como consequência das equações de (3.30) até (3.39) e por (3.41), concluimos, para cada $j \in\{3, \ldots, n\}$, que

$$
\begin{aligned}
\nabla_{X_{1}} X_{1} & =-\frac{c \nu b_{2}}{\mu-\lambda} X_{2}, \\
\nabla_{X_{1}} X_{2} & =\frac{c \nu b_{2}}{\mu-\lambda} X_{1}+\sum_{j=3}^{n}\left\langle\nabla_{X_{2}} X_{1}, X_{j}\right\rangle \frac{(\lambda-\gamma)}{\mu-\gamma} X_{j}, \\
\nabla_{X_{1}} X_{j} & =\left\langle\nabla_{X_{j}} X_{1}, X_{2}\right\rangle \frac{(\lambda-\mu)}{\gamma-\mu} X_{2}+\sum_{\beta \neq 1,2, j}\left\langle\nabla_{X_{1}} X_{j}, X_{\beta}\right\rangle X_{\beta}, \\
\nabla_{X_{2}} X_{1} & =-\frac{c \nu b_{1}}{\mu-\lambda} X_{2}+\sum_{j=3}^{n}\left\langle\nabla_{X_{2}} X_{1}, X_{j}\right\rangle X_{j}, \\
\nabla_{X_{2}} X_{2} & =\frac{c \nu b_{1}}{\mu-\lambda} X_{1}, \\
\nabla_{X_{2}} X_{j} & =\left\langle\nabla_{X_{j}} X_{2}, X_{1}\right\rangle \frac{(\mu-\lambda)}{\gamma-\lambda} X_{1}+\sum_{\beta \neq 1,2, j}\left\langle\nabla_{X_{2}} X_{j}, X_{\beta}\right\rangle X_{\beta}, \\
\nabla_{X_{j}} X_{1} & =\left\langle\nabla_{X_{j}} X_{1}, X_{2}\right\rangle X_{2}-\frac{c \nu b_{1}}{\gamma-\lambda} X_{j}, \\
\nabla_{X_{j}} X_{2} & =\left\langle\nabla_{X_{j}} X_{2}, X_{1}\right\rangle X_{1}-\frac{c \nu b_{2}}{\gamma-\mu} X_{j},
\end{aligned}
$$




$$
\begin{aligned}
\nabla_{X_{j}} X_{j} & =\frac{c \nu b_{1}}{\gamma-\lambda} X_{1}+\frac{c \nu b_{2}}{\gamma-\mu} X_{2}+\sum_{\beta \neq 1,2, j}\left\langle\nabla_{X_{j}} X_{j}, X_{\beta}\right\rangle X_{\beta} \\
\nabla_{X_{\beta}} X_{j} & =\left\langle\nabla_{X_{\beta}} X_{j}, X_{\beta}\right\rangle X_{\beta}+\sum_{l \neq 1,2, j, \beta}\left\langle\nabla_{X_{\beta}} X_{j}, X_{l}\right\rangle X_{l} .
\end{aligned}
$$

Utilizando as igualdades (1.5) e (1.6), para todo $X \in T M^{n}$, temos que

$$
\begin{aligned}
& X_{1}(\nu)=-\lambda b_{1}, \\
& X_{2}(\nu)=-\mu b_{2}, \\
& X_{j}(\nu)=0, \quad j \in\{3, \ldots, n\} .
\end{aligned}
$$

Além disso, de

$$
\nabla_{X_{1}}\left(b_{1} X_{1}+b_{2} X_{2}\right)=X_{1}\left(b_{1}\right) X_{1}+b_{1} \nabla_{X_{1}} X_{1}+X_{1}\left(b_{2}\right) X_{2}+b_{2} \nabla_{X_{1}} X_{2}=\nu \lambda X_{1}
$$

concluimos que

$$
\begin{aligned}
X_{1}\left(b_{1}\right) & =\nu \lambda-\frac{c \nu b_{2}^{2}}{\mu-\lambda}, \\
X_{1}\left(b_{2}\right) & =\frac{c \nu b_{1} b_{2}}{\mu-\lambda}, \\
b_{2}\left\langle\nabla_{X_{1}} X_{2}, X_{j}\right\rangle & =0, \text { para cada } j \in\{3, \ldots, n\} .
\end{aligned}
$$

Analogamente, derivando $T$ em relação a $X_{2}$, temos

$$
\nabla_{X_{2}}\left(b_{1} X_{1}+b_{2} X_{2}\right)=X_{2}\left(b_{1}\right) X_{1}+b_{1} \nabla_{X_{2}} X_{1}+X_{2}\left(b_{2}\right) X_{2}+b_{2} \nabla_{X_{2}} X_{2}=\nu \mu X_{2}
$$

e portanto,

$$
\begin{aligned}
X_{2}\left(b_{1}\right) & =-\frac{c \nu b_{1} b_{2}}{\mu-\lambda} \\
X_{2}\left(b_{2}\right) & =\nu \mu+\frac{c \nu b_{1}^{2}}{\mu-\lambda}, \\
b_{1}\left\langle\nabla_{X_{2}} X_{1}, X_{j}\right\rangle & =0, \quad \text { para cada } j \in\{3, \ldots, n\} .
\end{aligned}
$$

Por um lado, pela equação de Gauss (1.3), temos

$$
\begin{aligned}
& K\left(X_{1}, X_{2}\right)=\lambda \mu+c \nu^{2}, \\
& K\left(X_{1}, X_{j}\right)=\lambda \gamma+c\left(1-b_{1}^{2}\right), \quad j \in\{3, \ldots, n\}, \\
& K\left(X_{2}, X_{j}\right)=\mu \gamma+c\left(1-b_{2}^{2}\right), \quad j \in\{3, \ldots, n\} .
\end{aligned}
$$


Por outro lado, pela definição de curvatura seccional, sabemos que

$$
K\left(X_{1}, X_{2}\right)=\left\langle R\left(X_{1}, X_{2}\right) X_{2}, X_{1}\right\rangle=\left\langle\nabla_{X_{1}} \nabla_{X_{2}} X_{2}-\nabla_{X_{2}} \nabla_{X_{1}} X_{2}-\nabla_{\left[X_{1}, X_{2}\right]} X_{2}, X_{1}\right\rangle
$$

Pelas equações (3.42) e (3.46), obtemos

$$
\left\langle\nabla_{X_{1}} \nabla_{X_{2}} X_{2}, X_{1}\right\rangle=X_{1}\left(\frac{c \nu b_{1}}{\mu-\lambda}\right)
$$

e então, por (3.52) e (3.53),

$$
\left\langle\nabla_{X_{1}} \nabla_{X_{2}} X_{2}, X_{1}\right\rangle=\frac{c \lambda\left(\nu^{2}-b_{1}^{2}\right)}{\mu-\lambda}-\frac{\nu^{2} b_{2}^{2}}{(\mu-\lambda)^{2}}
$$

Utilizando (3.43), (3.45), (3.52) e (3.57),

$$
\begin{aligned}
-\left\langle\nabla_{X_{2}} \nabla_{X_{1}} X_{2}, X_{1}\right\rangle & =\left\langle\nabla_{X_{1}} X_{2}, \nabla_{X_{2}} X_{1}\right\rangle-X_{2}\left\langle\nabla_{X_{1}} X_{2}, X_{1}\right\rangle \\
& =\sum_{j=3}^{n}\left\langle\nabla_{X_{2}} X_{1}, X_{j}\right\rangle^{2} \frac{(\lambda-\gamma)}{\mu-\gamma}-X_{2}\left(\frac{c \nu b_{2}}{\mu-\lambda}\right) \\
& =\sum_{j=3}^{n}\left\langle\nabla_{X_{2}} X_{1}, X_{j}\right\rangle^{2} \frac{(\lambda-\gamma)}{\mu-\gamma}-\frac{c \mu\left(\nu^{2}-b_{2}^{2}\right)}{\mu-\lambda}-\frac{\nu^{2} b_{1}^{2}}{(\mu-\lambda)^{2}}
\end{aligned}
$$

Observe que

$$
\begin{aligned}
\nabla_{\left[X_{1}, X_{2}\right]} X_{2}= & \frac{c \nu b_{2}}{\mu-\lambda} \nabla_{X_{1}} X_{2}+\sum_{j=3}^{n}\left\langle\nabla_{X_{2}} X_{1}, X_{j}\right\rangle \frac{(\lambda-\gamma)}{\mu-\gamma} \nabla_{X_{j}} X_{2}+ \\
& +\frac{c \nu b_{1}}{\mu-\lambda} \nabla_{X_{2}} X_{2}-\sum_{j=3}^{n}\left\langle\nabla_{X_{2}} X_{1}, X_{j}\right\rangle \nabla_{X_{j}} X_{2} .
\end{aligned}
$$

Assim,

$$
-\left\langle\nabla_{\left[X_{1}, X_{2}\right]} X_{2}, X_{1}\right\rangle=-\frac{\nu^{2}\left(b_{1}^{2}+b_{2}^{2}\right)}{(\mu-\lambda)^{2}}+\sum_{j=3}^{n}\left\langle\nabla_{X_{2}} X_{1}, X_{j}\right\rangle\left\langle\nabla_{X_{j}} X_{2}, X_{1}\right\rangle\left(1-\frac{(\lambda-\gamma)}{\mu-\gamma}\right)
$$

Por (3.47) e (3.49), temos que

$$
\left\langle\nabla_{X_{j}} X_{2}, X_{1}\right\rangle=\left\langle\nabla_{X_{2}} X_{j}, X_{1}\right\rangle \frac{(\gamma-\lambda)}{\mu-\lambda}
$$


e então

$$
-\left\langle\nabla_{\left[X_{1}, X_{2}\right]} X_{2}, X_{1}\right\rangle=-\frac{\nu^{2}\left(b_{1}^{2}+b_{2}^{2}\right)}{(\mu-\lambda)^{2}}-\sum_{j=3}^{n}\left\langle\nabla_{X_{2}} X_{j}, X_{1}\right\rangle^{2} \frac{(\gamma-\lambda)}{\mu-\lambda}\left(1-\frac{(\lambda-\gamma)}{\mu-\gamma}\right) .
$$

Logo, somando (3.62) com (3.63) e (3.64) e igualando a (3.59), obtemos

$$
\frac{2 \nu^{2}\left(1-\nu^{2}\right)}{(\mu-\lambda)^{2}}+2 c \nu^{2}+\lambda \mu+\frac{c\left(b_{1}^{2} \lambda-b_{2}^{2} \mu\right)}{\mu-\lambda}-\frac{2(\lambda-\gamma)}{\mu-\gamma} \sum_{j=3}^{n}\left\langle\nabla_{X_{2}} X_{1}, X_{j}\right\rangle^{2}=0
$$

Procedendo de maneira análoga para $K\left(X_{1}, X_{j}\right)$ e $K\left(X_{2}, X_{j}\right)$ concluimos, para cada $j \geq 3$,

$$
\begin{aligned}
& \frac{c \lambda\left(\nu^{2}-b_{1}^{2}\right)}{\gamma-\lambda}-\frac{\nu^{2}}{\gamma-\lambda}\left(\frac{b_{1}^{2}}{\gamma-\lambda}+\frac{b_{2}^{2}}{\mu-\lambda}\right)+\frac{\nu^{2} b_{2}^{2}}{(\mu-\lambda)(\gamma-\mu)}-\lambda \gamma-c\left(1-b_{1}^{2}\right)+ \\
& -\frac{2(\mu-\lambda)}{\gamma-\mu}\left\langle\nabla_{X_{j}} X_{1}, X_{2}\right\rangle^{2}=0 \\
& \frac{c \mu\left(\nu^{2}-b_{2}^{2}\right)}{\gamma-\mu}+\frac{\nu^{2}}{\gamma-\mu}\left(\frac{b_{1}^{2}}{\mu-\lambda}-\frac{b_{2}^{2}}{\gamma-\mu}\right)-\frac{\nu^{2} b_{1}^{2}}{(\gamma-\lambda)(\mu-\lambda)}-\mu \gamma-c\left(1-b_{2}^{2}\right)+ \\
& +\frac{2(\mu-\lambda)}{\gamma-\lambda}\left\langle\nabla_{X_{j}} X_{1}, X_{2}\right\rangle^{2}=0 .
\end{aligned}
$$

Por (3.58), temos $b_{1}\left\langle\nabla_{X_{2}} X_{1}, X_{j}\right\rangle=0$, para cada $j \in\{3, \ldots, n\}$. Suponha que $b_{1}(p)=0$, para todo $p \in U \subset M^{n}$. Então $T$ é uma direção principal. Como estamos supondo $\nu \neq 0$, pelo Teorema 3.1.2, temos que $c=-1$ e $g=2$, o que é um absurdo pois, por hipótese, $g=3$. Assim, deve existir um $p_{0}$ tal que $b_{1}\left(p_{0}\right) \neq 0$. Então, por continuidade da função $b_{1}$, existe uma vizinhança $V \subset U \subset M^{n}$ de $p_{0}$ tal que $b_{1}(p) \neq 0$, para todo $p \in V$. Logo, $\left\langle\nabla_{X_{2}} X_{1}, X_{j}\right\rangle=0$, em $V$, para cada $j \in\{3, \ldots, n\}$.

Pelas equações (3.31) e (3.35) concluimos que

$$
\left\langle\nabla_{X_{2}} X_{1}, X_{j}\right\rangle=0 \Leftrightarrow\left\langle\nabla_{X_{1}} X_{2}, X_{j}\right\rangle=0 \Leftrightarrow\left\langle\nabla_{X_{j}} X_{1}, X_{2}\right\rangle=0, \quad \text { para cada } j \in\{3, \ldots, n\} \text {. }
$$

E portanto, pelas equações (3.65), (3.66) e (3.67), obtemos em $V$, respectivamente,

$$
\begin{gathered}
\frac{2 \nu^{2}\left(1-\nu^{2}\right)}{(\mu-\lambda)^{2}}+2 c \nu^{2}+\lambda \mu+\frac{c\left(b_{1}^{2} \lambda-b_{2}^{2} \mu\right)}{\mu-\lambda}=0, \\
\frac{c \lambda\left(\nu^{2}-b_{1}^{2}\right)}{\gamma-\lambda}-\frac{\nu^{2} b_{1}^{2}}{(\gamma-\lambda)^{2}}+\frac{\nu^{2} b_{2}^{2}}{(\gamma-\mu)(\gamma-\lambda)}-\lambda \gamma-c\left(1-b_{1}^{2}\right)=0,
\end{gathered}
$$




$$
\frac{c \mu\left(\nu^{2}-b_{2}^{2}\right)}{\gamma-\mu}-\frac{\nu^{2} b_{2}^{2}}{(\gamma-\mu)^{2}}+\frac{\nu^{2} b_{1}^{2}}{(\gamma-\mu)(\gamma-\lambda)}-\mu \gamma-c\left(1-b_{2}^{2}\right)=0 .
$$

Substituindo $b_{2}^{2}$ por $1-\nu^{2}-b_{1}^{2}$ na equação (3.68), obtemos

$$
-c b_{1}^{2} \frac{(\lambda+\mu)}{\mu-\lambda}=\lambda \mu+2 c \nu^{2}-\frac{c \mu\left(1-\nu^{2}\right)}{\mu-\lambda}+\frac{2 \nu^{2}\left(1-\nu^{2}\right)}{(\mu-\lambda)^{2}} .
$$

Com argumentos análogos aos do Corolário 3.3.2, concluimos que $\lambda+\mu \neq 0$. Então

$$
b_{1}^{2}=\nu^{4} \frac{2 c}{(\mu+\lambda)(\mu-\lambda)}+\nu^{2} \frac{(\mu-\lambda)(-3 \mu+2 \lambda)-2 c}{(\mu+\lambda)(\mu-\lambda)}+\frac{\mu}{\mu+\lambda}(1-c \lambda(\mu-\lambda)) \text {. }
$$

Somando (3.69) com (3.70) temos

$$
\begin{aligned}
& c \nu^{2}\left(\frac{\lambda}{\gamma-\lambda}+\frac{\mu}{\gamma-\mu}\right)-\frac{c \lambda b_{1}^{2}}{\gamma-\lambda}-\frac{c \mu b_{2}^{2}}{\gamma-\mu}-\frac{\nu^{2} b_{1}^{2}}{(\gamma-\lambda)^{2}}-\frac{\nu^{2} b_{2}^{2}}{(\gamma-\mu)^{2}}+ \\
& +\frac{\nu^{2}\left(1-\nu^{2}\right)}{(\gamma-\mu)(\gamma-\lambda)}-\gamma(\mu+\lambda)-c\left(1+\nu^{2}\right)=0 .
\end{aligned}
$$

Substituindo em (3.72) $b_{2}^{2}$ por $1-\nu^{2}-b_{1}^{2}$ obtemos

$$
\begin{aligned}
& \nu^{4} \frac{(\mu-\lambda)}{(\gamma-\mu)^{2}(\gamma-\lambda)}+\nu^{2}\left(\frac{-(\mu-\lambda)+c \lambda(\gamma-\mu)^{2}+2 c \mu(\gamma-\mu)(\gamma-\lambda)}{(\gamma-\mu)^{2}(\gamma-\lambda)}-c\right)-\frac{c \gamma}{\gamma-\mu}+ \\
& -\gamma(\mu+\lambda)+c b_{1}^{2}\left(\frac{\mu}{\gamma-\mu}-\frac{\lambda}{\gamma-\lambda}\right)+\nu^{2} b_{1}^{2}\left(\frac{1}{(\gamma-\mu)^{2}}-\frac{1}{(\gamma-\lambda)^{2}}\right)=0 .
\end{aligned}
$$

Substituindo (3.71) em (3.73) observamos que a maior potência de $\nu$ nessa última equação é $\nu^{6}$, que tem por coeficiente $\left(\frac{1}{(\gamma-\mu)^{2}}-\frac{1}{(\gamma-\lambda)^{2}}\right) \frac{2 c}{(\mu+\lambda)(\mu-\lambda)}$. Se esse termo não for nulo, a equação será de grau 6 com coeficientes constantes reais, onde a variável é a função $\nu$. Caso contrário, isto é, se $(\gamma-\lambda)^{2}=(\gamma-\mu)^{2}$ temos $\gamma-\lambda=\gamma-\mu$ ou $\gamma-\lambda=-(\gamma-\mu)$. Se $\gamma-\lambda=\gamma-\mu$ então $\lambda=\mu$, o que contradiz a hipótese de $\lambda \neq \mu$. Se $\gamma-\lambda=-(\gamma-\mu)$ então $\mu-\lambda=2(\gamma-\lambda)$. Assim, a maior potência de $\nu$ será de ordem 4 e o coeficiente de $\nu^{4}$ é $\frac{1}{(\gamma-\mu)^{2}}$, que é não nulo. Logo, nesse caso, teríamos uma equação biquadrada de coeficientes constantes reais na variável $\nu$.

Logo, supondo a existência de duas curvaturas principais constantes de multiplicidade 1 e $\nu(p) \neq 0$, para todo $p \in M^{n}$, temos que a função $\nu$ deve satisfazer essa equação. Dessa forma, a função $\nu$ será constante e portanto o campo $T$ é uma direção principal. Mas então, pelo Teorema 3.1.2, temos $c=-1$ e $g=2$, o que é um absurdo, pois estamos supondo $g=3$. 
Concluimos assim que não podem existir duas curvaturas principais constantes de multiplicidade 1 , supondo $\nu(p) \neq 0$, para todo $p \in M^{n}$.

Com os resultados anteriores, estamos preparados para provar o seguinte teorema de classificação local das hipersuperfícies em $\mathbb{Q}_{c}^{n} \times \mathbb{R}, n \neq 3$, com curvaturas principais constantes e $g \in\{1,2,3\}$.

Teorema 3.3.4. Seja $f: M^{n} \rightarrow \mathbb{Q}_{c}^{n} \times \mathbb{R}$ uma hipersuperfície com curvaturas principais constantes.

(i) Se $g=1$ e $n \geq 2$ então $f\left(M^{n}\right)$ é um subconjunto aberto de $\mathbb{Q}_{c}^{n} \times\left\{t_{0}\right\}$, para algum $t_{0} \in \mathbb{R}$, ou de um produto Riemanniano $M^{n-1} \times \mathbb{R}$. Nesse último caso, para $c=1, M^{n-1}$ é uma esfera totalmente geodésica em $\mathbb{S}^{n}$ e, para $c=-1, M^{n-1}$ é um hiperplano totalmente geodésico em $\mathbb{H}^{n}$.

(ii) Se $g=2$ e $n \geq 2$ então $c=-1$ e $f$ é localmente dada por $f(x, s)=h_{s}(x)+B s \partial / \partial t$, para algum $B \in \mathbb{R}, B>0$, com $M^{n}=M^{n-1} \times I$, onde $h_{s}$ é uma família de horosferas em $\mathbb{H}^{n}$, ou $f\left(M^{n}\right)$ é um subconjunto aberto de um produto Riemanniano $M^{n-1} \times \mathbb{R}$. Nesse último caso, se c $=1$ então $M^{n-1}$ é uma esfera não totalmente geodésica em $\mathbb{S}^{n}$ e, se $c=-1, M^{n-1}$ é uma hipersuperfície equidistante, uma horosfera ou uma hiperesfera $e m \mathbb{H}^{n}$.

(iii) Se $g=3, n \geq 4$ e as multiplicidades das curvaturas principais são constantes, então para $c=1, f\left(M^{n}\right)$ é um subconjunto aberto de um produto Riemanniano $\mathbb{S}^{p}(r) \times \mathbb{S}^{q}(s) \times \mathbb{R}$, com $n=p+q+1$ e $r^{2}+s^{2}=1$, ou do produto $M^{n-1} \times \mathbb{R}$, onde $M^{n-1}$ é uma hipersuperfície de Cartan para $n \in\{4,7,13,25\}$. Se $c=-1, f\left(M^{n}\right)$ é um aberto do produto Riemanniano $\mathbb{S}^{k} \times \mathbb{H}^{n-k-1} \times \mathbb{R}$.

Demonstração. (i) Suponha que $g=1$. Se $\nu \equiv 0$ então o campo $T$ é uma direção principal com curvatura principal correspondente $\lambda=0$, pois $X(\nu)=-\langle A X, T\rangle$, para todo $X \in T M$. Assim, $f$ umbílica implica que todas as curvaturas principais são nulas. Logo $f$ é totalmente geodésica.

Suponha agora que $\nu(p) \neq 0$, para todo $p \in M$. Seja $\left\{X_{1}, X_{2}, \ldots, X_{n}\right\}$ um referencial local ortonormal de $f$. Podemos escrever $T=\sum_{i=1}^{n} b_{i} X_{i}$. Por hipótese, temos que $A X_{i}=\lambda X_{i}$ para todo $i \in\{1, \ldots, n\}$, onde $\lambda$ é uma constante em $\mathbb{R}$.

Pela equação de Codazzi temos,

$$
\nabla_{X_{i}} A X_{j}-\nabla_{X_{j}} A X_{i}-A\left[X_{i}, X_{j}\right]=c \nu\left(b_{j} X_{i}-b_{i} X_{j}\right)
$$


o que implica em $0=\lambda\left[X_{i}, X_{j}\right]-\lambda\left[X_{i}, X_{j}\right]=c \nu\left(b_{j} X_{i}-b_{i} X_{j}\right)$. Uma vez que $\nu \neq 0$ e $X_{i}$ e $X_{j}$ são linearmente independentes para $i \neq j$, temos que ter $b_{i}=b_{j}=0$ para todo $i, j \in\{1, \ldots, n\}$. Assim, $T=0$ e portanto $f\left(M^{n}\right)$ é localmente um subconjunto aberto de uma fatia $\mathbb{Q}_{c}^{n} \times\{t\}$, o que prova o item (i).

(ii) Considere agora $g=2$. Observe que pela Proposição 1.2.1, as multiplicidades das curvaturas principais são constantes. Dividiremos esse caso em dois subcasos: $n=2$ e $n \geq 3$.

Caso 1: $n=2$

Fazendo $b_{2}^{2}=1-\nu^{2}-b_{1}^{2}$ na equação (3.13) obtemos,

$$
-c b_{1}^{2} \frac{\left(\lambda_{1}+\lambda_{2}\right)}{\lambda_{2}-\lambda_{1}}=\lambda_{1} \lambda_{2}+2 c \nu^{2}-\frac{c \lambda_{2}\left(1-\nu^{2}\right)}{\lambda_{2}-\lambda_{1}}+\frac{2 \nu^{2}\left(1-\nu^{2}\right)}{\left(\lambda_{2}-\lambda_{1}\right)^{2}} .
$$

Pelo Corolário 3.3.2, temos que $\lambda_{1}+\lambda_{2} \neq 0$ e assim,

$$
b_{1}^{2}=-c \frac{\left(\lambda_{2}-\lambda_{1}\right)}{\lambda_{1}+\lambda_{2}}\left\{\lambda_{1} \lambda_{2}+2 c \nu^{2}-\frac{c \lambda_{2}\left(1-\nu^{2}\right)}{\lambda_{2}-\lambda_{1}}+\frac{2 \nu^{2}\left(1-\nu^{2}\right)}{\left(\lambda_{2}-\lambda_{1}\right)^{2}}\right\} .
$$

Substituindo $b_{1}^{2}=1-\nu^{2}-b_{2}^{2}$ na equação (3.13) obtemos,

$$
-2 \nu^{4}+\nu^{2} D+b_{2}^{2} E=F
$$

onde, $D=2 c\left(\lambda_{2}-\lambda_{1}\right)^{2}-c \lambda_{1}\left(\lambda_{2}-\lambda_{1}\right)+2, E=-c\left(\lambda_{2}^{2}-\lambda_{1}^{2}\right)$ e $F=-\lambda_{1} \lambda_{2}\left(\lambda_{2}-\lambda_{1}\right)^{2}-c \lambda_{1}\left(\lambda_{2}-\lambda_{1}\right)$. Derivando a equação (3.75) em relação a $X_{2}$, temos que

$$
-8 \nu^{3} X_{2}(\nu)+2 \nu X_{2}(\nu) D+2 b_{2} X_{2}\left(b_{2}\right) E=0
$$

Por (3.18),

$$
X_{2}\left(b_{2}\right)=\nu \lambda_{2}+\frac{c \nu\left(b_{1}\right)^{2}}{\lambda_{2}-\lambda_{1}},
$$

e por (3.19), $X_{2}(\nu)=-\lambda_{2} b_{2}$. Então, se $\lambda_{2}=0$ temos $X_{2}(\nu)=0$ e pela equação (3.76), $b_{2} X_{2}\left(b_{2}\right)=0$ o que implica em $b_{2}=0$ ou, caso contrário, $\nu=0$ ou $b_{1}=0$. Se $\nu \equiv 0$, então $T$ é uma direção principal. Se $\nu \neq 0$, então $b_{2}=0$ ou $b_{1}=0$. Suponha que existe $x_{0}$ tal que $b_{1}\left(x_{0}\right) \neq 0$, então, por continuidade, existe uma vizinhança $V$ de $x_{0}$, tal que $b_{1}(x) \neq 0$, para todo $x \in V$. Assim, $b_{2}(x)=0$, para todo $x \in V$ e $T$ é uma direção principal. Ou seja, se $\lambda_{2}=0$, então $T$ é uma direção principal.

Suponha que $\lambda_{2} \neq 0$. Então $b_{2}=-\frac{X_{2}(\nu)}{\lambda_{2}}$ e pela equação (3.76) temos

$$
X_{2}(\nu)\left\{-8 \nu^{3}+2 \nu D-\frac{2 E}{\lambda_{2}} X_{2}\left(b_{2}\right)\right\}=0
$$


Logo, $X_{2}(\nu)=0$ ou $-8 \nu^{3}+2 \nu D-\frac{2 E}{\lambda_{2}} X_{2}\left(b_{2}\right)=0$.

Se $X_{2}(\nu)=0$ temos $b_{2}=0$ e portanto $T$ é uma direção principal. Caso contrário,

$$
-8 \nu^{3}+2 \nu D-\frac{2 E}{\lambda_{2}}\left(\nu \lambda_{2}+\frac{c \nu\left(b_{1}\right)^{2}}{\lambda_{2}-\lambda_{1}}\right)=0
$$

Então,

$$
-8 \nu^{3}+4 \nu+4 c \nu\left(\lambda_{2}-\lambda_{1}\right)^{2}-2 c \nu \lambda_{1}\left(\lambda_{2}-\lambda_{1}\right)+2 c \nu\left(\lambda_{2}^{2}-\lambda_{1}^{2}\right)+\frac{2 \nu\left(\lambda_{2}+\lambda_{1}\right)}{\lambda_{2}} b_{1}^{2}=0 .
$$

Por (3.74), temos que

$$
\frac{2 \nu\left(\lambda_{2}+\lambda_{1}\right)}{\lambda_{2}} b_{1}^{2}=-2 c \nu \lambda_{1}\left(\lambda_{2}-\lambda_{1}\right)+2 \nu\left(1-\nu^{2}\right)-\frac{4 \nu^{3}\left(\lambda_{2}-\lambda_{1}\right)}{\lambda_{2}}-\frac{4 c \nu^{3}\left(1-\nu^{2}\right)}{\lambda_{2}\left(\lambda_{2}-\lambda_{1}\right)} .
$$

Substituindo (3.78) em (3.77), obtemos

$$
\frac{4 c}{\lambda_{2}\left(\lambda_{2}-\lambda_{1}\right)} \nu^{5}-\nu^{3}\left(10+4 \frac{\left(\lambda_{2}-\lambda_{1}\right)^{2}+c}{\lambda_{2}\left(\lambda_{2}-\lambda_{1}\right)}\right)+6 \nu\left(1+c\left(\lambda_{2}-\lambda_{1}\right)^{2}\right)=0 .
$$

Observe que $\frac{4 c}{\lambda_{2}\left(\lambda_{2}-\lambda_{1}\right)} \neq 0$ e assim, obtemos uma equação de grau 5 onde a variável é a função $\nu$.

Note que $\nu \equiv 0$ é uma solução para essa equação e nesse caso, $T$ é uma direção principal. Se existirem outras soluções para essa equação, então a função $\nu$ é constante e portanto $T$ é uma direção principal.

Assim, concluímos para $g=2$ e $n=2$ que $T$ é uma direção principal. Analisaremos agora, o que ocorre quando $\nu \equiv 0$ e $\nu \neq 0$.

Se $\nu \neq 0$, então a superfície é localmente dada pelo Teorema 3.1.2. Se $\nu=0$ então a superfície é um cilindro sobre uma curva, $f\left(M^{2}\right)=\alpha \times \mathbb{R}$, onde $\alpha$ é um círculo não totalmente geodésico em $\mathbb{S}^{2}$, para $c=1$, ou $\alpha$ é uma curva equidistante, um horociclo ou um círculo hiperbólico em $\mathbb{H}^{2}$, para $c=-1$.

Caso 2: $n \geq 3$

Se $\nu(p) \neq 0$, para todo $p \in M$, pelo Teorema 3.2.1, $f$ possui pelo menos uma curvatura principal de multiplicidade 1 . Uma vez que $n \geq 3$ e $g=2$, existe uma única curvatura de multiplicidade 1 e pela Proposição 3.2.4, $T$ é uma direção principal correspondente a essa curvatura. Portanto $f$ é dada pelo Teorema 3.1.2. Observe que pela Proposição 1.1.10 essas hipersuperfícies são de rotação.

Se $\nu \equiv 0$ então $f\left(M^{n}\right)$ é um subconjunto aberto de um produto Riemanniano $M^{n-1} \times \mathbb{R}$, 
onde $M^{n-1}$ é uma hipersuperfície de $\mathbb{Q}_{c}^{n}$. Uma vez que a curvatura principal correspondente ao fator $\mathbb{R}$ é nula e pelo Teorema 1.2 .3 , e também no caso $c=1$ a outra curvatura não pode ser nula, temos que ter $M^{n-1}$ isoparamétrica umbílica não totalmente geodésica em $\mathbb{Q}_{c}^{n}$, o que prova o item (ii).

(iii) Suponha agora que $g=3$. Para ilustrar o caso $n \geq 4$, faremos uma tabela, para $n \in\{4,5,6,7,8\}$, com as possíveis multiplicidades das curvaturas principais de acordo com a dimensão $n$. No que segue, $m(\lambda), m(\mu)$ e $m(\gamma)$ denotarão as multiplicidade de $\lambda$, $\mu$ e $\gamma$, respectivamente.

\begin{tabular}{|c|c|c|c|}
\hline Dimensão & $m(\lambda)$ & $m(\mu)$ & $m(\gamma)$ \\
\hline \hline $\mathrm{n}=4$ & 1 & 1 & 2 \\
\hline \hline $\mathrm{n}=5$ & 1 & 1 & 3 \\
\cline { 2 - 4 } & 1 & 2 & 2 \\
\hline \hline \multirow{3}{*}{$\mathrm{n}=6$} & 1 & 1 & 4 \\
\cline { 2 - 4 } & 1 & 2 & 3 \\
\cline { 2 - 4 } & 2 & 2 & 2 \\
\hline \hline \multirow{3}{*}{$\mathrm{n}=7$} & 1 & 1 & 5 \\
\cline { 2 - 4 } & 1 & 2 & 4 \\
\cline { 2 - 4 } & 1 & 3 & 3 \\
\cline { 2 - 4 } & 2 & 2 & 3 \\
\hline \hline \multirow{3}{*}{$\mathrm{n}=8$} & 1 & 1 & 6 \\
\cline { 2 - 4 } & 1 & 2 & 5 \\
\cline { 2 - 4 } & 1 & 3 & 4 \\
\cline { 2 - 4 } & 2 & 2 & 4 \\
\cline { 2 - 4 } & 2 & 3 & 3 \\
\hline
\end{tabular}

Tabela 3.1: Possíveis multiplicidades para as curvaturas principais

De acordo com a dimensão considerada, podem existir três possibilidades para as multiplicidades das curvaturas principais: duas curvaturas possuem multiplicidade 1, apenas uma curvatura tem multiplicidade 1 ou todas as curvaturas possuem multiplicidades $\geq 2$. Vamos analisar cada um desses casos.

Caso 1) Suponha que duas curvaturas possuem multiplicidade 1.

Pela Proposição 3.3.3, não pode acontecer $\nu(p) \neq 0$, para todo $p \in M^{n}$. Portanto, $\nu \equiv 0$ e consequentemente, $f\left(M^{n}\right)$ é um subconjunto aberto de um produto Riemanniano $M^{n-1} \times \mathbb{R}$. Logo, uma das curvaturas é nula.

Para $c=-1, \lambda=0$ e as curvaturas principais de uma hipersuperfície isoparamétrica com $g=2 \mathrm{em} \mathbb{H}^{n}$ satisfazem $\mu \gamma=1$ e portanto, $\lambda=0$ tem multiplicidade 1 . Temos que $f\left(M^{n}\right)$ é localmente $\mathbb{S}^{1} \times \mathbb{H}^{n-2} \times \mathbb{R}$ ou $\mathbb{S}^{n-2} \times \mathbb{H}^{1} \times \mathbb{R}$. 
Para $c=1$ e $n \geq 4, f\left(M^{n}\right)$ é um subconjunto aberto $\mathbb{S}^{1} \times \mathbb{S}^{n-2} \times \mathbb{R}$. Além disso, para $n=4$, também pode ocorrer que $f\left(M^{n}\right)$ é localmente dada por $M^{3} \times \mathbb{R}$, onde $M^{3}$ é um tubo sobre uma Veronese em $\mathbb{S}^{4}$.

Caso 2) Suponha que apenas uma curvatura tem multiplicidade 1.

Se $\nu(p) \neq 0$, para todo $p \in M^{n}$, pela Proposição 3.2.4, o campo $T$ é uma direção principal e pelo Teorema 3.1.2, temos que $c=-1$ e $g=2$, o que não pode ocorrer. Portanto $\nu \equiv 0$ e $f\left(M^{n}\right)$ é um subconjunto aberto de um produto Riemanniano $M^{n-1} \times \mathbb{R}$. Nesse caso a curvatura que tem multiplicidade 1 é $\lambda=0$. A seguir, explicitamos $M^{n-1}$ nos casos $c=-1$ e $c=1$.

Para $c=-1$, temos que $f\left(M^{n}\right)$ é um subconjunto aberto de $\mathbb{S}^{k} \times \mathbb{H}^{n-k-1} \times \mathbb{R}$, com $k \geq 2$ e $n \geq 5$. Para $c=1, f\left(M^{n}\right)$ é um subconjunto aberto de $\mathbb{S}^{k} \times \mathbb{S}^{n-k-1} \times \mathbb{R}$, com $k \geq 2$ e $n \geq 5$.

Caso 3) Finalmente, suponha que todas as curvaturas possuem multiplicidades $\geq 2$.

Pela Observação 3.2.2, temos que $\nu \equiv 0$ e assim uma das curvaturas principais é $\lambda=0$. Além disso, $f\left(M^{n}\right)$ é um subconjunto aberto de um produto Riemanniano $M^{n-1} \times \mathbb{R}$.

Portanto, para $c=-1$, pelo Teorema 1.2.3, não existem hipersuperfícies isoparamétricas não totalmente geodésicas em $\mathbb{H}^{n}$ com uma curvatura principal nula. Nesse caso, $\lambda=0$ teria multiplicidade 1, o que não pode ocorrer.

Para $c=1$, as hipersuperfícies isoparamétricas em $\mathbb{S}^{n} \operatorname{com} g=3$, são as hipersuperfícies de Cartan. Essas hipersuperfícies possuem uma curvatura principal nula. Logo, esse caso ocorre para $n \in\{7,13,25\}$ e $f\left(M^{n}\right)$ é localmente $M^{n-1} \times \mathbb{R}$, onde $M^{n-1}$ é uma hipersuperfície de Cartan em $\mathbb{S}^{n}$.

Observação 3.3.5. As hipersuperfícies classificadas pelo Teorema 3.3.4 possuem função $\nu$ constante e pelo Corolário 2.3.6, elas são isoparamétricas em $\mathbb{Q}_{c}^{n} \times \mathbb{R}$. 


\section{Referências Bibliográficas}

[1] Abresch, U. Isoparametric hypersurfaces with four or six distinct principal curvatures. Necessary conditions on the multiplicities. Math. Ann. 264 (1983), 283-302. 18

[2] Abresch, U., and Rosenberg, H. A Hopf differential for constant mean curvature surfaces in $\mathbb{S}^{2} \times \mathbb{R}$ and $\mathbb{H}^{2} \times \mathbb{R}$. Acta Math. 193, 2 (2004), 141-174. xiii

[3] Aledo, J. A., Espinar, J. M., and Gálvez, J. A. Complete surfaces of constant curvature in $H^{2} \times \mathbb{R}$ and $S^{2} \times \mathbb{R}$. Calc. Var. Partial Differ. Equ. 29, 3 (2007), 347-363. xiii

[4] Aledo, J. A., Espinar, J. M., And Gálvez, J. A. Surfaces with constant curvature in $S^{2} \times \mathbb{R}$ and $H^{2} \times \mathbb{R}$. Height estimates and representation. Bull. Braz. Math. Soc. (N.S.) 38,4 (2007), 533-554. xiii

[5] Cartan, E. Familles de surfaces isoparamétriques dans les espaces à courbure constante. Ann. Mat. pura appl., Bologna, (4) 17 (1938), 177-191. xiv, 15

[6] Cartan, E. Sur des familles remarquables d'hypersurfaces isoparamétriques dans les espaces sphériques. Math. Z. 45 (1939), 335-367. xiv

[7] Cartan, E. Sur quelques familles remarquables d'hypersurfaces. C.R. Congrès Math. Liège, 1939, pp. 30-41. xiv, 15, 18

[8] Cartan, E. Sur des familles d'hypersurfaces isoparamétriques des espaces sphériques à 5 et à 9 dimensions. Rev. Univ. Nac. Tucuman, A 1, 5-22 (1940)., 1940. xiv

[9] Cecil, T., And Ryan, P. Tight and taut immersions of manifolds, vol. 107. Research Notes in Mathematics, Boston - London - Melbourne, 1985. 13, 15, 27

[10] Cecil, T. E., Chi, Q.-S., And Jensen, G. R. Isoparametric hypersurfaces with four principal curvatures. Ann. Math. (2) 166, 1 (2007), 1-76. 18 
[11] CHI, Q.-S. Isoparametric hypersurfaces with four principal curvatures. II. Nagoya Math. J. 204 (2011), 1-18. 18

[12] CHI, Q.-S. Isoparametric hypersurfaces with four principal curvatures. III. J. Differ. Geom. 94, 3 (2013), 469-504. 18

[13] Dajczer, M., Antonucci, M., Oliveira, G., Lima-Filho, P., and Tojeiro, R. Submanifolds and isometric immersions. Mathematics Lecture Series, 13. Houston, Texas: Publish or Perish, Inc. viii, 173 p. , 1990. 19

[14] Deszcz, R., and Yaprak, S. Curvature properties of Cartan hypersurfaces. Colloq. Math. 67, 1 (1994), 91-98. 18

[15] Dillen, F., Fastenakels, J., and Van Der Veken, J. Rotation hypersurfaces in $\mathbb{S}^{n} \times \mathbb{R}$ and $\mathbb{H}^{n} \times \mathbb{R}$. Note Mat. 29, 1 (2009), 41-54. xiii, 4, 5, 10

[16] Do Carmo, M. P., and Dajczer, M. Rotation hypersurfaces in spaces of constant curvature. Trans. Am. Math. Soc. 277 (1983), 685-709. xiii, 5, 8

[17] Espinar, J. M., Gálvez, J. A., And Rosenberg, H. Complete surfaces with positive extrinsic curvature in product spaces. Comment. Math. Helv. 84, 2 (2009), 351-386. xiii

[18] Ferus, D. Notes on isoparametric hypersurfaces. Escola de Geometria Diferencial, Universidade Estadual de Campinas, 1980. 15, 17

[19] Manfio, F., And Tojeiro, R. Hypersurfaces with constant sectional curvature of $\mathbb{S}^{n} \times \mathbb{R}$ and $\mathbb{H}^{n} \times \mathbb{R}$. Ill. J. Math. 55, 1 (2011), 397-415. xiv, 2, 10

[20] Meeks, W. H., and Rosenberg, H. Stable minimal surfaces in $M \times \mathbb{R}$. J. Differ. Geom. 68, 3 (2004), 515-534. xiii

[21] MiyaokA, R. Isoparametric hypersurfaces with $(g, m)=(6,2)$. Ann. Math. (2) 177, 1 (2013), 53-110. 18

[22] Montaldo, S., And Onnis, I. Enneper representation and the Gauss map of minimal surfaces in the product $\mathbb{H}^{2} \times \mathbb{R}$. Mat. Contemp. 33 (2007), 197-211. xiii

[23] Montaldo, S., and Onnis, I. I. Invariant CMC surfaces in $\mathbb{H}^{2} \times \mathbb{R}$. Glasg. Math. J. 46, 2 (2004), 311-321. xiii

[24] Münzner, H. F. Isoparametrische Hyperflächen in Sphären. I. Math. Ann. 251 (1980), 57-71. 18 
[25] MÜnZner, H. F. Isoparametrische Hyperflächen in Sphären. II: Über die Zerlegung der Sphäre in Ballbündel. Math. Ann. 256 (1981), 215-232. 18

[26] NeLLI, B. On properties of constant mean curvature surfaces in $\mathbb{H}^{2} \times \mathbb{R}^{1}$. In Geometry seminars. 2005-2009. Dedicated to Aldo Andreotti on the 13th anniversary of his passing. Bologna: Università degli Studi di Bologna, Dipartimento di Matematica, 2010, pp. 97104. xiii

[27] Nomizu, K. Characteristic roots and vectors of a differentiable family of symmetric matrices. Linear Multilinear Algebra 1 (1973), 159-162. 19, 20, 26

[28] OnNis, I. I. Invariant surfaces with constant mean curvature in $\mathbb{H}^{2} \times \mathbb{R}$. Ann. Mat. Pura Appl. (4) 187, 4 (2008), 667-682. xiii

[29] Rosenberg, H. Minimal surfaces in $M^{2} \times \mathbb{R}$. Ill. J. Math. 46, 4 (2002), 1177-1195. xiii

[30] Ryan, P. Homogeneity and some curvature conditions for hypersurfaces. Tohoku Math. J., II. Ser. 21 (1969), 363-388. 15

[31] Thorbergsson, G. A survey on isoparametric hypersurfaces and their generalizations. Amsterdam: North-Holland, 2000. xiv, 15

[32] Tojeiro, R. On a class of hypersurfaces in $\mathbb{S}^{n} \times \mathbb{R}$ and $\mathbb{H}^{n} \times \mathbb{R}$. Bull. Braz. Math. Soc. (N.S.) 41, 2 (2010), 199-209. xiii, 10, 11, 37

[33] Van der Veken, J., And VRancken, L. Parallel and semi-parallel hypersurfaces of $\mathbb{S}^{n} \times \mathbb{R}$. Bull. Braz. Math. Soc. (N.S.) 39, 3 (2008), 355-370. 4

[34] Wang, Q.-M. Isoparametric hypersurfaces in complex projective spaces. Differential geometry and differential equations, Proc. 1980 Beijing Sympos., Vol. 3, 1509-1523 (1982)., 1982. xiv

[35] YAu, S. T. Open problems in geometry. In Differential geometry. Part 1: Partial differential equations on manifolds. Proceedings of a summer research institute, held at the University of California, Los Angeles, CA, USA, July 8-28, 1990. Providence, RI: American Mathematical Society, 1993, pp. 1-28. 18 